*wals View/Frint Document Cover Sheet

This document was retrieved from the Boeing ISEARCH System.

Accession \#: D196050048

Document \#: SD-CP-TI-197

Title/Desc:

GENII DOSE CALCULATIONS FOR OFFSITE MXIMUM INDIVIDUAL \& POPULATIONS FROM PFP 


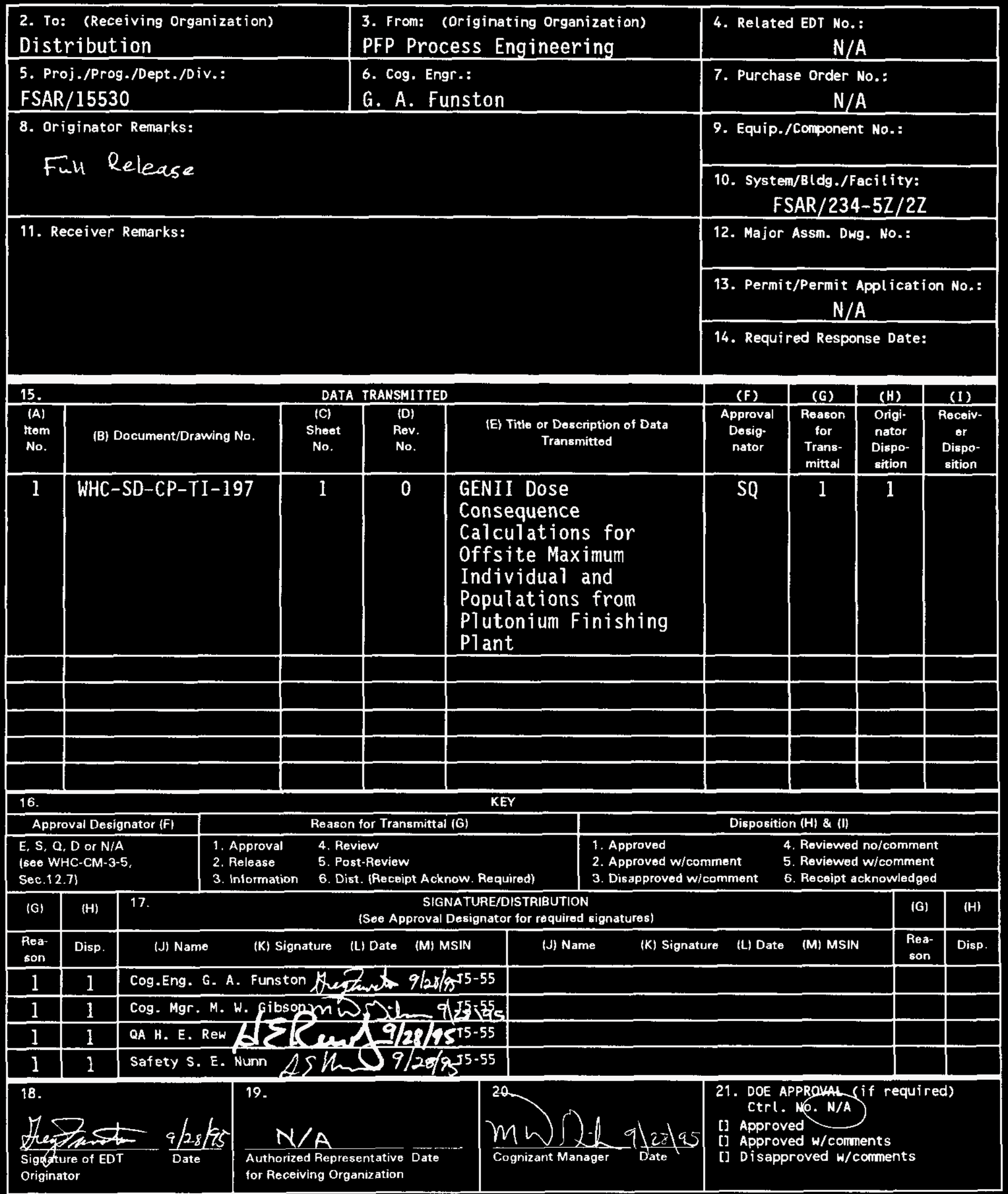


THIS PAGE INTENTIONALLY LEFT BLANK 


\section{RELEASE AUTHORIZATION}

\begin{tabular}{ll} 
Document Number: & WHC-SD-CP-TI-197, REV 0 \\
\hline Document Title: & $\begin{array}{l}\text { Geni Doser Calculations for Offsite Maximum } \\
\text { Individual and Populations from Plutonium Finishing } \\
\text { Plant }\end{array}$
\end{tabular}

Release Date: $\quad 9 / 29 / 95$

This document was reviewed following the procedures described in WHC-CM-3-4 and is:

APPROVED FOR PUBLIC RELEASE

WHC Information Release Administration Specialist:
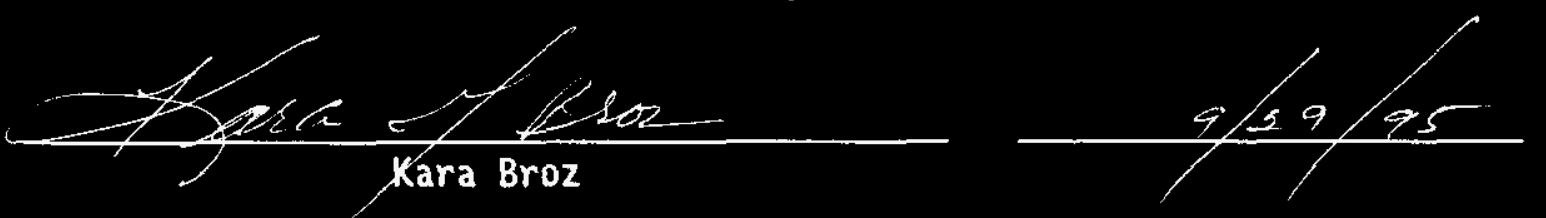

TRADEMARK DISCLAIMER. Reference here in to any specific commercial product, process, or service by trade name, trademark, manufacturer, or otherwise, does not necessarily constitute or imply its endorsement, recommendation, or favoring by the United States Government or any agency thereof or its contractors or subcontractors.

This report has been reproduced from the best available copy. Available in paper copy. Printed in the United States of America. To obtain copies of this report, contact:

Westinghouse Hanford Company - Document Control Services

P.0. Box 1970, Mailstop H6-08, Richland, WA 99352

Telephone: (509) 372-2420; Fax: (509) 376-4989 
THIS PAGE INIENTIONALIY LEFT BLANK 
2. Title

GENII DOSE CALCULATIONS FOR OFFSITE MAXIMUM

INDIVIDUAL AND POPULATIONS FROM PLUTONIUM

FINISHING PLANT

5. Key Words

FSAR, Dose Calculations, PFP
3. Number

WHC-SD-CP-TI-197
4. Rev No.

0
6. Author

Name: L. V. Nguyen Ther Punsto for L.V. Nguyen

Organization/Charge Code

\section{Abstract}

Document describes the potential dose consequences to the offsite maximum individual and population for ground and stack level releases at the offsite receptors from the Plutonium Finishing Plant.

8. 
THIS PAGE INTYANTMONALUY LEPT BLANK 
WHC-SD-CP-TI-197 Revision 0

\title{
GENII DOSE CONSEQUENCE CALCULATIONS FOR OFFSITE MAXIMUM INDIVIDUAL AND POPULATIONS FROM PLUTONIUM FINISHING PLANT
}

\author{
L. V. Nguyen \\ September 29, 1995
}

\section{PURPOSE}

Potential dose consequences to the offsite maximum individual and population for ground and stack level releases at the offsite receptors have been calculated.

\section{SOURCE TERMS}

The worst case releases for stack and ground level from the 1993 and 1991 datas, respectively, were used to calculated doses. The plutonium is assumed to be released in the form of non-oxide. The release location is to be at Plutonium Finishing Plant building.

\section{METHODOLOGIES AND ASSUMPTIONS}

The ob ectives for these problems are to calculate the maximum individual and population doses for the ground and stack level releases at offsite receptors using computer code GENII Version 1.485 (Napier et al. 1988). In addition, the dispersion coefficient values for onsite and offsite for acute and chronic (annual average) at both ground and stack level releases were included. All material released is assumed respirable. Acute and chronic release 99.5 percentile dispersion factors (X/Q) were generated using the GXQ (B. Hey, 1995) for a variety onsite occupied facilities and site boundary locations. The higher X/Q will be use for the dose calculations (NRC, Regulation Guide 1.145).

The stack downwash model was.applied (GXQ, 1993) to ground-level concentrations downwind of elevated releases. The stack height of 61 meters $(200 \mathrm{ft})$, flow rates of 260,000 $\operatorname{scfm}\left(123 \mathrm{~m}^{3} / \mathrm{s}\right)$, and inside diameters of $13.5 \mathrm{ft}$ (4.1 meters) were specified on WHC-1991. The stack height is assumed to be equivalent to a freestanding stack that is the stack height is sufficient that plumes emitted avoid entering the building wake. The stack downwash correction model (GXQ, 1993) depends upon the ratio of efflux velocity to cross-flow velocity at the top of the stack. The effluent is assumed to be at ambient temperature. 


\section{DEFINITIONS}

\section{Health Effect}

As used here, health ejiect refers to the negative health impact on a population resulting from an action which hopefully, has some benefit. It may be that the action's net result will enhance population health (i.e. reduce risk of exposure from current system configuration); however, only the negative health impact is of current concern. The only health eiject considered here is latent cancer fatality (LCF) resulting from accidental release of man-made radioactive materials.

\section{5\% Meteorology}

As used here, $99.5 \%$ meteorolo $y$ represents a condition of atmospheric dispersion which is exceeded $0.5 \%$ of the time during the year in the given sector.

\section{Offsite Population}

The $o_{i j}$ site 1 of ulation is assumed to extend from the Hanford site boundary to a circle having a 50 mile radius centered at the release point. It is assumed that the population distribution within a sector interval (i.e. area enclosed by radial and azimuthal increment) is uniform. Hanford site specific population data from the 1990 census are used.

The exposed ojisite 1 of ulation is that portion of the ojisite 1 of ulation immersed in the radioactive plume. The worst case exposed $o_{i j}$ site $l$ of ulation is the sector population which experiences the greatest combined health ejiect.

\section{OFFSITE POPULATION HEALTH EFFECT WITH 99.5\% METEOROLOGY}

Given a stack release from PFP, the $o_{j}$; site 1 of ulation health $e_{j i}$ ect is evaluated for $99.5 \%$ meteorolo $y$. The o; iste 1 of ulation is chosen to be the population in the compass sector which yields the highest population health effect. The results are reported as LCF for the worst case oij site 1 of ulation; or acute and chronic.

The worst case o; isite 1 of ulation lays in the SE sector, towards the city of Richland. This sector, out to $80,000 \mathrm{~m}$ ( $50 \mathrm{miles}$ ) has a population of 114,734 . For example, the committed effective dose equivalent (CEDE) for offsite acute ground level release to this population is calculated to be $2.9 \mathrm{E}-02$ person-rem. 
The International Commission on Radiological Protection has determined that the nominal cancer fatality coefficient for a population of all ages for low dose, low dose-rate whole body irradiation is approximately 5.0 E-04 LCF/person-rem EDE (ICRP 1991).

Assuming the accident occurs during $99.5 \%$ meteoroloky, the health ejiect to the exposed ojisite 1 of ulation chronic stack level release is given by

$$
\text { (9.0 E-04 person-rem) (5.0 E-04 LCF/person-rem) }=4.5 \text { E-07 LCF. }
$$

To place this in perspective, this exposed $o_{j}$;site 1 of ulation of 114,734 persons would normally be expected to have approximately two-hundred twenty-five LCFs per year (NSC 1990) from causes other than the accident.

The difference is approximately a factor of $5.0 \times 10^{8}$. It says that the risk to the exposed o; isite 1 of ulation chronic stack level release of a cancer fatality from all causes is 500 million times greater than the risk of a cancer fatality resulting from the postulated accident (completed populations risk of cancel fatality are included in Table 11).

\section{CODE DOCUMENTATION}

The released radionuclides are long lived and external doses (i.e. submersion and ground shine) are small in comparison to the inhalation dose. The dose due to ingestion is not included in this analysis since it is assumed that the contaminated soil would either be condemned or cleaned up following the postulated accident. This would prevent ingestion from becoming a potential exposure pathway.

The computer code GXQ Version 4.0 (Hey 1995) was used to perform the X/Q calculations necessary for the offsite and onsite population dose estimates. GXQ output files are included in Appendix B. GXQ calculates acute population-weighted atmospheric dispersion identically to GENII with one exception. GXQ sector averages the plume concentration to effectively simulate a uniform population distribution at the sector interval. Conversely, GENII calculates an acute population dose assuming centerline plume concentrations. This results in approximately one order of magnitude over-estimation by GENII.

GXQ was used to calculate $99.5 \%$ meteorology, population-weighted, sector averaged X/Qs for each sector. The sector having the largest scaled $\mathrm{X} / \mathrm{Q}$ was chosen as the worst case for population exposure in person-rem CEDE. 
WHC-SD-CP-TI-197 Revision 0

Although plume depletion was neglected, calculated worst case offsite doses could be reduced approximately $30 \%$ by considering this mechanism. All results in this analysis assumed a standard man breathing rate of $330 \mathrm{~cm}^{3} / \mathrm{s}$.

The offsite population file used by GXQ is the same as that used by GENII. Its header is given below:

"Population within $80 \mathrm{~km}$ of the Hanford 200 Area, 1990 Census (Beck, et al 1991)

Created 10/21/91 KR, Revised 2/4/92 KR"

\section{Annual Average}

The annual average $\mathrm{X} / \mathrm{Q}$ for a particular sector is calculated by summing the frequency weighted X/Qs for all atmospheric stability classes and wind speed bins in the JOINTFRE.IN (GENII, 1988) file for a particular sector. The annual average X/Q for a sector $\mathrm{K}$ is calculated using the following equation:

$$
\left(\frac{X}{Q}\right)_{k}-\sum_{j=1}^{T} \sum_{I=1}^{T}\left(\frac{X}{Q}\right)_{i j k} \frac{f_{i j k}}{C_{k}}
$$

where $\mathrm{f}_{\mathrm{jik}}$ is the annual frequency in percent that atmospheric conditions fall within stability class I, wind speed :, and sector $k$. This $X / Q$ is suitable for evaluating the consequences in sector $k$ for a chronic, year longs release. The cumulative frequency that the wind is in the direction of sector $\mathrm{k}$ is given by.

$$
C_{k}=\sum_{J=1}^{J} \sum_{I=1}^{J} f_{i j k}
$$

This value represents the annual average $\mathrm{X} / \mathrm{Q}$ of all conditions having a wind speed in the direction of sector $\mathbf{k}$. 


\section{WHC-SD-CP-TI-197 Revision 0}

\section{Chronic}

Chronic $\mathrm{X} / \mathrm{Q}$ is calculated by using : oint frequency to compute frequency to exceedance.

The frequency of exceedance $X / \mathrm{Q}^{\prime}$, refers to atmospheric conditions which are less dispersing for a specific (usually small) fraction of time. This is accomplished by sorting the sector $\mathrm{k} X / \mathrm{Qs}$ calculated for each stability class and wind speed bin such that the following equation is satisfied,

$$
\left(\frac{X}{Q}\right)_{k, 1}>\left(\frac{X}{Q}\right)_{k, 1+1}
$$

where the index $I$ includes all combinations of stability class and wind speed and $\mathrm{L}=\mathrm{I}+\mathrm{J}$. Additionally, there is a frequency, $\mathrm{f}_{\mathbf{k},}$ associated with each $(\mathrm{X} / \mathrm{Q})_{\mathrm{k} 1}$. If the percent of time the $\mathrm{X} / \mathrm{Q}^{\prime}$ is to be exceeded is specified as $\mathrm{C}_{x}$, then an index 11 is found such that

$$
C_{k, 11}<C_{x}<C_{k, 11 \cdot 1}
$$

If inorm $=F$, the cumulative frequency for sector $\mathrm{k}, \mathrm{C}_{\mathrm{k}, \mathrm{l}}$, is calculated as follows,

$$
c_{k, 11}=\sum_{1=1}^{H}\left(\frac{f_{k, 1}+f_{k, 1-1}}{2}\right)
$$

e:

.0

\footnotetext{
- joint frequency that conditions are characteri by stability class and wind speed interval 1 in sector $k(q)$
} 


\section{WHC-SD-CP-TI-197 Revision 0}

$\mathrm{C}_{\mathrm{k}, \|}$ as defined in equation 4.4-8 represents the cumulative frequency that $(\mathrm{X} / \mathrm{Q})_{\mathrm{k}, \mathrm{N}}$ is exceeded, including those times the wind is not blowing in the direction of sector $k$. The $X / Q^{\prime}$ associated with $\mathrm{C}_{\mathrm{x}}$ is found by linear interpolation such that

$$
\left(\frac{X}{Q^{\prime}}\right)_{k, 11}>\left(\frac{X}{Q^{\prime}}\right)_{k, x}>\left(\frac{X}{Q^{\prime}}\right)_{k, 11 \cdot 1}
$$

If inorm $=T$ then equation $4.4-8$ is modified as follows,

$$
C_{k, 11}-\sum_{1=1}^{H}\left(\frac{f_{k, 1}+f_{k, 1.1}}{2}\right) \frac{100}{C_{k}}
$$

The above equation for calculating the cumulative frequency is that used in the GENII code (Napier 1988); and, for consistency, the GXQ code has that capability as well. $\mathrm{C}_{\mathrm{k}, 11}$ as defined in equation 4.4-10 represents the cumulative frequency that the sector $k(X / Q)_{k, 1}$ is exceeded, excluding those times the wind is not blowing in the direction of sector $\mathrm{k} . \mathrm{C}_{\mathrm{k}}$ is defined in equation 4.4-2.

If a bounding overall site $X / Q^{\prime}$ is desired (i.e., isite $=T$ ), then the procedure described above includes all 16 sectors such that the index 1 includes all combinations of stability class, wind speed and sector and $\mathrm{L}=\mathrm{I}+\mathrm{J}+\mathrm{K}$. Calculations similar to equations 4.4-6 through 4.4-9 are solved. X/Qs are sorted such that,

$$
\left(\frac{x}{Q}\right)_{1}>\left(\frac{x}{Q}\right)_{1.1}
$$




\section{WHC-SD-CP-TI-197 Revision 0}

If the percent of time the $X / Q^{\prime}$ is to be exceeded for the overall site is specified as $C_{x}$, then an index 11 is found such that

$$
C_{11}<C_{x}<C_{11.1}
$$

where the cumulative frequency for the overall site, $\mathrm{C}_{11}$, is defined as,

$$
C_{11}=\sum_{1-1}^{1}\left(\frac{f_{1}+f_{1-1}}{2}\right)
$$

$C_{n}$ as defined in equation 4.4-13 represents the cumulative frequency that the overall site $(X / Q)_{11}$ is exceeded. The $\mathrm{X} / \mathrm{Q}^{\prime}$ associated with $\mathrm{C}_{\mathrm{x}}$ is found by linear interpolation such that

$$
\left(\frac{x}{Q^{\prime}}\right)_{11}>\left(\frac{x}{Q^{\prime}}\right)_{x}>\left(\frac{x}{Q^{\prime}}\right)_{11,1}
$$

Again, setting inorm $=T$ in addition to setting isite $=T$ has no effect.

\section{Population Weighting}

Population weighting $X / Q$ for each sector, stability class, and wind speed bin, $(X / Q)_{i j k}$ is calculated as follows:

$$
\left(\frac{x}{Q}\right)_{i j k}-\sum_{m=1}^{M}\left(\frac{x}{Q}\right)_{i j k} P_{k m}
$$

where:

$$
\mathbf{M}=\text { number of radial intervals }
$$




\section{WHC-SD-CP-TI-197 Revision 0}

$$
\mathbf{P}_{\mathrm{km}}=\text { population contained in sector } \mathrm{k} \text {, radial interval } \mathrm{m} \text {. }
$$

The radial intervals are defined by their midpoint distances specified in the population file POP.IN.

\section{ATMOSPHIRIC DISPERSION COEFFICIENT - X/Q}

After the radioactive material becomes airborne, it is transported downwind and could potentially be inhaled by the public. The concentration of this material is reduced, or diluted, as it is being transported due to atmospheric mixing and turbulence. An atmospheric relative concentration value $(\mathrm{X} / \mathrm{Q})$ is used to characterized the dilution of the airborne contaminants during atmospheric transport and dispersion of the airborne contaminants during atmospheric transport and dispersion. $\mathrm{X} / \mathrm{Q}$ is a function of the atmospheric conditions (i.e., wind speed, stability class) and the distance to the receptor.

The Gaussian straight line model is employed by GXQ for calculating atmospheric dispersion. The time integrated atmospheric dispersion coefficient $\mathrm{X} / \mathrm{Q}$ for a continuous point source strength is given by:

$$
\frac{X}{Q} \cdot \frac{f(y) g(z)}{2 \pi \sigma_{y} \sigma_{z} \bar{U}}
$$

where:

$$
\begin{array}{lll}
\sigma_{\mathrm{y}} & = & \text { Horizontal diffusion coefficient }(\mathrm{m}) \\
\boldsymbol{\sigma}_{\mathrm{x}} & = & \text { Vertical diffusion coefficient }(\mathrm{m}) \\
\mathrm{f}(\mathrm{y}) & = & \text { Horizontal corrections term } \\
\mathrm{g}(\mathrm{z}) & = & \text { Vertical corrections term } \\
\overline{\mathrm{U}} & = & \text { Mean wind speed }(\mathrm{m} / \mathrm{s})
\end{array}
$$

The horizontal correction term is given by:

$$
f(y)-\exp \left\{\frac{1}{2}\left\{\frac{y}{\sigma_{y}}\right\}\right.
$$

where:

$$
\mathrm{y}=\text { Horizontal offset from a plume centerline (m) }
$$




\section{WHC-SD-CP-TI-197 Revision 0}

If the mixing height is large compared to the release height and the vertical diffusion coefficient, then the vertical correction term is given by:

$$
g(z) \cdot F_{r e f} \exp \left\{-\frac{1}{2}\left\{\frac{h_{e}-z}{\sigma_{z}}\right\}^{2}\right\}
$$

where:

$$
\begin{array}{lll}
\text { Fref } & = & \text { Reflection factors } \\
\mathbf{h}_{\mathbf{c}} & = & \text { Effective releases height }(\mathrm{m}) \\
\mathbf{z} & = & \text { Receptor height }(\mathrm{m}) .
\end{array}
$$

Mathematical approximations are used to evaluate the atmospheric diffusion coefficients $\sigma_{y} \& \sigma_{z}$ which are based on the Pasquil-Gifford-Turner curves. These approximations are identical to those used in the GENII Code (Napier, 1988).

\section{APPLICATIONS} materials.

This section describes how $\mathrm{X} / \mathrm{Q}$ is used to estimate the dose from a release of radioactive

The $X / Q$ refers to the concentration of airborne contamination. It is the ratio of contaminant concentration at a specific receptor location to a contaminant release rate. The receptor of concern is normally defined as a human having a standard breathing rate and metabolism. The standard man breathing rates are typically taken from ICRP 23 (1975) as shown below:

\section{Release scenario}

Acute release (i.e., less than 24 hours)

Chronic release (i.e., greater than 24 hours)

\section{Breathing rates}

$$
3.3 \mathrm{E}-4 \mathrm{~m} 3 / \mathrm{s}
$$

\section{$2.7 \mathrm{E}-4 \mathrm{~m} 3 / \mathrm{s}$}

The product of X/Q and breathing rate is a unitless number representing the fraction of contaminant inhaled to contaminant released. Inhalation is by far the most significant, unavoidable, and likely pathway to human exposure. Although ingestion represents an additional theoretical pathway, the ingestion pathway can be avoided through interdiction of 
WHC-SD-CP-TI-197 Revision 0

food products if necessary in the event of an accident. The ground shine and plume submersion pathways can, for all practical purposes, be ignored for releases of plutonium.

The dose from the inhalation of radioactive materials, plutonium in particular, can be calculated using the following formula:

$$
D\left(\text { rem) }-Q(g) \times \frac{X}{Q}\left(s / m_{3}\right) \times R\left(m^{3} / s\right) \times C(\text { rem } / g)\right.
$$

Where:
$Q=$ Quantity plutonium released
$\chi / Q=$ Atmospheric dispersion coefficient
$\mathbf{R}=$ Breathing rate
C $=$ Gram plutonium to dose conversion factor.

The quantity released, $Q$, is specific to the accident scenario. It is a function of several factors, including the inventory at risk, dispersing energy available, release fraction, respirable fraction, and mitigation. It is essentially a source term represented in grams. The grams plutonium to dose conversion factor, $\mathrm{C}$, is specific to the istopic mixture and chemical compound under consideration. The Technical Basis for Characterization of Plutonium for PFP Safety Analyses (Crowe and Szempruch 1994) contains such information for the inhalation pathway. Conversion factors for both oxides and nonoxides are provided for both the committed effective dose equivalent and the maximum organ committed dose.

\section{RECEPTOR DESCRIPTIONS}

- Maximum Individual Onsite: Doses calculated for the onsite receptor include inhalation and submersion. Ground level release dispersion factors $(\mathrm{X} / \mathrm{Q})$ were generated using the GXQ code for surrounding occupied facility around the PFP. These results are summarized in the Tables 1 and 2 . The release location will be determined based on the largest $\mathrm{X} / \mathrm{Q}$ value. The single asterisks in the tables identify the worst $\mathrm{X} / \mathrm{Q}$ which will be used for dose calculations.

- Maximum Individual Site Boundary: Receptors at the site boundary in the worst direction. Where the site is bounded by the Columbia River, the site boundary is taken to be at the nearer bank of the river. This receptor is assumed to stay at this location for the duration of the accident. Doses calculated include inhalation and submersion. Atmosphere dispersion factors $(\mathrm{X} / \mathrm{Q})$ were generated for 16 sectors location around the 


\section{WHC-SD-CP-TI-197 Revision 0}

site boundary. These results are summarized in the Table 3 . The single asterisks in the table identify the worst $X / Q$ which will be used for dose calculations.

\section{CODE DOCUMENTATION}

- GENII version $1.485(12 / 3 / 90)$

- GENII Default Parameter Values (28-Mar-90 RAP)

- Radionuclide Master Library (7/23/93 PDR)

- PNL Food Transfer Factor Library (7/19/93)

- External Dose Factor Library (8-May-90-RAP)

- Internal Dose Increments, Maximum Worst Case Solubilities, (7/23/93 PDR)

- Joint Frequency Data:

200 Area, 10 m, Pasquill A-G (1983-1991 Average)

200 Area, $61 \mathrm{~m}$, Pasquill A-G (1983-1991 Average)

200 Area, 89 m, Pasquill A-G (1983-1991 Average)

- GXQ Version 4 (1995)

Typical GENII and GXQ input files are attached in Appendix A and B.

NOTE 1: Please, be aware of the jact that the new , oint irequency data, and NRC Refulatory Guide 1.145 atmosy heric disy ersion coej) icients $(\lambda, Q$ methods were $a_{1}$ lied jor dose calculations. These chang es may ej ect consistency with I revious $\lambda, Q$ s and dose consequences. This document was perjormed based on the best available injormation at this time.

\section{RESULTS}

The resulting EDE and limiting organ doses for onsite and site boundary receptors calculated by GENII 1.485 for stack and ground level releases are shown in the Tables 7, 8, and 9. Inhalation doses were 50-year committed doses based on an acute uptake. Submersion doses were calculated using a semi-infinite cloud model. 


\section{WHC-SD-CP-TI-197 Revision 0}

In addition, population health effects in terms of latent cancer fatalities (LCF) were determined and are reported. The health effect results are summarized in Table 11. 
WHC-SD-CP-TI-197 Revision 0

Table 1: Maximum Individual with an Acute and Chronic at Onsite Nearest Occupied Facilities for Ground Level Release ( 99.5 Percentile X/Q).

\begin{tabular}{|c|c|c|c|c|}
\hline Building & Direction & Distance $(\mathrm{m})$ & Acute $\left(\mathrm{s} / \mathrm{m}^{3}\right)$ & Chronic $\left(\mathrm{s} / \mathrm{m}^{3}\right)$ \\
\hline 272-WA & WNW & 550 & $8.72 \mathrm{E}-04^{*}$ & $6.00 \mathrm{E}-06 *$ \\
\hline T-PLANT & NE & 1540 & $1.59 \mathrm{E}-04$ & $7.92 \mathrm{E}-07$ \\
\hline 2713-WB & ENE & 750 & $8.22 \mathrm{E}-04$ & $3.65 \mathrm{E}-06$ \\
\hline U-PLANT & ESE & 1050 & $6.03 \mathrm{E}-04$ & $5.81 \mathrm{E}-06$ \\
\hline 242-S & SSE & 930 & $3.59 \mathrm{E}-04$ & $2.47 \mathrm{E}-06$ \\
\hline
\end{tabular}

Table 2: Maximum Individual with an Acute and Chronic at Onsite Nearest Occupied Facilities for Stack Level Release (99.5 Percentile X/Q).

\begin{tabular}{|c|c|c|c|c|}
\hline Building & Direction & Distance $(\mathrm{m})$ & Acute $\left(\mathrm{s} / \mathrm{m}^{3}\right)$ & Chronic $\left(\mathrm{s} / \mathrm{m}^{3}\right)$ \\
\hline 272-WA & WNW & 630 & $5.57 \mathrm{E}-06^{*}$ & $5.63 \mathrm{E}-08 *$ \\
\hline T-PLANT & NE & 1580 & $1.03 \mathrm{E}-06$ & $8.90 \mathrm{E}-09$ \\
\hline $2713-W B$ & ENE & 830 & $2.16 \mathrm{E}-06$ & $2.42 \mathrm{E}-08$ \\
\hline U-PLANT & ESE & 1060 & $2.52 \mathrm{E}-06$ & $2.77 \mathrm{E}-08$ \\
\hline $242-S$ & SSE & 930 & $3.35 \mathrm{E}-06$ & $3.28 \mathrm{E}-08$ \\
\hline
\end{tabular}




\section{WHC-SD-CP-TI-197 Revision 0}

Table 3: 99.5 Percentile X/Qs Values and 95 Percentile X/Qs at Site Boundary Locations for a Maximum Individual Ground and Stack Level for Acute and Chronic Releases.

\begin{tabular}{|c|c|c|c|c|c|}
\hline \multirow[t]{2}{*}{ Sector } & \multirow{2}{*}{$\begin{array}{c}\text { Distance } \\
\text { (km) }\end{array}$} & \multicolumn{2}{|c|}{ Ground Level Release } & \multicolumn{2}{|c|}{ Stack Level Release } \\
\hline & & $\mathrm{X} / \mathrm{Q}\left(\mathrm{s} / \mathrm{m}^{3}\right)$ Acute & $X / Q\left(s / \mathrm{m}^{3}\right)$ Chronic & $\mathrm{X} / \mathrm{Q}\left(\mathrm{s} / \mathrm{m}^{3}\right)$ Acute & $\mathrm{X} / \mathrm{Q}\left(\mathrm{s} / \mathrm{m}^{3}\right)$ Chronic \\
\hline $\mathbf{S}$ & 15.0 & $6.71 \mathrm{E}-06$ & $3.62 \mathrm{E}-08$ & $5.28 \mathrm{E}-07$ & $9.69 \mathrm{E}-09$ \\
\hline SSW & 15.4 & 4.10 E-06 & $2.09 \mathrm{E}-08$ & $4.32 \mathrm{E}-07$ & $6.05 \mathrm{E}-09$ \\
\hline SW & 16.1 & $4.97 \mathrm{E}-06$ & $1.94 \mathrm{E}-08$ & $4.10 \mathrm{E}-07$ & $4.99 \mathrm{E}-09$ \\
\hline WSW & 13.2 & $6.40 E-06$ & $2.57 \mathrm{E}-08$ & $5.10 \mathrm{E}-07$ & $5.86 \mathrm{E}-09$ \\
\hline $\mathrm{W}$ & 12.5 & $1.62 \mathrm{E}-05^{*}$ & $5.02 \mathrm{E}-08^{*}$ & $6.24 \mathrm{E}-07 *$ & $1.03 \mathrm{E}-08 *$ \\
\hline WNW & 13.2 & $8.49 \mathrm{E}-06$ & $3.85 \mathrm{E}-08$ & $5.85 \mathrm{E}-07$ & $7.83 \mathrm{E}-09$ \\
\hline NW & 16.5 & $1.18 \mathrm{E}-05$ & $3.29 \mathrm{E}-08$ & $5.10 \mathrm{E}-07$ & $7.04 \mathrm{E}-09$ \\
\hline NNW & 17.4 & $1.11 \mathrm{E}=05$ & $3.12 \mathrm{E}-08$ & $5.12 \mathrm{E}-07$ & $6.23 \mathrm{E}-09$ \\
\hline $\mathbf{N}$ & 19.2 & $1.05 \mathrm{E}-05$ & $2.91 \mathrm{E}-08$ & $4.82 \mathrm{E}-07$ & 5.47 E-09 \\
\hline NNE & 26.0 & $3.15 \mathrm{E}-06$ & $1.07 \mathrm{E}-08$ & $2.01 \mathrm{E}-07$ & $2.37 \mathrm{E}-09$ \\
\hline $\mathrm{NE}$ & 28.9 & $2.98 \mathrm{E}-06$ & $1.09 \mathrm{E}-08$ & $1.95 \mathrm{E}-07$ & $2.65 \mathrm{E}-09$ \\
\hline ENE & 25.6 & $6.02 \mathrm{E}-06$ & $1.72 \mathrm{E}-08$ & $2.36 \mathrm{E}-07$ & $3.92 \mathrm{E}-09$ \\
\hline $\mathrm{E}$ & 25.2 & $8.21 \mathrm{E}-06$ & $4.82 \mathrm{E}-08$ & $4.35 \mathrm{E}-07$ & $9.21 \mathrm{E}-09$ \\
\hline ESE & 30.0 & $6.34 \mathrm{E}-06$ & $3.95 \mathrm{E}-08$ & 3.42 E- 07 & 9.47 E-09 \\
\hline SE & 25.2 & $7.44 \mathrm{E}-06$ & $2.95 \mathrm{E}-08$ & $3.60 \mathrm{E}-07$ & $7.86 \mathrm{E}-09$ \\
\hline SSE & 22.9 & $4.03 \mathrm{E}-06$ & $1.85 \mathrm{E}-08$ & $3.40 \mathrm{E}-07$ & $5.29 \mathrm{E}-09$ \\
\hline
\end{tabular}


Table 4: 99.5 Percentile X/Qs Values at Site Boundary Locations for a Population Ground and Stack Level for Acute and Chronic Releases at $80 \mathrm{~km}$ Around 16 Sectors.

\begin{tabular}{|c|c|c|c|c|}
\hline \multirow[t]{2}{*}{ Sector } & \multicolumn{2}{|c|}{ Ground Level Release } & \multicolumn{2}{|c|}{ Stack Level Release } \\
\hline & $\mathrm{X} / \mathrm{Q}\left(\mathrm{s} / \mathrm{m}^{3}\right)$ Acute & $\mathrm{X} Q\left(\mathrm{~s} / \mathrm{m}^{3}\right)$ Chronic & $\mathrm{X} / \mathrm{Q}\left(\mathrm{s} / \mathrm{m}^{3}\right)$ Acute & $\mathrm{X} / \mathrm{Q}\left(\mathrm{s} / \mathrm{m}^{3}\right)$ Chronic \\
\hline $\mathbf{S}$ & $3.37 \mathrm{E}-03$ & $7.40 \mathrm{E}-05$ & $1.54 \mathrm{E}-03$ & $2.61 \mathrm{E}-05$ \\
\hline SSW & $3.59 \mathrm{E}-03$ & $7.77 \mathrm{E}-05$ & $2.05 \mathrm{E}-03$ & $3.20 \mathrm{E}-05$ \\
\hline SW & $6.59 \mathrm{E}-03$ & $1.27 \mathrm{E}-04$ & $3.42 \mathrm{E}-03$ & $4.61 \mathrm{E}-05$ \\
\hline WSW & $6.66 \mathrm{E}-03$ & $1.27 \mathrm{E}-04$ & $3.47 \mathrm{E}-03$ & $4.87 \mathrm{E}-05$ \\
\hline W & $2.21 \mathrm{E}-02$ & $5.01 \mathrm{E}-04$ & $1.37 \mathrm{E}-02$ & $2.02 \mathrm{E}-04$ \\
\hline WNW & $1.80 \mathrm{E}-03$ & $3.52 \mathrm{E}-05$ & $8.06 \mathrm{E}-04$ & $1.22 \mathrm{E}-05$ \\
\hline NW & $1.21 \mathrm{E}-03$ & $2.64 \mathrm{E}-05$ & $5.73 \mathrm{E}-04$ & $8.24 \mathrm{E}-06$ \\
\hline NNW & $2.22 \mathrm{E}-03$ & $4.86 \mathrm{E}-05$ & $1.28 \mathrm{E}-03$ & $1.59 \mathrm{E}-05$ \\
\hline $\mathrm{N}$ & $2.00 \mathrm{E}-03$ & $3.68 \mathrm{E}-05$ & $9.05 \mathrm{E}-04$ & $1.07 \mathrm{E}-05$ \\
\hline NNE & $5.28 \mathrm{E}-03$ & $7.73 \mathrm{E}-05$ & $1.88 \mathrm{E}-03$ & $2.43 \mathrm{E}-0.5$ \\
\hline $\mathrm{NE}$ & $3.85 \mathrm{E}-03$ & $6.40 \mathrm{E}-05$ & $1.35 \mathrm{E}-03$ & $1.81 \mathrm{E}-05$ \\
\hline ENE & $1.48 \mathrm{E}-03$ & $3.20 \mathrm{E}-05$ & $5.58 \mathrm{E}-04$ & $8.95 \mathrm{E}-06$ \\
\hline $\mathrm{E}$ & $2.66 \mathrm{E}-03$ & $9.06 \mathrm{E}-05$ & $9.79 \mathrm{E}-04$ & $2.18 \mathrm{E}-05$ \\
\hline ESE & $1.83 \mathrm{E}-03$ & $7.38 \mathrm{E}-05$ & $6.60 \mathrm{E}-04$ & $1.98 \mathrm{E}-05$ \\
\hline SE & $4.86 \mathrm{E}-02$ * & $1.36 \mathrm{E}-03$ * & $1.85 \mathrm{E}-02 *$ & $4.55 \mathrm{E}-04 *$ \\
\hline SSE & $7.66 \mathrm{E}-03$ & $1.55 \mathrm{E}-04$ & $2.85 \mathrm{E}-03$ & $5.34 \mathrm{E}-05$ \\
\hline
\end{tabular}


WHC-SD-CP-TI-197 Revision 0

Table 5: Summazied The Maximum Individual Worst Cases 99.5 Percentile X/Qs for Onsite and Offsite.

\begin{tabular}{|c|c|c|}
\hline Location of release & Acute Release $\left(\mathrm{s} / \mathrm{m}^{3}\right)$ & Chronic Release $\left(\mathrm{s} / \mathrm{m}^{3}\right)$ \\
\hline Onsite Ground level & $8.72 \mathrm{E}-04(550 \mathrm{~m}, \mathrm{WNW})$ & $6.00 \mathrm{E}-06(550 \mathrm{~m}, \mathrm{WNW})$ \\
\hline Onsite Stack level & $5.57 \mathrm{E}-06(630 \mathrm{~m}, \mathrm{WNW})$ & $5.63 \mathrm{E}-08(630 \mathrm{~m}, \mathrm{WNW})$ \\
\hline Offsite Ground level & $1.62 \mathrm{E}-05(12.5 \mathrm{~km} \mathrm{~W})$ & $5.02 \mathrm{E}-08(12.5 \mathrm{~km}, \mathrm{~W})$ \\
\hline Offsite Stack level & $6.24 \mathrm{E}-07(12.5 \mathrm{~km} \mathrm{~W})$ & $1.03 \mathrm{E}-08(12.5 \mathrm{~km}, \mathrm{~W})$ \\
\hline
\end{tabular}

Table 6: Summazied The Population Weighted Worst Cases

99.5 Percentile X/Qs for Onsite and Offsite at $80 \mathrm{~km}$.

\begin{tabular}{|c|c|c|}
\hline Location of release & Acute Release $\left(\mathrm{s} / \mathrm{m}^{3}\right)$ & Chronic Release $\left(\mathrm{s} / \mathrm{m}^{3}\right)$ \\
\hline Offsite Ground level & $4.86 \mathrm{E}-02(\mathrm{SE})$ & $1.36 \mathrm{E}-03(\mathrm{SE})$ \\
\hline Offsite Stack level & $1.85 \mathrm{E}-02(\mathrm{SE})$ & $4.55 \mathrm{E}-04(\mathrm{SE})$ \\
\hline
\end{tabular}




\section{WHC-SD-CP-TI-197 Revision 0}

Table 7. Maximum Individual Doses Calculated for an

Acute and Chronic Offsite Stack Level Release, Inventory from 1993, 291-Z-1 Elevated Release.

\begin{tabular}{|c|c|c|cc|}
\hline \multirow{2}{*}{$\begin{array}{c}\text { Release Type } \\
\text { Chronic } \\
\mathrm{X} / \mathrm{Q}=1.03 \mathrm{E}-08\end{array}$} & Dose type & EDE (rem) & \multicolumn{2}{c|}{ Limiting organ (rem) } \\
\cline { 2 - 5 } & Inhalation & $1.0 \mathrm{E}-06$ & $1.9 \mathrm{E}-05$ & (Bone Sur) \\
\hline & Submersion & $2.2 \mathrm{E}-15$ & $2.2 \mathrm{E}-15$ & \\
\hline \multirow{2}{*}{$\begin{array}{c}\text { Acute } \\
\mathrm{X} / \mathrm{Q}=6.24 \mathrm{E}-07\end{array}$} & Inhalation & $1.0 \mathrm{E}-06$ & $1.9 \mathrm{E}-05$ & (Bone Sur) \\
\cline { 2 - 5 } & Submersion & $6.1 \mathrm{E}-05$ & $1.1 \mathrm{E}-03$ & (Bone Sur) \\
\hline & $1.3 \mathrm{E}-13$ & $1.3 \mathrm{E}-13$ & \\
\hline
\end{tabular}

EDE effective dose equivalent.

Bone Sur bone surface.

Table 8. Population Doses Calculated for an Acute and Chronic Offsite Stack Level Release, Unit Are in person-rem, Inventory from 1993, 291-Z-1 Elevated Release.

\begin{tabular}{|c|c|c|c|c|}
\hline Release Type & Dose type & EDE & \multicolumn{2}{|c|}{ Limiting organ } \\
\hline \multirow{2}{*}{$\begin{array}{c}\text { Chronic } \\
\mathrm{X} / \mathrm{Q}=4.55 \mathrm{E}-04\end{array}$} & Inhalation & 4.4 E-02 & 8.2 E-01 & (Bone Sur) \\
\hline & Submersion & $9.6 \mathrm{E}-11$ & $9.6 \mathrm{E}-11$ & \\
\hline & Total & $4.4 \mathrm{E}-02$ & 8.2 E-01 & (Bone Sur) \\
\hline \multirow{2}{*}{$\begin{array}{c}\text { Acute } \\
\mathrm{X} / \mathrm{Q}=1.85 \mathrm{E}-02\end{array}$} & Inhalation & $1.8 \mathrm{E}+00$ & $3.3 \mathrm{E}+01$ & (Bone Sur) \\
\hline & Submersion & $3.9 \mathrm{E}-09$ & $3.9 \mathrm{E}-09$ & \\
\hline & Total & $1.8 \mathrm{E}+00$ & $3.3 \mathrm{E}+01$ & (Bone Sur) \\
\hline
\end{tabular}

EDE effective dose equivalent.

Bone Sur bone surface. 
WHC-SD-CP-TI-197 Revision 0

Table 9. Maximum Individual Doses Calculated for an

Acute and Chronic Offsite Ground Level Release, Inventory from 1991, 296-Z-3 Ground Release.

\begin{tabular}{|c|c|c|c|c|}
\hline Release Type & Dose type & EDE (rem) & \multicolumn{2}{|c|}{ Limiting organ (rem) } \\
\hline \multirow{2}{*}{$\begin{array}{c}\text { Chronic } \\
X / Q=5.02 \mathrm{E}-08\end{array}$} & Inhalation & $3.0 \mathrm{E}-08$ & $5.5 \mathrm{E}-07$ & (Bone Sur) \\
\hline & Submersion & $5.0 \mathrm{E}-17$ & $5.0 \mathrm{E}-17$ & \\
\hline \multicolumn{2}{|r|}{ Total } & $3.0 \mathrm{E}-08$ & $5.5 \mathrm{E}-07$ & (Bone Sur) \\
\hline \multirow{2}{*}{$\begin{array}{c}\text { Acute } \\
\mathrm{X} / \mathrm{Q}=1.62 \mathrm{E}-05\end{array}$} & Inhalation & $9.6 \mathrm{E}-06$ & $1.8 \mathrm{E}-04$ & (Bone Sur) \\
\hline & Submersion & $1.6 \mathrm{E}-14$ & $1.6 \mathrm{E}-14$ & \\
\hline \multicolumn{2}{|r|}{ Total } & 9.6 E-06 & $1.8 \mathrm{E}-04$ & (Bone Sur) \\
\hline
\end{tabular}

EDE effective dose equivalent.

Bone Sur bone surface.

Table 10. Population Doses Calculated for an Acute and Chronic Offsite Ground Level Release, Unit Are in person-rem, Inventory from 1991, 296-Z-3 Ground Release.

\begin{tabular}{|c|c|c|cc|}
\hline Release Type & Dose type & EDE & \multicolumn{2}{|c|}{ Limiting organ } \\
\hline \multirow{2}{*}{$\begin{array}{c}\text { Chronic } \\
\mathrm{X} / \mathrm{Q}=1.36 \mathrm{E}-03\end{array}$} & Inhalation & $8.0 \mathrm{E}-04$ & $1.5 \mathrm{E}-02$ & (Bone Sur) \\
\cline { 2 - 6 } & Submersion & $1.4 \mathrm{E}-12$ & $1.4 \mathrm{E}-12$ & \\
\hline \multicolumn{2}{c}{ Total } & $8.0 \mathrm{E}-04$ & $1.5 \mathrm{E}-02$ & (Bone Sur) \\
\hline $\mathrm{X} / \mathrm{Q}=4.86 \mathrm{E}-02$ & Inhalation & $2.9 \mathrm{E}-02$ & $5.4 \mathrm{E}-01$ & (Bone Sur) \\
\cline { 2 - 6 } & Submersion & $4.9 \mathrm{E}-11$ & $4.9 \mathrm{E}-11$ & \\
\hline \multicolumn{2}{r}{ Total } & $2.9 \mathrm{E}-02$ & $5.4 \mathrm{E}-01$ & (Bone Sur) \\
\hline
\end{tabular}

EDE effective dose equivalent.

Bone Sur bone surface. 
WHC-SD-CP-TI-197 Revision 0

Table 11: Worst Case Ofisite Population (114,734) Bealth Effects in SE.

\begin{tabular}{|c|c|c||}
\hline Receptor & Health Effect & Risk to the exposed $^{2}$ \\
\hline Acute Ground Level Release & $1.5 \mathrm{E}-05 \mathrm{LCF}$ & $3.00 \mathrm{E}+09$ \\
\hline Chronic Ground Level Release & $4.0 \mathrm{E}-07 \mathrm{LCF}$ & $1.13 \mathrm{E}+10$ \\
\hline Acute Stack Level Release & $2.2 \mathrm{E}-05 \mathrm{LCF}$ & $\cdot 2.05 \mathrm{E}+08$ \\
\hline Chronic Stack Level Release & $9.0 \mathrm{E}-04 \mathrm{LCF}$ & $5.00 \mathrm{E}+08$ \\
\hline
\end{tabular}

a Risk of a cancer fatality. 


\section{WHC-SD-CP-TI-197 Revision 0}

\section{REFERENCES}

Napier, B. A., et al., 1988, GEM, - The Han;ord Environmental Radiation Dosimetry So; tware System, PNL-6484, Pacific Northwest Laboratory, Richland, Washington.

NSC, 1990, National Safety Council, Accident Facts - 1990 Edition, Chicago Illinois, p. 6.

ICRP, 1991, „nternational Commission on Radiological Protection, Annals o; the ,CRP, Publication 60, 1991, p. 132-133.

Hey, B. E., 1995, GXQ Proł ram Users' Guide, WHC-SD-GN-SWD-30002, Westinghouse Hanford Company, Richland, Washington.

U.S. Nuclear Regulatory Commission, November 1982, Atmosy heric Disy ersion N odels For Potential Accident Consequence Assessments At $\Lambda$ uclear Power Plants, Regulatory Guide 1.145, Revision 1, Office Of Nuclear Regulatory Research.

WHC-CM-4-46, Nonreactor Facility Sajety Analysis $N$ anual, Westinghouse Hanford Company, Richland, Washington. 


\section{WHC-SD-CP-TI-197 Revision 0}

\section{APPENDIX A}

Sample GENII Input \& Output Decks for X/Q $=1.0 \mathrm{~s} / \mathrm{m}^{3}$ 



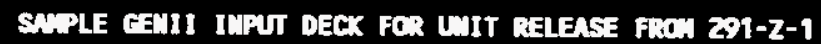
STACX LEVL RDIOAGTIVE AIRGODUE RELEASE FROA $19 \% 3$ DATA.

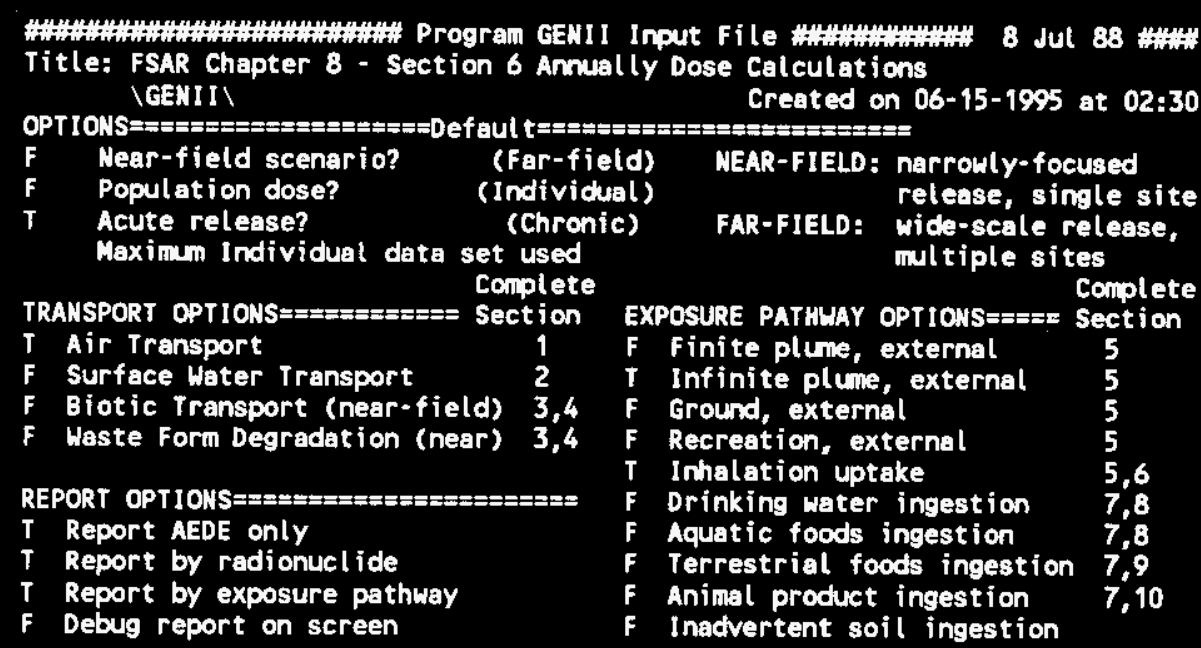

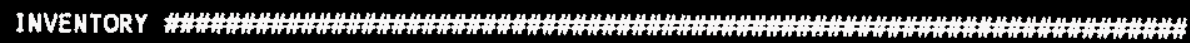

4 Inventory input activity units: (1-pCi $2-u C j \quad 3-m c i \quad 4-C i \quad 5-8 q)$

o Surface soil source units (1- $\mathrm{m}^{2} 2-\mathrm{mb}^{3} 3-\mathrm{kg}$ )

Equil ibriun question goes here

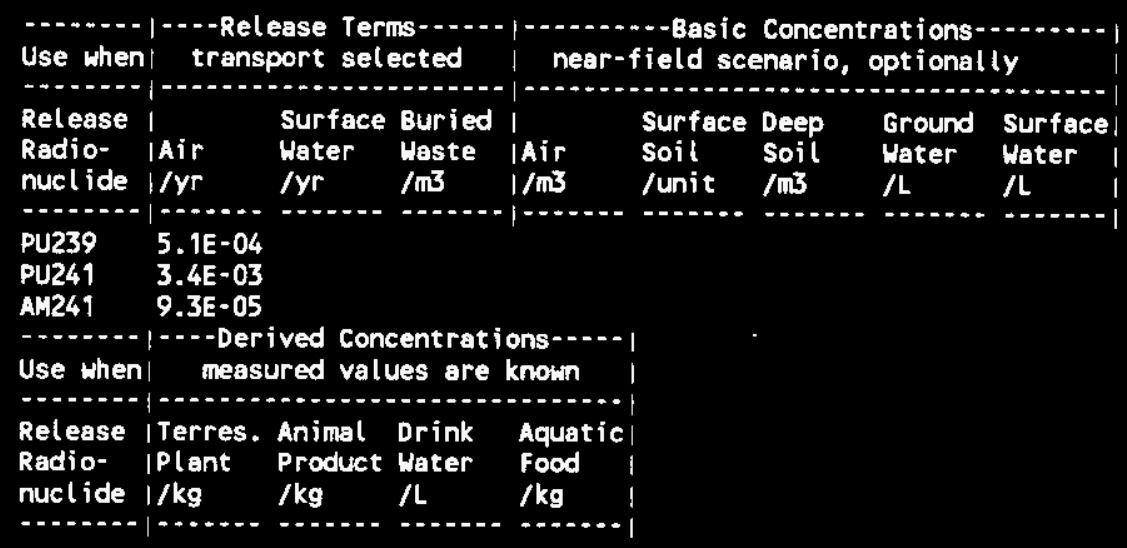

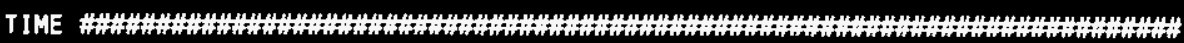

$1 \quad$ Intake ends after (yr)

50 Dose calc. ends after (yr)

Release ends after ( $y r)$

No. of years of air deposition prior to the intake period

No. of years of irrigation water deposition prior to the intake period

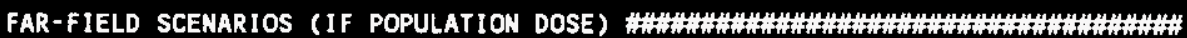

0 Definition option: 1-Use population grid in file PoP.IN

$0 \quad 2$-Use total entered on this line 


\section{WHC-SD-CP-TI-197 Revision 0}

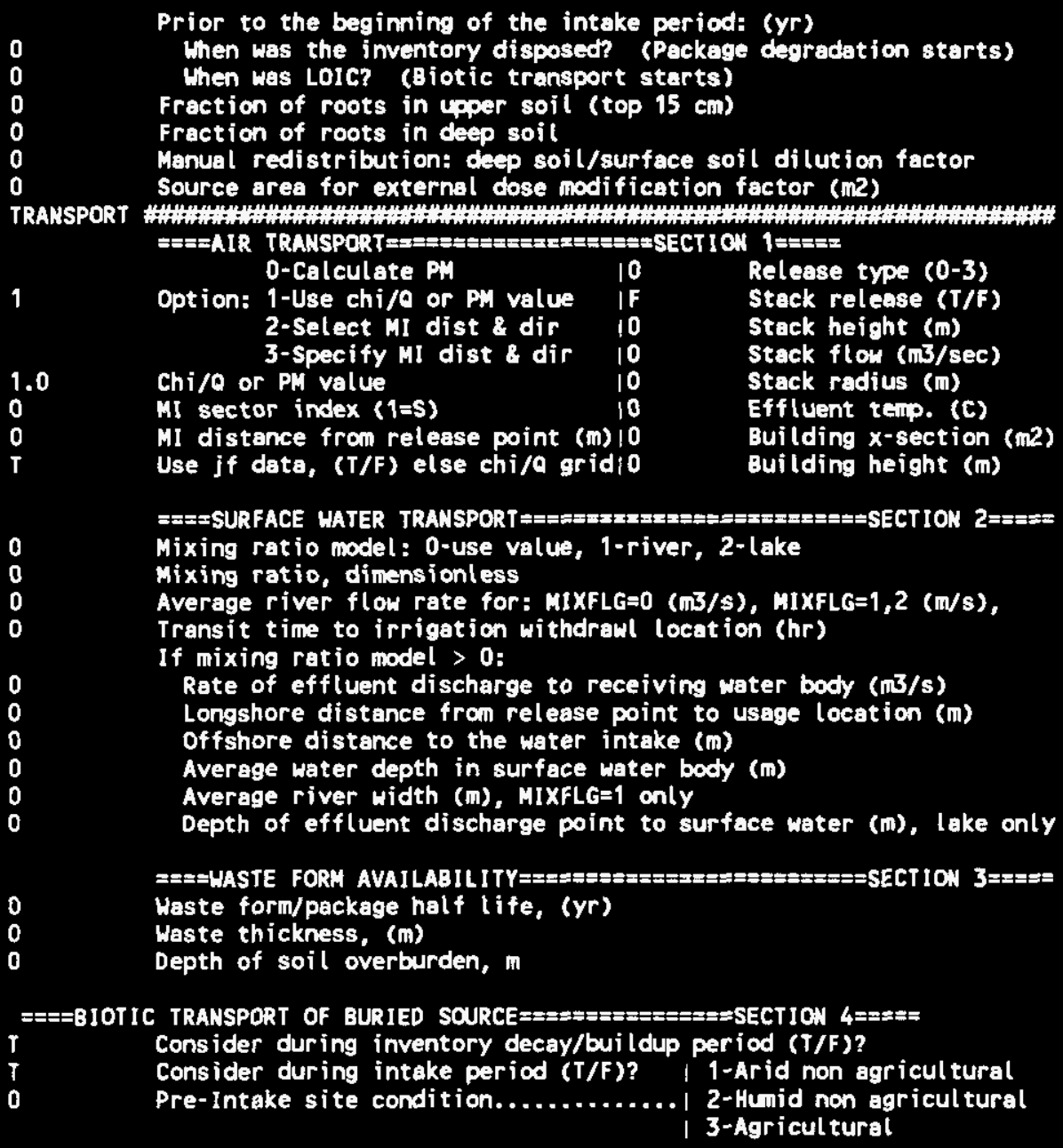

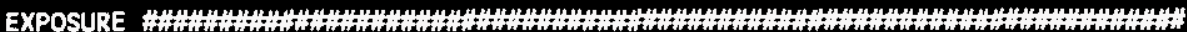

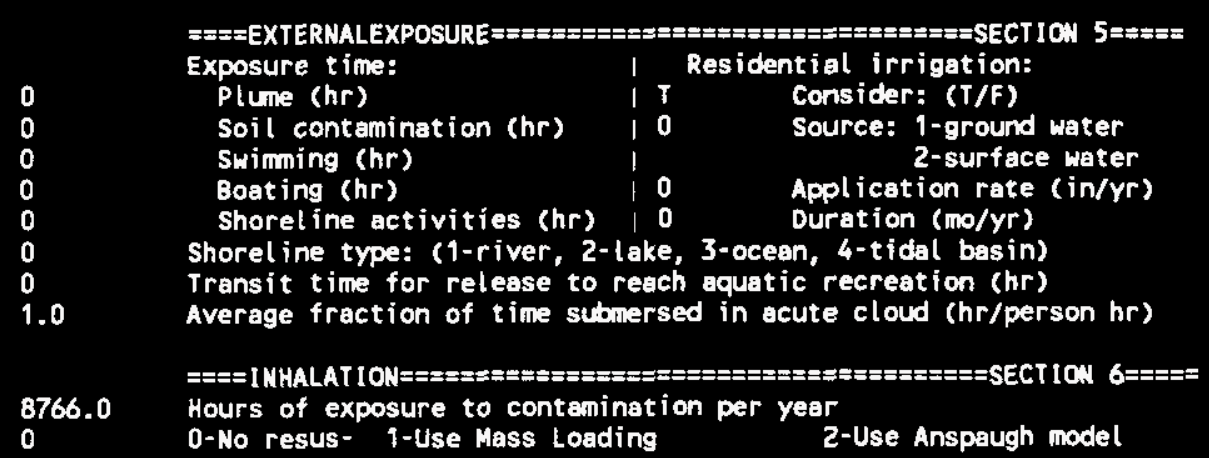




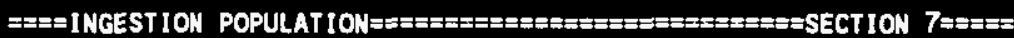
Atmospheric production definition (select option):

0 -Use food-ueighted chi/a, (food-sec/m3), enter value on this line

1-Use population-weighted chi/o

2-Use uniform production

3-Use chi/a and production grids (PRODUCTION will be overridden) Population ingesting aquatic foods, 0 defaults to total (person) population ingesting drinking water, 0 defaults to total (person) Consider dose from food exported out of region (defaul $t=F$ )

Note below: $\mathrm{s}^{*}$ or Source: 0 -none, 1-ground water, 2-surface water 3-Derived concentration entered above $== \pm=$ AQUATIC FOOOS / DRINKING WATER INGESTION $== \pm== \pm= \pm=5$ SECTION $8== \pm=$ Salt water? (default is fresh)

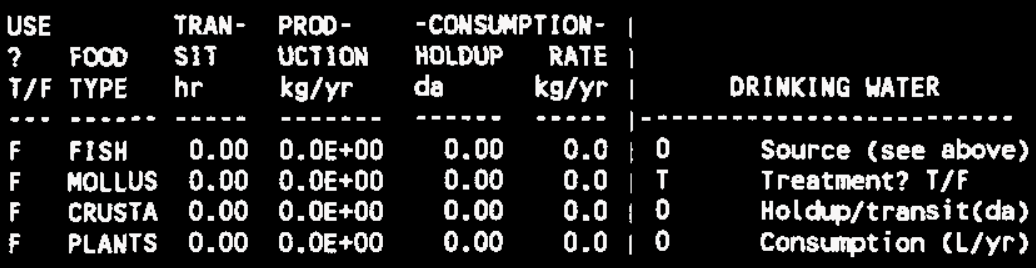

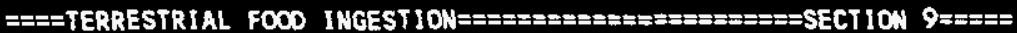

\begin{tabular}{|c|c|c|c|c|c|c|c|c|c|}
\hline$S E$ & & GROW & & IRRIG: & TrON-. & & & $-c$ & \\
\hline$T / F$ & $\begin{array}{l}\text { FOOD } \\
\text { TYPE }\end{array}$ & $\begin{array}{l}\text { TIME } \\
\text { da }\end{array}$ & S & $\begin{array}{l}\text { RATE } \\
\text { in/yr }\end{array}$ & $\begin{array}{l}\text { TIME } \\
\text { mo/yr }\end{array}$ & $\begin{array}{l}\text { YIELD } \\
\mathrm{kg} / \mathrm{m}^{2}\end{array}$ & $\begin{array}{l}\text { UCTION } \\
\mathrm{kg} / \mathrm{yr}\end{array}$ & $\begin{array}{l}\text { HOLD } \\
\text { da }\end{array}$ & $\begin{array}{l}\text { RATE } \\
\mathrm{kg} / \mathrm{yr}\end{array}$ \\
\hline & $F y$ & & 0 & & & & 0 & & \\
\hline & i v & & 0 & & & & & & \\
\hline & FRUIT & & 0 & 0. & & & & 5.0 & \\
\hline & GRAIN & & 0 & 0.0 & 0.0 & 0. & $0.0 E+00$ & 180.0 & 0.0 \\
\hline
\end{tabular}

$===$ ANIMAL PRODUCTION CONSUMPTION=m

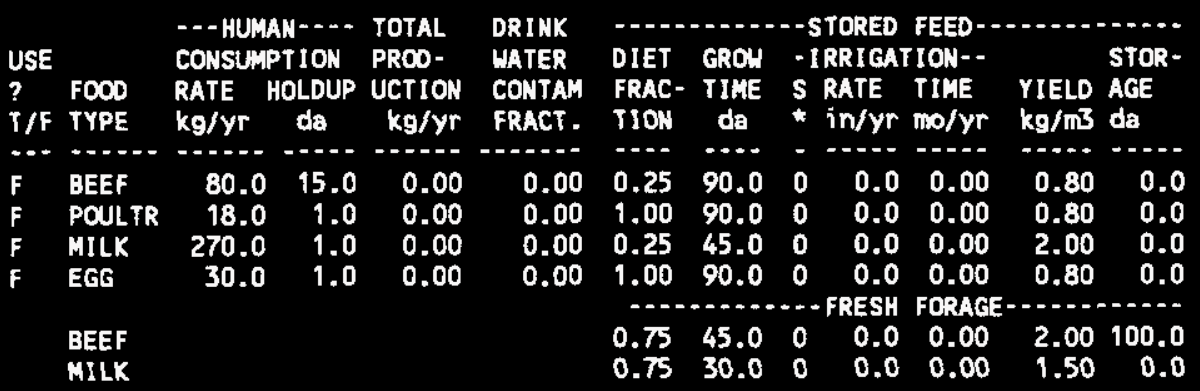




\section{WHC-SD-CP-TI-197 Revision 0}

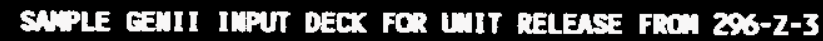

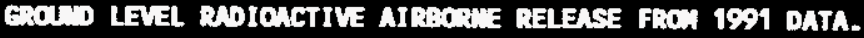

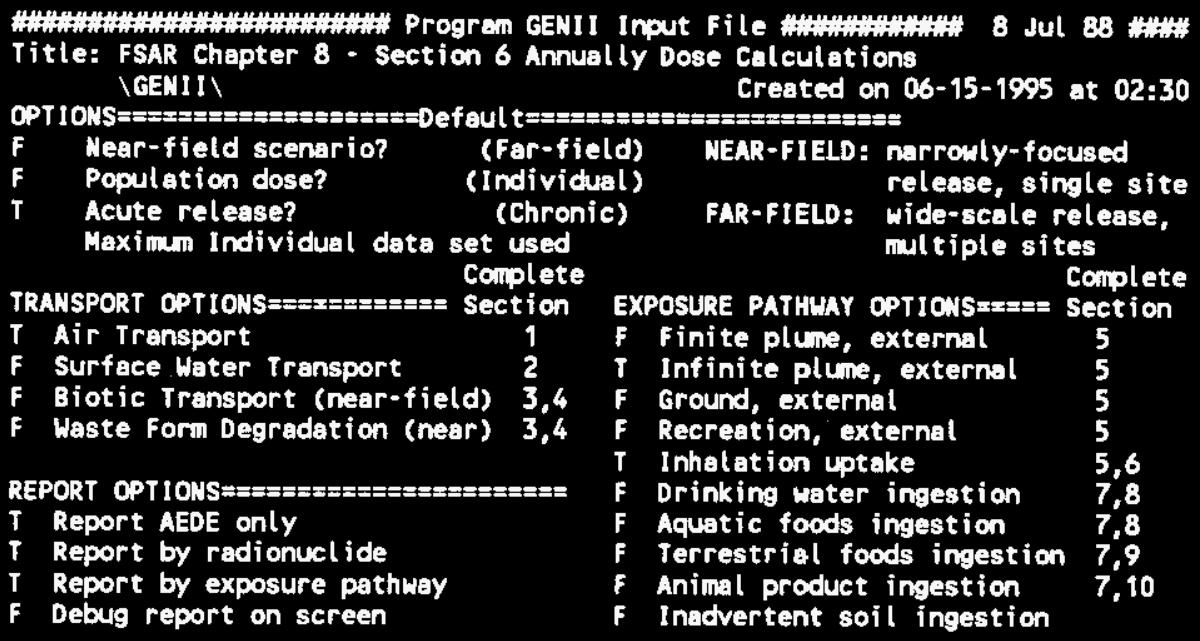

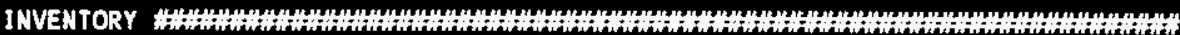

4 Inventory input activity units: (1-pCi 2-uCi 3-mCi 4-Ci 5-Bq)

0 Surface soil source units (1- m2 $\left.2-m_{3} 3-k g\right)$

Equilibriun question goes here

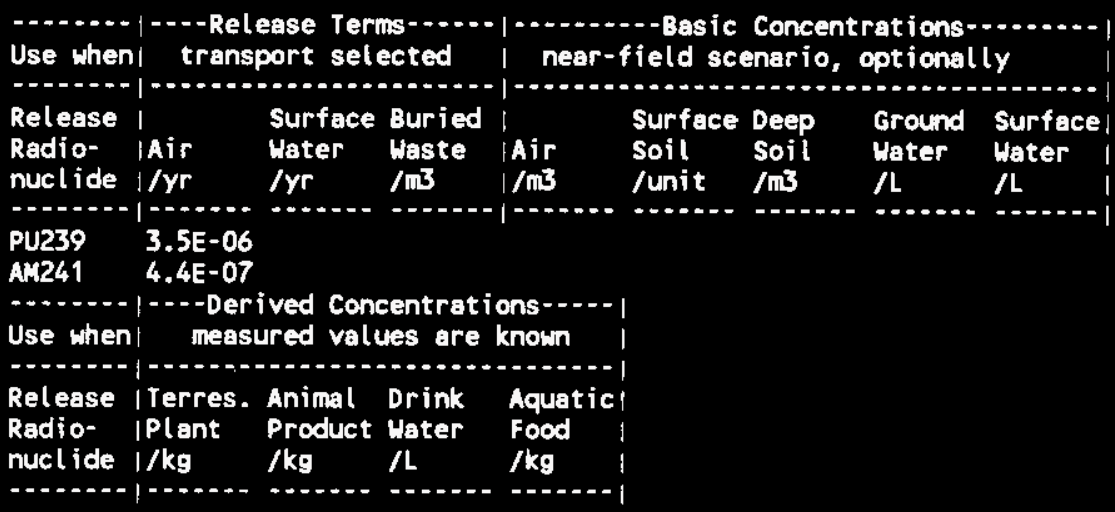

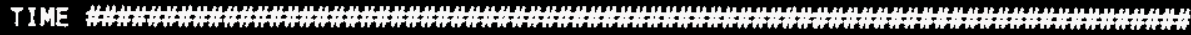

1 Intake ends after (yr)

50 Dose calc. ends after (yr)

0 Release ends after (yr)

0 No. of years of air deposition prior to the intake period

0 No. of years of irrigation water deposition prior to the intake period

FAR-FIELD SCENARIOS (IF POPULATION DOSE) 林)

0 Definition option: 1-Use population grid in file POP.IN 


\section{WHC-SD-CP-TI-197 Revision 0}

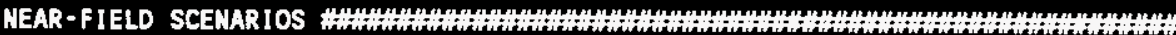

Prior to the beginning of the intake period: (yr)

When was the inventory disposed? (Package degradation starts)

When was LOIC? (Biotic transport starts)

Fraction of roots in upper soil (top $15 \mathrm{~cm}$ )

Fraction of roots in deep soil

Manual redistribution: deep soil/surface soil dilution factor

Source area for external dose modification factor $\left(\mathrm{m}^{2}\right)$

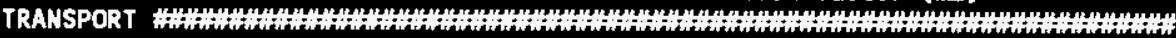

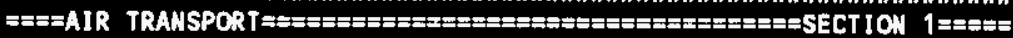
0 -Calculate PM $10 \quad$ Release type (0-3)

1 Option: 1-Use chi/Q or PM value IF stack release (T/F) 2-Select MI dist \& dir $10 \quad$ stack height (m) 3-Specify MI dist \& dir $10 \quad$ stack flou $\left(\mathrm{m}^{3} / \mathrm{sec}\right)$

$\begin{array}{lll}1.0 & \text { Chi/Q or PM value } & 10 \\ 0 & \text { MI sector index }(1=5) & 10\end{array}$

MI distance from release point $(m) 10$

Stack radius $(m)$

Effluent temp. (C)

Building $x$-section ( $(\pi 2)$

Use if data, (T/F) else chi/o grid/0 Building height (m)

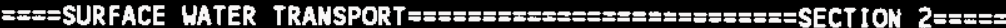
Mixing ratio model: 0 -use value, 1-river, 2 -lake

Mixing ratio, dimensionless

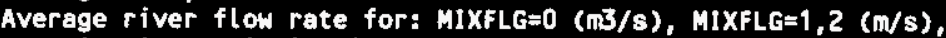

Transit tine to irrigation withdraul (ocation (hr)

If mixing ratio model > 0 :

Rate of effluent discharge to receiving water body $(\mathrm{m} 3 / \mathrm{s})$

Longshore distance from release point to usage location (m)

Offshore distance to the water intake (m)

Average water depth in surface water body $(\mathrm{m})$

Average river width (m), MIXFLG=1 only

Depth of effluent discharge point to surface water (m), lake only

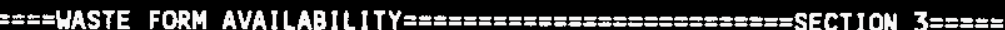
Waste form/package half life, (yr)

Uaste thickness, (m)

Depth of soil overburden, in

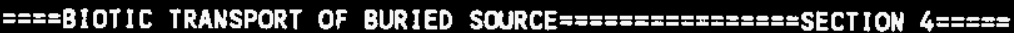
Consider during inventory decay/buildup period (T/F)?

Consider during intake period (T/F)? | 1-Arid non agricultural

Pre-Intake site condition.............. 2-Hunid non agricultural

3-Agricultural

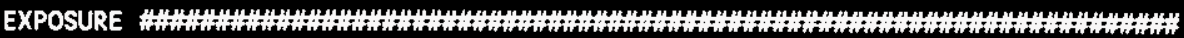

$====E X T E R N A L$ EXPOSURE Exposure tine:

Plune (hr)

Soil contamination (hr)

I Residential irrigation:

Swimming (hr)

Boating (hr)

T

Consider: (T/F)

i 0 Source: 1-ground water

2-surface water

Shoreline activities (hr) i 0 Duration (mo/yr)

Shorel ine type: (1-river, 2-lake, 3-ocean, 4-tidal basin)

Transit time for release to reach aquatic recreation (hr)

Average fraction of time submersed in acute cloud (hr/person hr)

8766.0

$== \pm=$ INHALATIONE=

Hours of exposure to contamination per year

0 -No resus- 1-Use Mass Loading

2-Use Anspaugh model 


\section{WHC-SD-CP-TI-197 Revision 0}

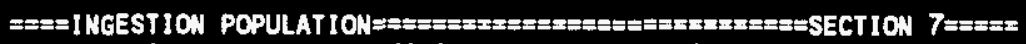
Atmospheric production definition (select option):

0 -Use food-weighted chi/o, (food-sec/mß), enter value on this line 1-Use population-weighted chi/a

2-Use uniform production

3-Use chi/a and production grids (PRCOUCTION will be overridden) Population ingesting aquatic foods, 0 defaults to total (person) Population ingesting drinking water, 0 defaults to total (person) Consider dose from food exported out of region (defaultaf)

Note below: $S^{*}$ or Source: 0 -none, 1-ground water, 2-surface water 3-Derived concentration entered above

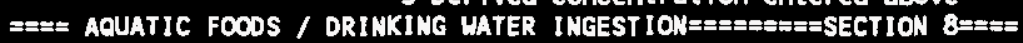

Salt water? (default is fresh)

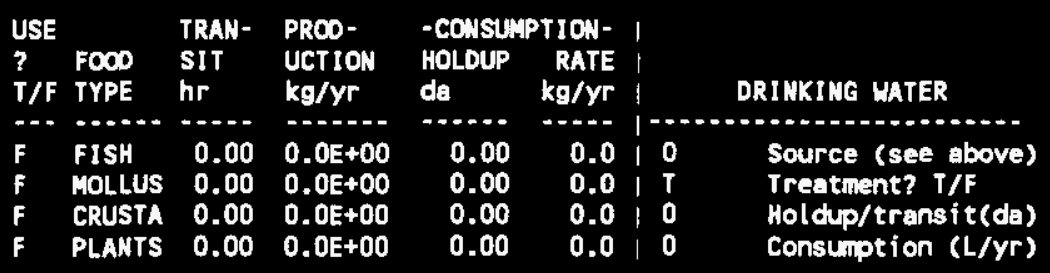

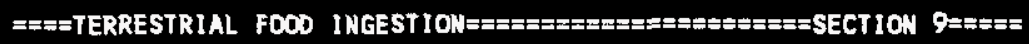

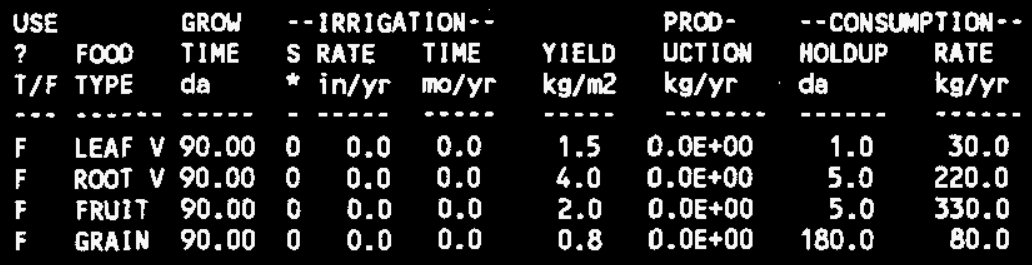

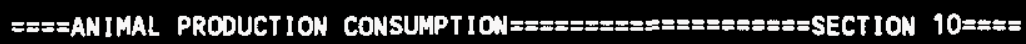

\begin{tabular}{|c|c|c|c|c|c|c|c|c|c|c|c|c|}
\hline \multirow{3}{*}{$\begin{array}{l}\text { USE } \\
? \\
T / F\end{array}$} & \multirow[b]{3}{*}{$\begin{array}{l}\text { FOCD } \\
\text { TYPE }\end{array}$} & \multirow{2}{*}{\multicolumn{2}{|c|}{$\begin{array}{l}- \text { HUMANAN- } \\
\text { CONSUMPTION }\end{array}$}} & \multirow{3}{*}{$\begin{array}{l}\text { TOTAL } \\
\text { PROD- } \\
\text { UCTION } \\
\mathrm{kg} / \mathrm{Yr}\end{array}$} & \multirow{3}{*}{$\begin{array}{l}\text { DRINK } \\
\text { UATER } \\
\text { CONTAM } \\
\text { FRACT. }\end{array}$} & & \multicolumn{2}{|c|}{ TORED } & \multirow{2}{*}{$\begin{array}{l}\text { FEED-- } \\
\text { IOY.- }\end{array}$} & & \multirow[b]{2}{*}{ STOR- } \\
\hline & & & & & & DIET & & -1 & RRIGAT & & & \\
\hline & & $\begin{array}{l}\text { RATE } \\
\mathrm{kg} / \mathrm{yr}\end{array}$ & $\begin{array}{l}\text { HOLDUP } \\
\text { da }\end{array}$ & & & $\begin{array}{l}\text { FRAC- } \\
\text { TIOW }\end{array}$ & $\underset{d a}{\text { TIME }}$ & s & & $\begin{array}{l}\text { TIME } \\
\mathrm{mo} / \mathrm{yr}\end{array}$ & $\begin{array}{l}\text { YIELD } \\
\mathrm{kg} / \mathrm{mB}^{3}\end{array}$ & \\
\hline & & & & & & & & & & & & \\
\hline $\mathbf{F}$ & BEE & 80.0 & 15.0 & 0.0 & 0. & 0.2 & & 0 & 0.0 & 0.00 & & 0.0 \\
\hline & & & & & & & & 0 & & & & \\
\hline & MIL & 270 & 9.0 & & 0. & & & 0 & 0.0 & 0.00 & & \\
\hline & EGG & & 1.0 & D & & & & 0 & 0.0 & 0.00 & & 0. \\
\hline & & & & & & & & & & & & \\
\hline & $\begin{array}{l}\text { BEEF } \\
\text { M1LK }\end{array}$ & & & & & $\begin{array}{l}0.75 \\
0.75\end{array}$ & $\begin{array}{l}45.0 \\
30.0\end{array}$ & $\begin{array}{l}0 \\
0\end{array}$ & $\begin{array}{l}0.0 \\
0.0\end{array}$ & $\begin{array}{l}0.00 \\
0.00\end{array}$ & & $\begin{array}{l}0.0 \\
0.0\end{array}$ \\
\hline
\end{tabular}




\section{WHC-SD-CP-TI-197 Revision 0}

\section{GEII OUTUT DECX FOR ELEVATED UIT RELEASE}

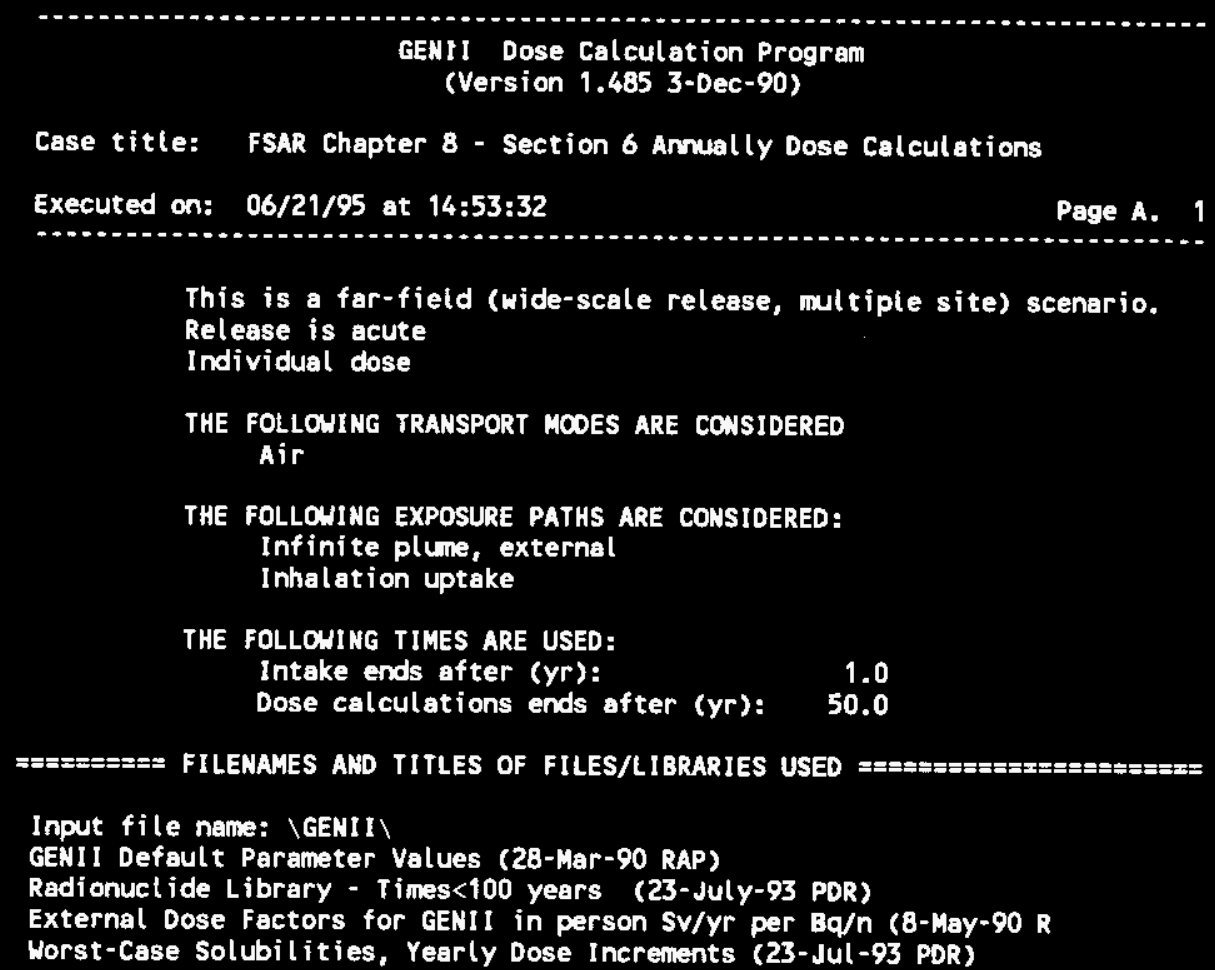

This is a far-field (wide-scale release, multiple site) scenario.

Release is acute

Individual dose

THE FOLLOUING TRANSPORT MODES ARE CONSIDERED

Air

THE FOLLOUING EXPOSURE PATHS ARE CONSIDERED:

Infinite plune, external

Inhalat ion uptake

THE FOLLOUING TIMES ARE USED:

Intake ends after (yr): $\quad 1.0$

Dose calculations ends after (yr): $\quad \mathbf{5 0 . 0}$

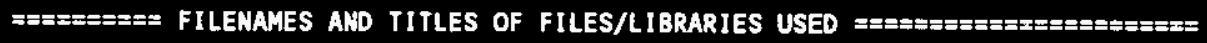

Input file name: \GENII

GENII Default Parameter Values (28-Mar-90 RAP)

Radionuct ide Library - Times<100 years (23-July-93 POR)

External Dose Factors for GENII in person SV/yr per Bq/n (8-May-90 R

Worst-Case Solubilities, Yearly Dose Inerements (23-Jul-93 PDR)

Input prepared by:

Date: 


\section{WHC-SD-CP-TI-197 Revision 0}

Date:

GENII Dose Calculation Program

(Version 1.485 3-Dec-90)

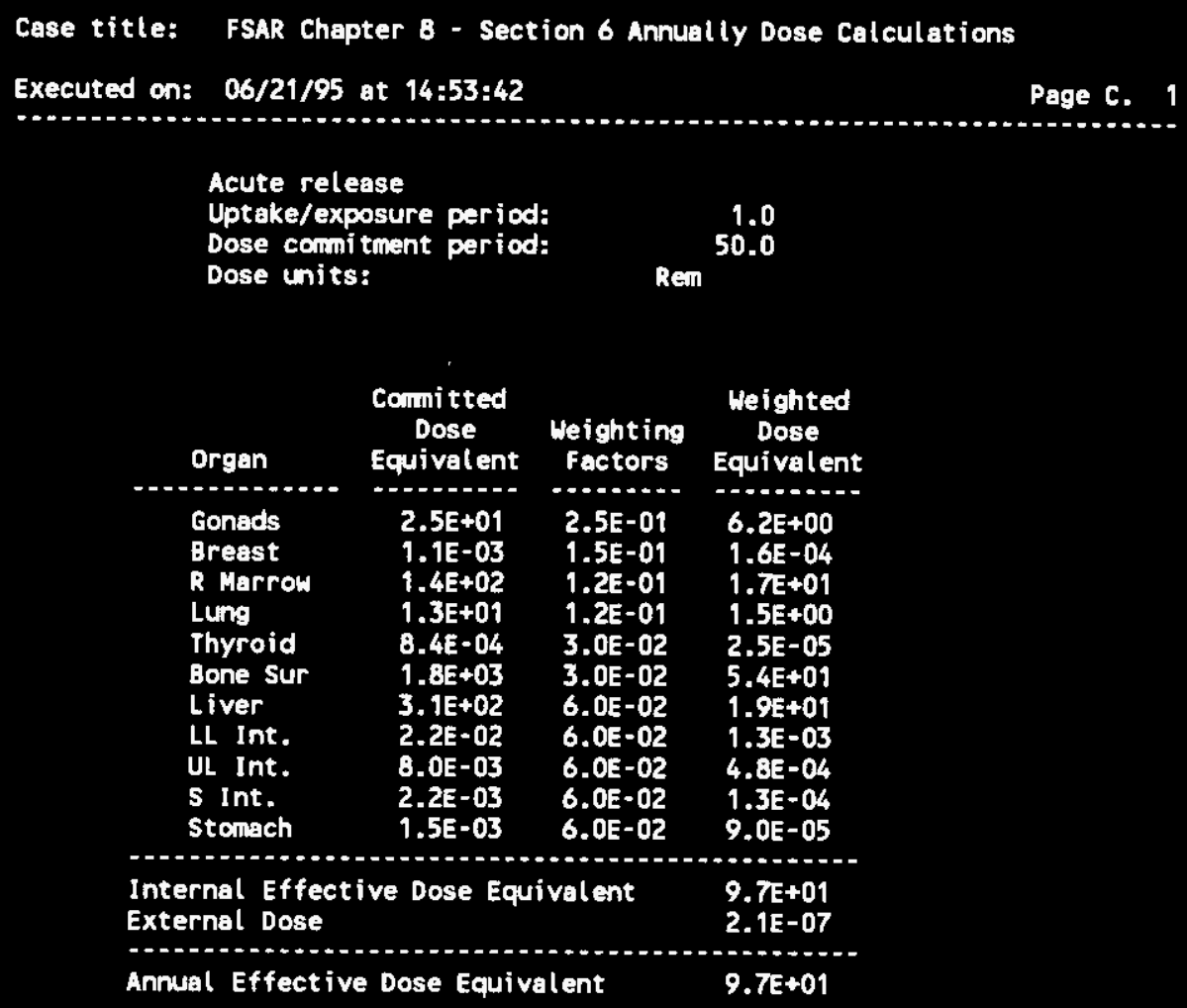

\begin{tabular}{|c|c|}
\hline $\begin{array}{l}\text { Control ling Organ: } \\
\text { Control l ing Pathway: } \\
\text { Controll ling Radi onucl ide: }\end{array}$ & $\begin{array}{l}\text { Bone Sur } \\
\text { Inh } \\
\text { PU239 }\end{array}$ \\
\hline $\begin{array}{l}\text { Total Inhalation EDE: } \\
\text { Total Ingestion EDE: }\end{array}$ & $\begin{array}{l}9.7 E+01 \\
0.0 E+00\end{array}$ \\
\hline
\end{tabular}

GENII Dose Calculation Progran 


\section{WHC-SD-CP-TI-197 Revision 0}

(Version 1.485 3-Dec-90)

Case title: FSAR Chapter 8 - Section 6 Annully Dose Calculations

Executed on: $06 / 21 / 95$ at $14: 53: 42$

Page C. 2

Acute release

Uptake/exposure period:

Dose commitment period:

1.0

Dose units:

50.0

Rem

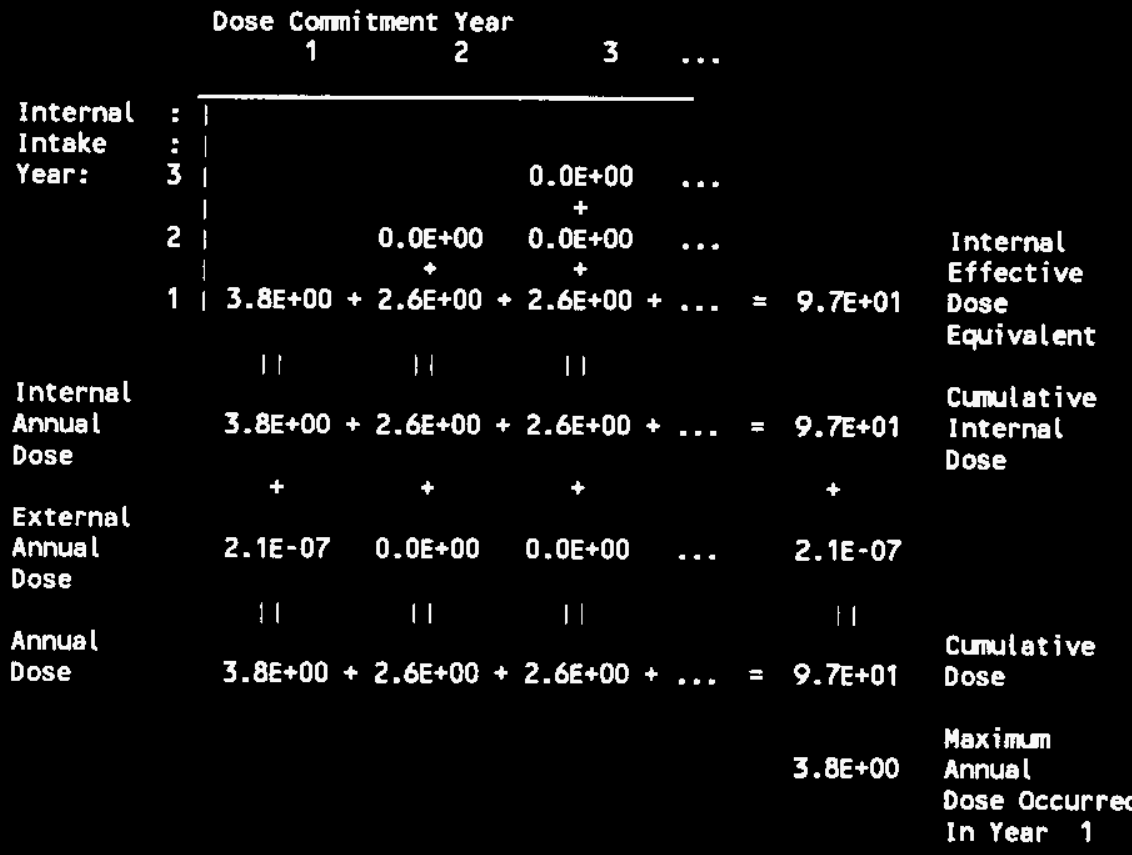

GENII Dose Calculation Program

(Version 1.485 3-Dec-90)

Case title: FSAR Chapter 8 - Section 6 Arnually Dose Calculations

Executed on: $06 / 21 / 95$ at $14: 53: 42$

Page C. 3

Acute release

Uptake/exposure period:

Dose comitment period:

Dose units:

1.0

50.0

Rem

Committed Dose Equivalent by Exposure Pathway 


\section{WHC-SD-CP-TI-197 Revision 0}

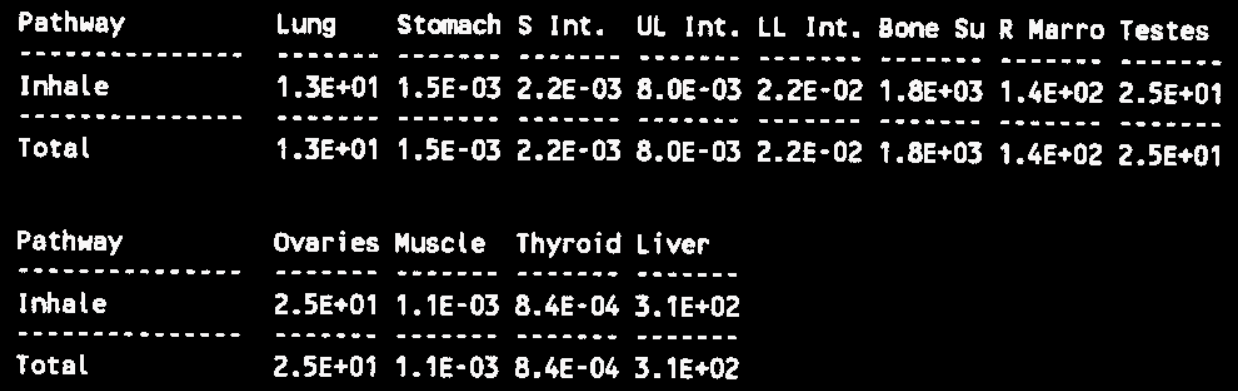

External Dose by Exposure Pathway

\begin{tabular}{ll} 
Pathway & \\
\hline Plume & 2.1E-07 \\
\hline Total & 2.1E-07 \\
\hline
\end{tabular}

GENII Dose Calculation Program

(Version 1.485 3-Dec-90)

Case title: FSAR Chapter 8 - Section 6 Amually Dose Calculations

Executed on: 06/21/95 at 14:53:42

Page C. 4

Acute release

Uptake/exposure period:

Dose conmitment period:

Dose units:

1.0
50.0

Comitted Dose Equivalent by Radionucl ide

\begin{tabular}{lllllllll} 
Radionuclide & Lung & Stonach S Int. UL Int. LL Int. Bone Su R Marro Testes \\
\hdashline PU 241 & $-\cdots .0 E-02$ & $1.5 E-04$ & $1.8 E-04$ & $3.9 E-04$ & $6.5 E-04$ & $1.7 E+02$ & $1.3 E+01$ & $2.6 E+00$ \\
U 237 & $0.0 E+00$ & $0.0 E+00$ & $0.0 E+00$ & $0.0 E+00$ & $0.0 E+00$ & $0.0 E+00$ & $0.0 E+00$ & $0.0 E+00$ \\
AM 241 & $2.1 E+00$ & $4.0 E-04$ & $5.0 E-04$ & $1.5 E-03$ & $3.7 E-03$ & $2.5 E+02$ & $2.0 E+01$ & $3.5 E+00$ \\
PU 239 & $1.1 E+01$ & $9.4 E-04$ & $1.5 E-03$ & $6.1 E-03$ & $1.8 E-02$ & $1.4 E+03$ & $1.1 E+02$ & $1.9 E+01$ \\
\hdashline & $1.3 E+01$ & $1.5 E-03$ & $2.2 E-03$ & $8.0 E-03$ & $2.2 E-02$ & $1.8 E+03$ & $1.4 E+02$ & $2.5 E+01$
\end{tabular}

Radionucl ide

Ovaries Muscle Thyroid Liver

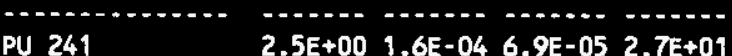

U $237 \quad 0.0 E+00 \quad 0.0 E+00 \quad 0.0 E+00 \quad 0.0 E+00$

AM $241 \quad 3.5 E+003.4 E-04 \quad 1.9 E-04 \quad 4.5 E+01$

PU $2391.9 E+015.8 E-045.7 E-042.4 E+02$

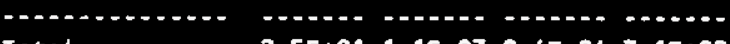

Total 2.5E+01 1.1E-03 8.4E-04 3.1E+02 


\section{WHC-SD-CP-TI-197 Revision 0}

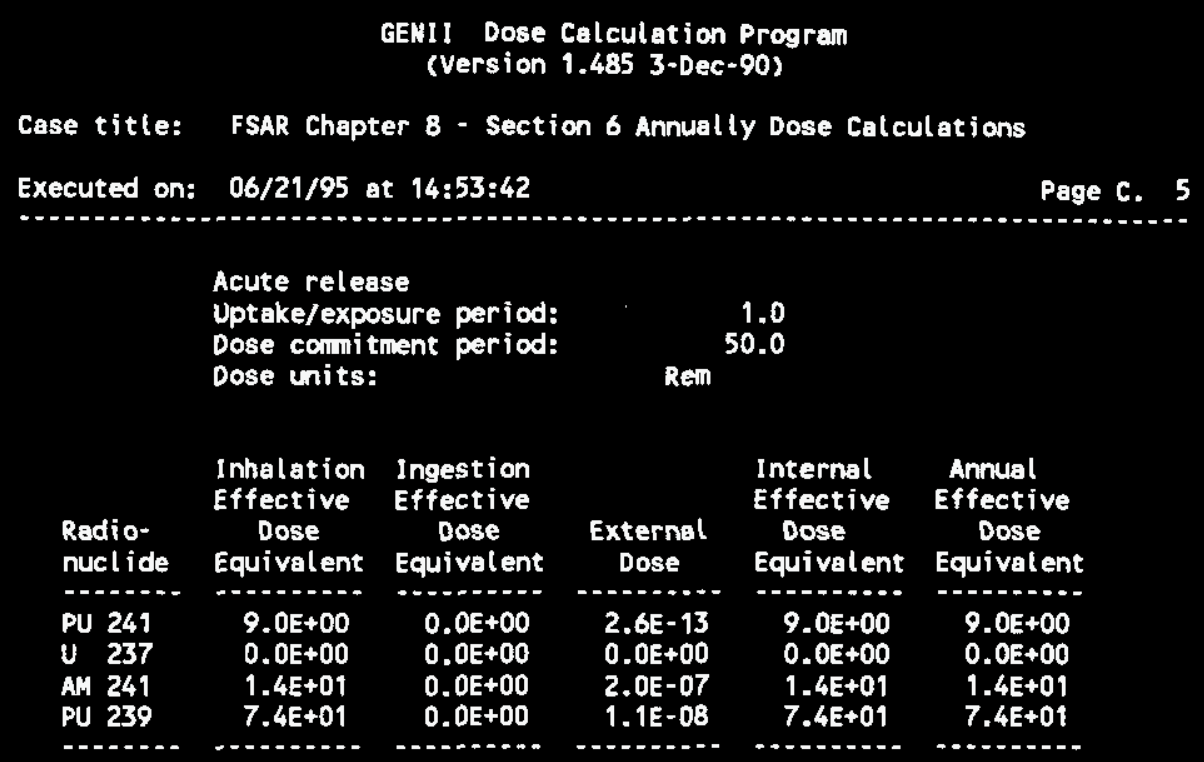




\section{SAMPLE UNIT RELEASE FOR GROUND LEVEL RELEASE}

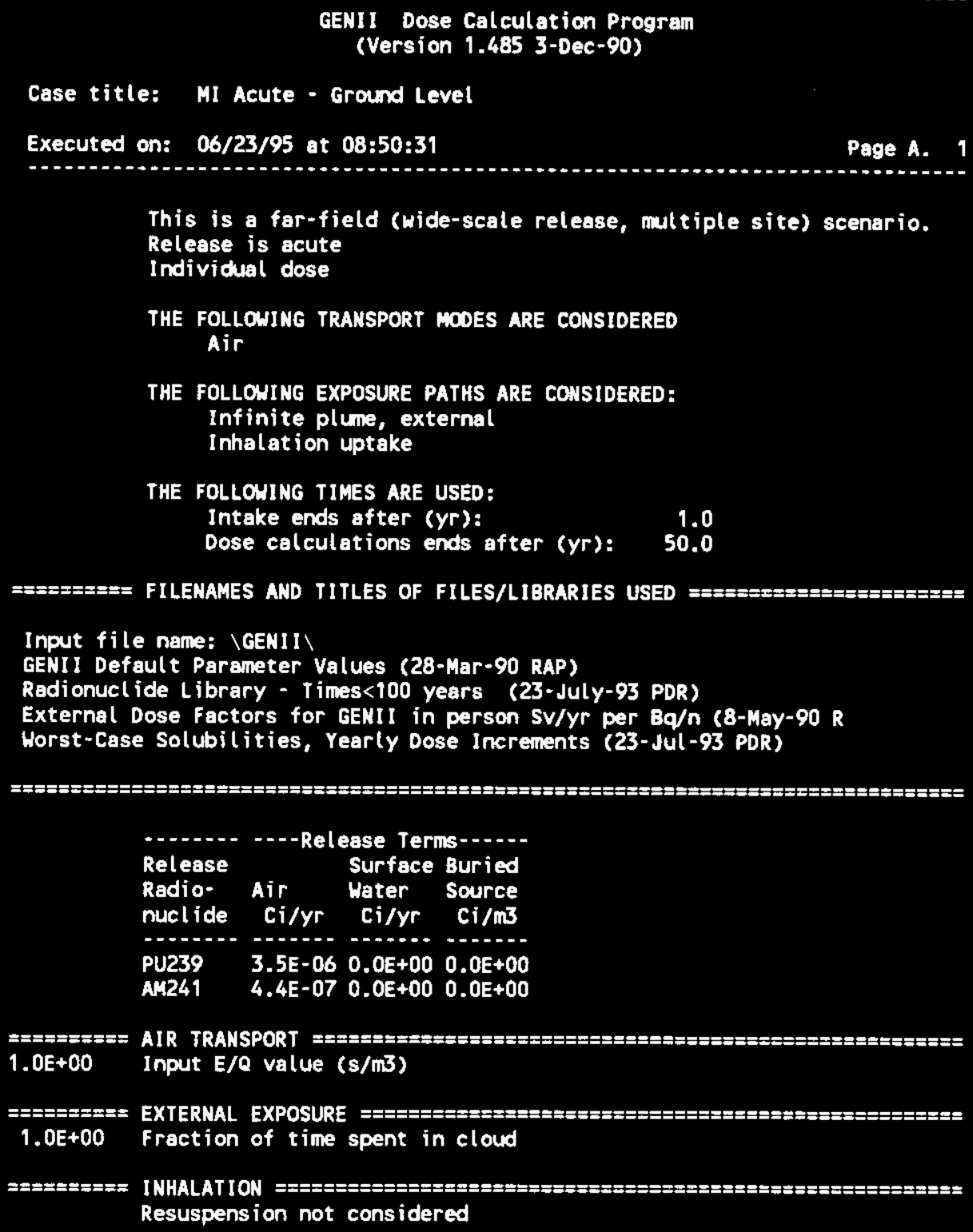

This is a far-field (wide-scale release, muttiple site) scenario. Release is acute

THE FOLLOUING TIMES ARE USED: Intake ends after (yr): $\quad 1.0$ Dose calculations ends after $(y r): \quad 50.0$

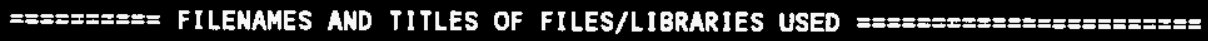

Input file nane: \GENII

GENII Default Paraneter Values (28-Mar-90 RAP)

Radionucl ide Library - Times<100 years (23-July-93 PDR)

External Dose Factors for GENII in person SV/yr per Bq/n (8-May-90 R

Worst-Case Solubilities, Yearly Dose Increnents (23-Jul-93 PDR)

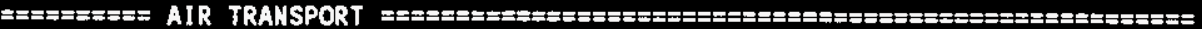

Input prepared by:

Date:

Input checked by:

Date: 


\section{WHC-SD-CP-TI-197 Revision 0}

GENII Dose Calculation Progran

(Version 1.485 3-Dec-90)

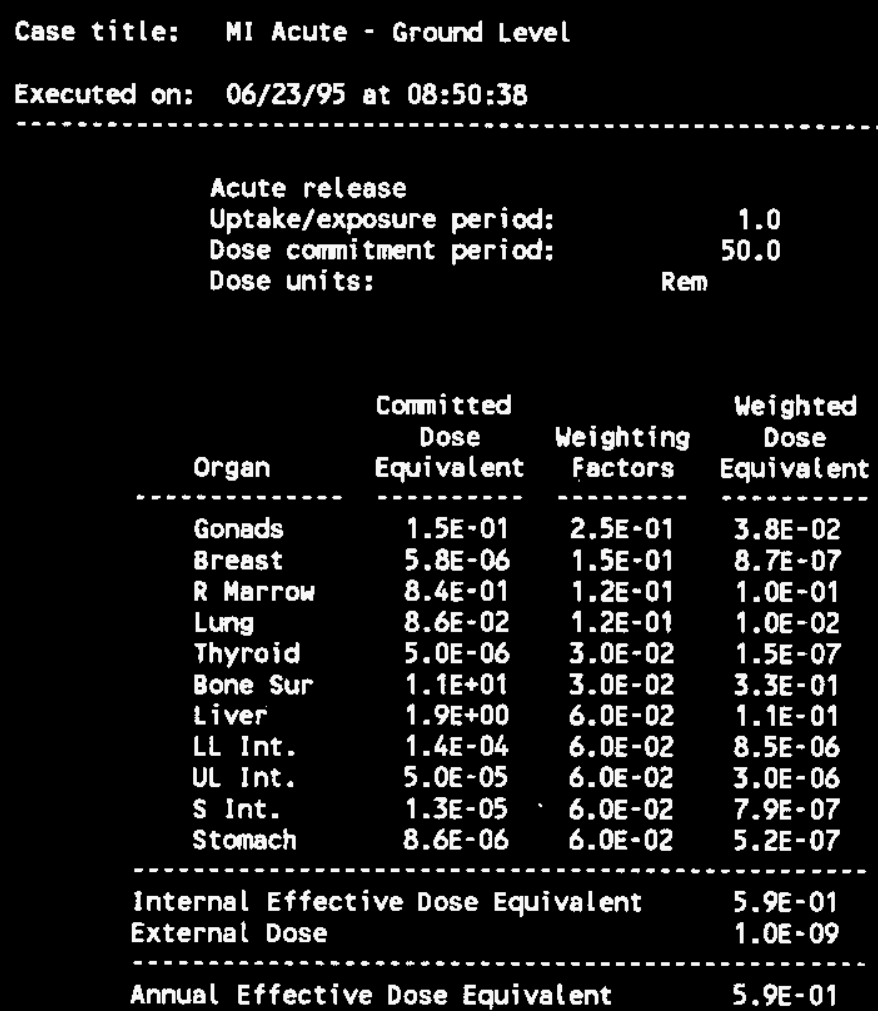

Page C. 1

Acute release

Aptake/exposure period:

Dose comitment period:

$5.9 E-01$

\begin{tabular}{ll} 
Controll ing Organ: & $\begin{array}{l}\text { Bone sur } \\
\text { Inh } \\
\text { Control ling Pathway: } \\
\text { Control ling Radionucl ide: }\end{array}$ \\
\hline Total Inhalation EDE: & $5.9 E-01$ \\
Total Ingestion EDE: & $0.0 E+00$
\end{tabular}

GENI Dose Calculation Program (Version 1.485 3-Dec-90)

Case title: MI Acute - Ground Level

Executed on: 06/23/95 at 08:50:38

Page C. 2 


\section{WHC-SD-CP-TI-197 Revision 0}

Acute release
Uptake/exposure period:
Dose conmitinent period:

Dose units:
1.0

Rem

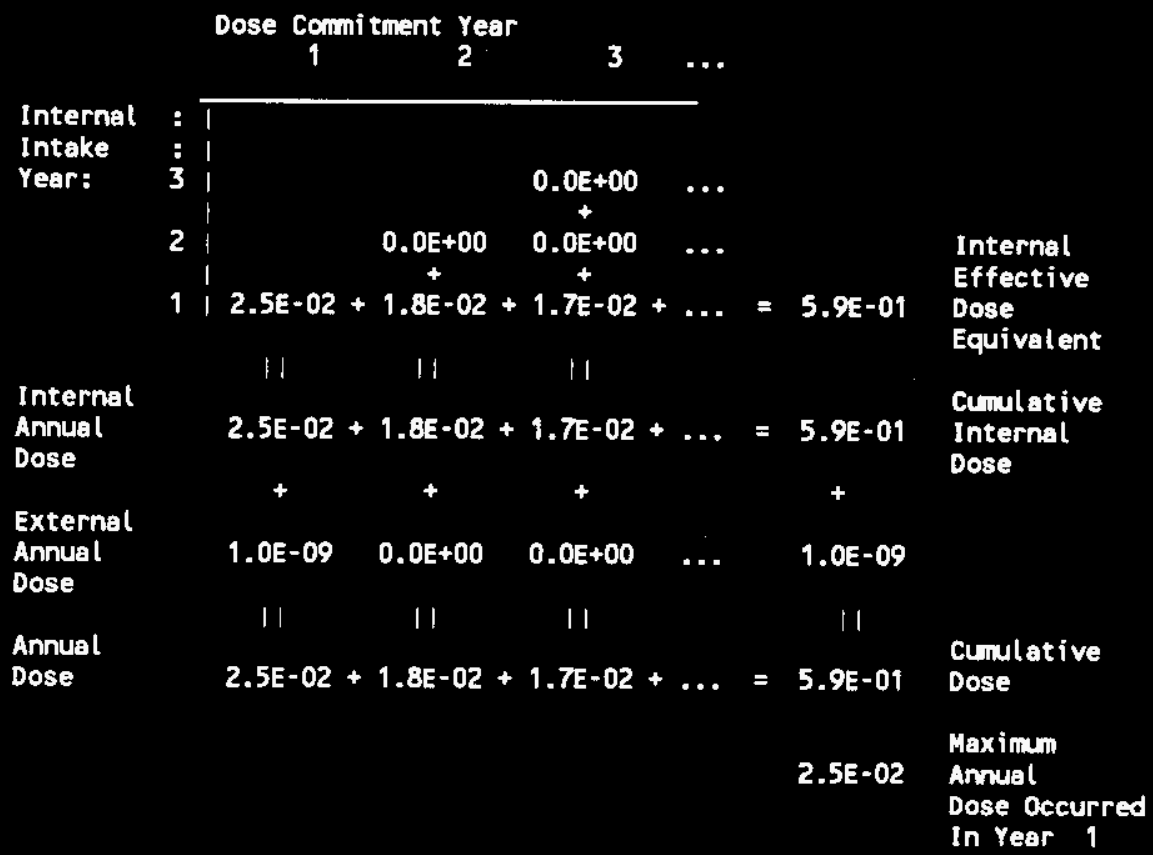

Conmitted Dose Equivalent by Exposure Pathway

Pathway

Lung Stomach S Int. UL Int. LL Int. Bone Su R Marro Testes

Prenten

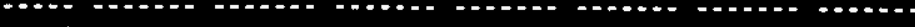

Inhale 8.6E-02 8.6E-06 1.3E-05 5.0E-05 1.4E-04 1.1E+01 8.4E-01 1.5E-01 Total 8.6E-02 8.6E-06 1.3E-05 5.0E-05 1.4E-04 1.1E+01 8.4E-01 1.5E-01 


\section{WHC-SD-CP-TI-197 Revision 0}

\begin{tabular}{lllll} 
Pathway & Ovaries Muscle & Thyroid Liver \\
\hline Inhale & $1.5 E-01$ & $5.8 E-06$ & $5.0 E-06$ & $1.9 E+00$ \\
\hdashline Total & $1.5 E-01$ & $5.8 E-06$ & $5.0 E-06$ & $1.9 E+00$
\end{tabular}

External Dose by Exposure Pathway

\begin{tabular}{ll} 
Pathway & \\
\hline Plume & $1.0 E-09$ \\
\hline Total & $1.0 \mathrm{E}-09$ \\
\hline
\end{tabular}

GENII Dose Calculation Progran

(Version 1.485 3-Dec-90)

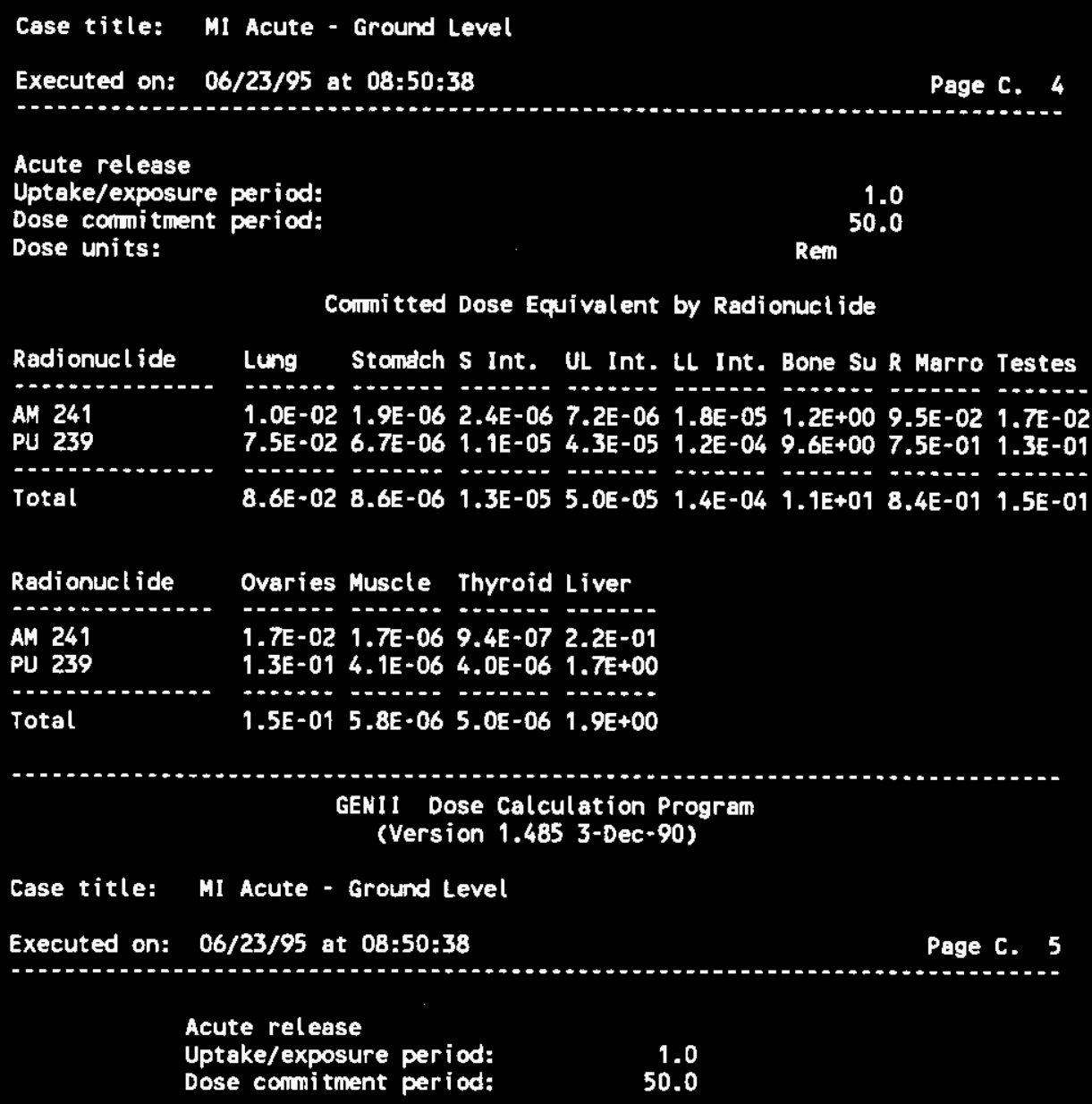




\section{WHC-SD-CP-TI-197 Revision 0}

\begin{tabular}{|c|c|c|c|c|c|}
\hline & Dose units: & & Rem & & \\
\hline $\begin{array}{l}\text { Radio- } \\
\text { muct ide } \\
\text { AN } 241 \\
\text { PU } 239\end{array}$ & $\begin{array}{c}\text { Inhalation } \\
\text { Effective } \\
\text { Dose } \\
\text { Equivalent } \\
6.6 E-02 \\
5.2 E-01\end{array}$ & $\begin{array}{c}\begin{array}{c}\text { Ingestion } \\
\text { Effective } \\
\text { Dose }\end{array} \\
\text { Equivalent } \\
0.0 E+00 \\
0.0 E+00\end{array}$ & $\begin{array}{c}\text { External } \\
\text { Dose } \\
9.7 E-10 \\
7.4 E-11\end{array}$ & $\begin{array}{l}\text { Internal } \\
\text { Effective } \\
\text { Dose } \\
\text { Equivalent } \\
6.6 E-02 \\
5.2 E-01\end{array}$ & $\begin{array}{c}\begin{array}{c}\text { Annus } \\
\text { Effect ive } \\
\text { Dose } \\
\text { Equivalent } \\
6.6 E-02 \\
5.2 E-01\end{array}\end{array}$ \\
\hline
\end{tabular}

GXO OUTPUT DECKS 


\section{WHC-SD-CP-TI-197 Revision 0}

GXa Version 4.0

Decenber 19, 1994

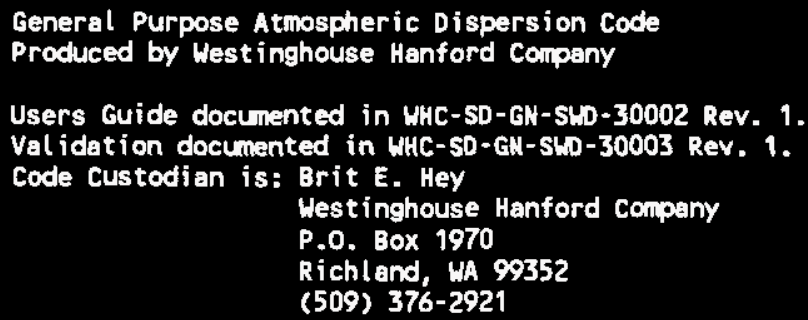

ONSITE AND OFFSITE 99.5 PERCENTILE X/Q ACUTE GROUND LEVEL RELEASE c GXa Version 4.0 Input File 


\section{WHC-SD-CP-TI-197 Revision 0}

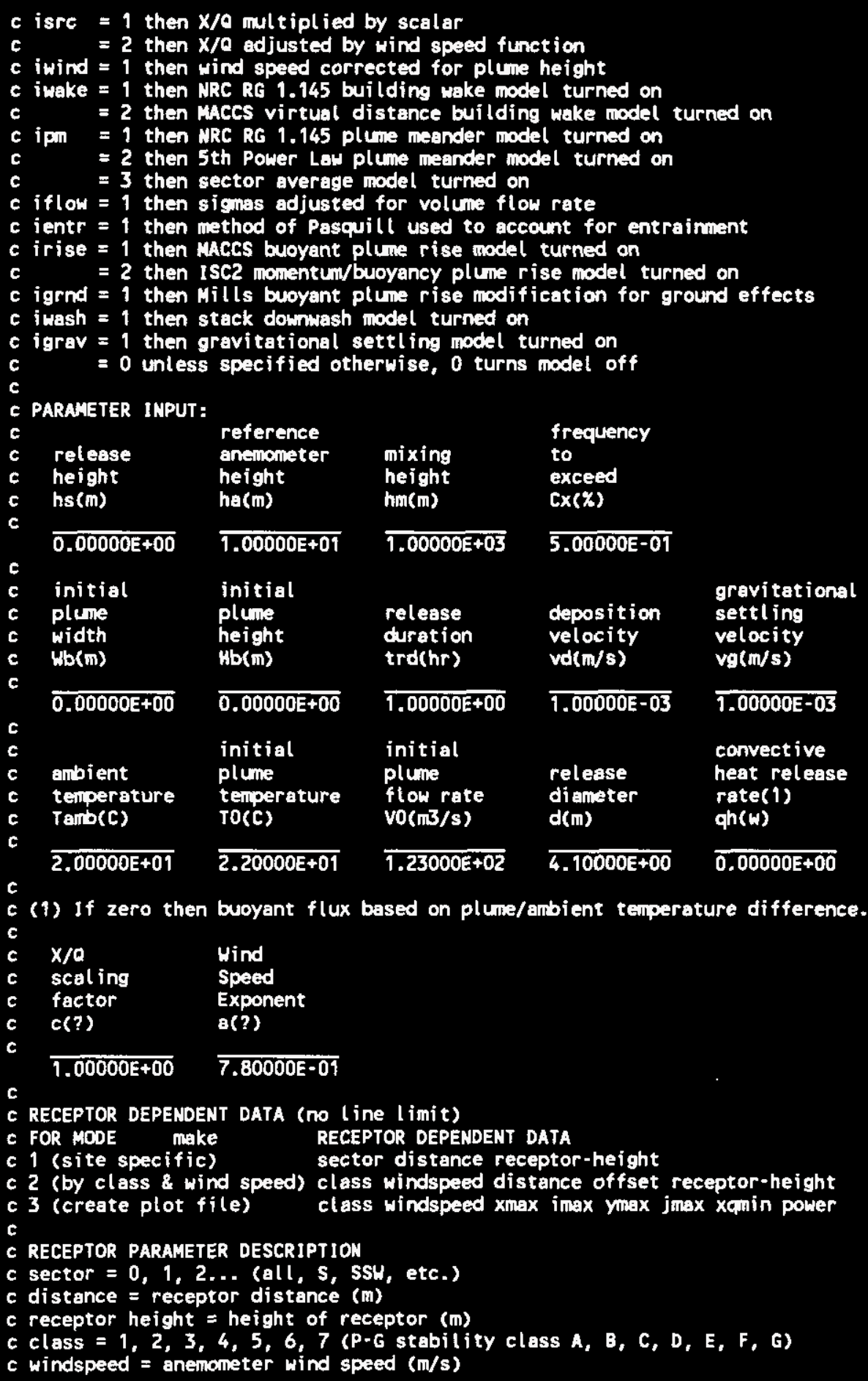




\section{WHC-SD-CP-TI-197 Revision 0}

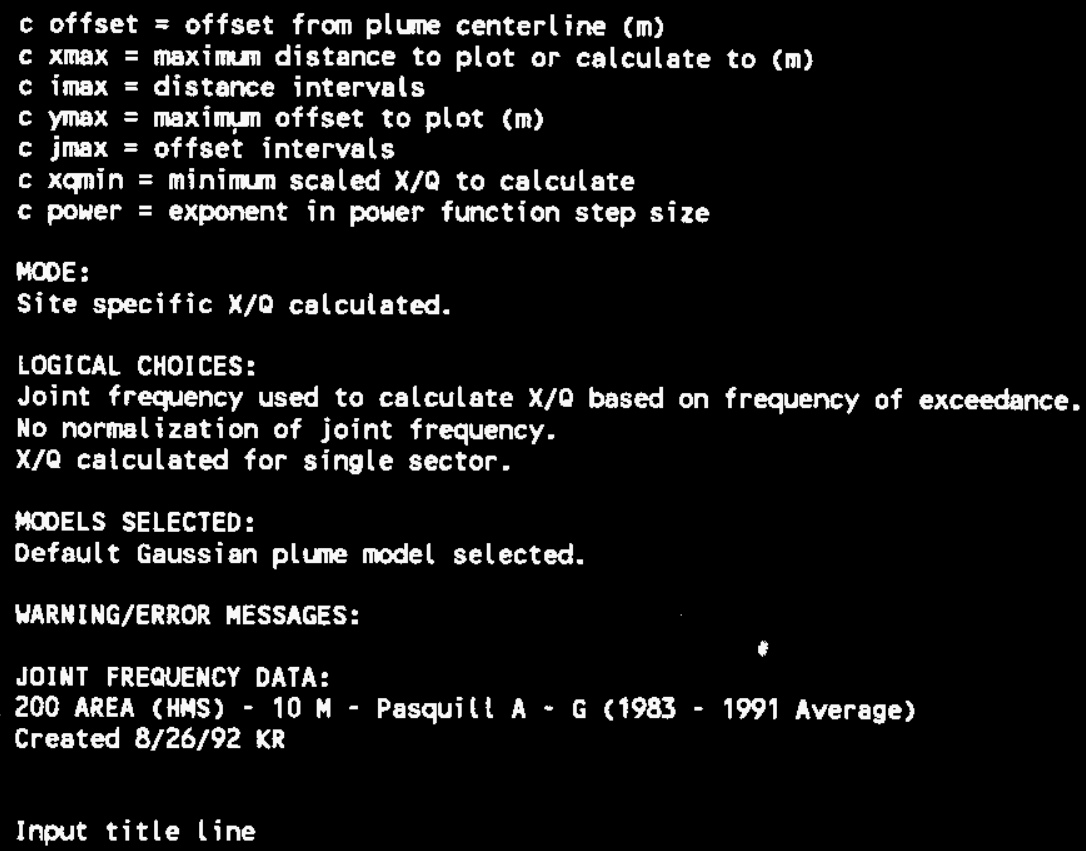

\begin{tabular}{|c|c|c|c|c|c|c|c|c|}
\hline SECTOR & $\begin{array}{l}\text { DISTANCE } \\
\text { (m) }\end{array}$ & $\begin{array}{l}\text { RECEPT } \\
\text { HEIGHT } \\
\text { (m) }\end{array}$ & $\begin{array}{l}\text { SECT. } \\
\text { FREQ. } \\
\text { (X) }\end{array}$ & POPULATION & $\begin{array}{l}\text { TOTAL } \\
\text { POPULATION } \\
\text { SCALED } \\
X / \theta \\
(\mathrm{s} / \mathrm{m} 3)\end{array}$ & $\begin{array}{l}\text { AVERAGE } \\
\text { INDIVIDUAL } \\
\text { SCALED } \\
\text { X/a } \\
(\mathrm{s} / \mathrm{m} 3)\end{array}$ & $\begin{array}{l}\text { ATM. } \\
\text { STAB. } \\
\text { CLASS }\end{array}$ & $\begin{array}{l}\text { WIND } \\
\text { SPEED } \\
(m / s)\end{array}$ \\
\hline $\begin{array}{l}\text { UNH } \\
\text { NE } \\
\text { ENE } \\
\text { ESE } \\
\text { SSE } \\
\text { S } \\
\text { SSW } \\
\text { SW } \\
\text { WSW } \\
W \\
\text { WNH } \\
\text { WH } \\
\text { WWH } \\
\text { N } \\
\text { NNE } \\
\text { NE } \\
\text { ENE } \\
\text { E } \\
\text { ESE } \\
\text { SE } \\
\text { SSE }\end{array}$ & $\begin{array}{r}550 \\
1540 \\
750 \\
1050 \\
930 \\
15000 \\
15400 \\
16100 \\
13200 \\
12500 \\
13200 \\
16500 \\
17400 \\
19200 \\
26000 \\
28900 \\
25600 \\
25200 \\
30000 \\
25200 \\
22900\end{array}$ & $\begin{array}{l} \\
0 \\
0 \\
0 \\
0 \\
0 \\
0 \\
0 \\
0 \\
0 \\
0 \\
0 \\
0 \\
0 \\
0 \\
0 \\
0 \\
0 \\
0 \\
0 \\
0\end{array}$ & $\begin{array}{r}3.98 \\
3.90 \\
6.17 \\
18.80 \\
4.78 \\
6.30 \\
4.53 \\
2.93 \\
2.72 \\
4.80 \\
3.98 \\
4.72 \\
4.58 \\
4.36 \\
2.49 \\
3.90 \\
6.17 \\
14.05 \\
18.80 \\
10.83 \\
4.78\end{array}$ & $\begin{array}{l}1 \\
1 \\
1 \\
1 \\
1 \\
1 \\
1 \\
1 \\
1 \\
1 \\
1\end{array}$ & $\begin{array}{l}8.72 E-04 \\
1.59 E-04 \\
8.22 E-04 \\
6.03 E-04 \\
3.59 E-04 \\
6.71 E-06 \\
4.10 E-06 \\
4.97 E-06 \\
6.40 E-06 \\
1.62 E-05 \\
8.49 E-06 \\
1.18 E-05 \\
1.11 E-05 \\
1.05 E-05 \\
3.15 E-06 \\
2.98 E-06 \\
6.02 E-06 \\
8.21 E-06 \\
6.34 E-06 \\
7.44 E-06 \\
4.03 E-06\end{array}$ & $\begin{array}{l}8.72 E-04 \\
1.59 E-04 \\
8.22 E-04 \\
6.03 E-04 \\
3.59 E-04 \\
6.71 E-06 \\
4.10 E-06 \\
4.97 E-06 \\
6.40 E-06 \\
1.62 E-05 \\
8.49 E-06 \\
1.18 E-05 \\
1.11 E-05 \\
1.05 E-05 \\
3.15 E-06 \\
2.98 E-06 \\
6.02 E-06 \\
8.21 E-06 \\
6.34 E-06 \\
7.44 E-06 \\
4.03 E-06\end{array}$ & $\begin{array}{l}G \\
E \\
G \\
F \\
G \\
E \\
F \\
F \\
F \\
G \\
G \\
G \\
G \\
G \\
E \\
E \\
G \\
F \\
F \\
G \\
G\end{array}$ & $\begin{array}{l}4.70 \\
0.89 \\
2.65 \\
0.89 \\
4.70 \\
0.89 \\
2.65 \\
2.65 \\
2.65 \\
2.65 \\
4.70 \\
2.65 \\
2.65 \\
2.65 \\
0.89 \\
0.89 \\
2.65 \\
0.89 \\
0.89 \\
2.65 \\
4.70\end{array}$ \\
\hline
\end{tabular}


WHC-SD-CP-TI-197 Revision 0

QXe Version 4.0

Decenber 19, 1994

General Purpose Atmospheric Dispersion Code

Produced by Mestinghouse Hanford Company

Users Guide documented in UHC-SD-GN-SWD-30002 Rev. 1.

Validation documented in WHC-SD-GN-SWD-30003 ReV. 1.

Code Custodian is: Brit E. Hey

West inghouse Hanford Company

P.O. Box 1970

Richland, WA 99352

(509) 376-2921

Run Date $=08 / 25 / 95$

Run Time $=13: 34: 05.73$

INPUT ECHO:

Onsite $\&$ Offsite $99.5 \times$ Acute stack Level Release

c GXQ Version 4.0 Input File

c mode

1

$c$

c MODE CHOICE:

$c$ mode $=1$ then $X / Q$ based on Hanford site specific meteorology

$c$ mode $=2$ then $X / Q$ based on atmospheric stability class and wind speed

$c$ mode $=3$ then $X / Q$ plot file is created

c

c LOGICAL CHOICES:

c ifox inorm icdf ichk isite ipop

$T \quad F \quad F \quad F \quad F \quad F$

$c$ ifox $=t$ then joint frequency used to compute frequency to exceed $X / Q$

$c \quad=f$ then joint frequency used to compute annual average $x / 0$

$c$ inorm $=t$ then joint frequency data is normalized (as in GENII)

c $\quad=f$ then joint frequency data is un-normal ized

$c$ icdf $=t$ then cumulative distribution file created (CDF.OUT)

$c \quad=f$ then no cumulative distribution file created

$c$ ichk $=t$ then $X / a$ parameter print option turned on

$c \quad=f$ then no paraneter print

$c$ isite $=t$ then $X / Q$ based on joint frequency data for all 16 sectors

$c=f$ then $X / Q$ based on joint frequency data of individual sectors

$c$ ipop $=t$ then $X / 0$ is population weighted

$c \quad=f$ then no population weighting

C $X / O$ AND WIND SPEED ADJUSTMENT MODELS:

$c$ ipuff idep isre iwind

$\begin{array}{llll}0 & 0 & 0 & 1\end{array}$

c. DIFFUSION COEFFICIENT ADJUSTMENT MOOELS:

c iwake ipn iflow ientr

$\begin{array}{llll}0 & 3 & 1 & 0\end{array}$

c EFFECTIVE RELEASE HEIGHT ADJUSTMENT MODELS:

c (irise igrnd) iwash igrav

$$
20010
$$

$c$ ipuff $=1$ then $x / 0$ calculated using puff model

$c \quad=0$ then $X / Q$ calculated using default continuous plume model 


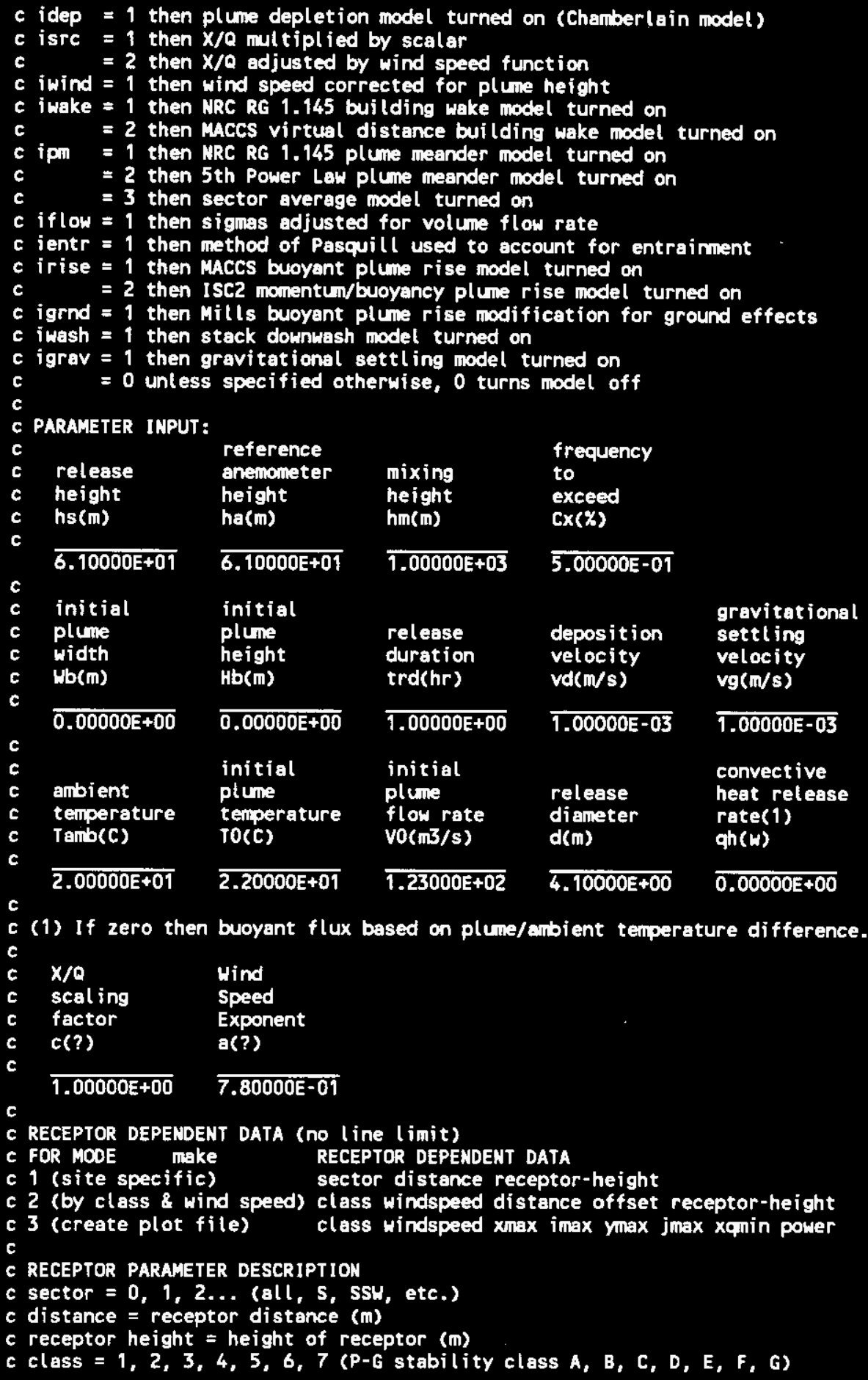


c windspeed = anenometer wind speed $(\mathrm{m} / \mathrm{s})$

c offset $=$ offset from plume centerline $(m)$

c $x_{\text {max }}=$ maximun distance to plot or calculate to (m)

c $i_{\max }=$ distance intervals

c ymax $=$ maximum offset to plot $(m)$

c $\mathrm{jmax}=$ offset intervals

c $x$ minin $=$ minimm scaled $x / 0$ to calculate

$c$ power $=$ exponent in power function step size

MOOE:

Site specific $X / Q$ calculated.

LOGICAL CHOICES:

Joint frequency used to calculate $X / Q$ based on frequency of exceedance. No normal ization of joint frequency.

$X / a$ calculated for single sector.

MODELS SELECTED:

Flou adjustment model.

Sector average model selected.

ISC2 monentun/buoyancy plume model based on temperature difference.

Stack downwash model selected.

Default Gaussian plume model selected.

uind velocity corrected for average plume height.

MARNING/ERROR MESSAGES:

UARNING $\$ 10-x / 0$ may be underestimated for plune rise scenario without accounting for entrainment.

JOINT FREQUENCY DATA:

200 AREA (HMS) - 61 M - Pasquill A - G (1983 - 1991 Average)

Created 8/26/92 KR

Onsite \& Offsite $99.5 \times$ Acute Ground Level Release

\begin{tabular}{|c|c|c|c|c|c|c|c|c|}
\hline SECTOR & $\begin{array}{l}\text { DISTANCE } \\
\text { (m) }\end{array}$ & $\begin{array}{l}\text { RECEPT } \\
\text { HEIGHT } \\
\text { (m) }\end{array}$ & $\begin{array}{l}\text { SECT. } \\
\text { FREQ. } \\
(x)\end{array}$ & POPULATION & $\begin{array}{c}\text { TOTAL } \\
\text { POPULATIOW } \\
\text { SCALED } \\
x / a \\
\left(s / \mathrm{mb}^{3}\right)\end{array}$ & $\begin{array}{c}\text { AVERAGE } \\
\text { INDIVIDUAL } \\
\text { SCALED } \\
X / Q \\
(\mathrm{~s} / \mathrm{mB})\end{array}$ & $\begin{array}{l}\text { ATM. } \\
\text { STAB. } \\
\text { CLASS }\end{array}$ & $\begin{array}{l}\text { UIND } \\
\text { SPEED } \\
(\mathrm{m} / \mathrm{s})\end{array}$ \\
\hline $\begin{array}{l}\text { UNH } \\
\text { NE } \\
\text { ENE } \\
\text { ESE } \\
\text { SSE } \\
\text { S } \\
\text { SSH } \\
\text { SH } \\
\text { WSH } \\
\text { W } \\
\text { WNH } \\
\text { NH } \\
\text { NNH } \\
N \\
\text { NNE } \\
\text { NE } \\
\text { ENE } \\
\text { E }\end{array}$ & $\begin{array}{r}630 \\
1580 \\
830 \\
1060 \\
930 \\
15000 \\
15400 \\
16100 \\
13200 \\
12500 \\
13200 \\
16500 \\
17400 \\
19200 \\
26000 \\
28900 \\
25600 \\
25200\end{array}$ & $\begin{array}{l}0 \\
0 \\
0 \\
0 \\
0 \\
0 \\
0 \\
0 \\
0 \\
0 \\
0 \\
0 \\
0 \\
0 \\
0 \\
0 \\
0 \\
0\end{array}$ & $\begin{array}{r}3.80 \\
3.95 \\
6.33 \\
19.39 \\
4.75 \\
6.11 \\
4.33 \\
2.79 \\
2.54 \\
4.56 \\
3.80 \\
4.58 \\
4.51 \\
4.31 \\
2.48 \\
3.95 \\
6.33 \\
14.39\end{array}$ & $\begin{array}{l}1 \\
1 \\
1 \\
1 \\
1 \\
1 \\
1 \\
1 \\
1 \\
1 \\
1 \\
1 \\
1 \\
1 \\
1 \\
1 \\
1 \\
1\end{array}$ & $\begin{array}{l}5.57 E-06 \\
1.03 E-06 \\
2.16 E-06 \\
2.52 E-06 \\
3.35 E-06 \\
5.28 E-07 \\
4.32 E-07 \\
4.10 E-07 \\
5.10 E-07 \\
6.24 E-07 \\
5.85 E-07 \\
5.10 E-07 \\
5.12 E-07 \\
4.82 E-07 \\
2.01 E-07 \\
1.95 E-07 \\
2.36 E-07 \\
4.35 E-07\end{array}$ & $\begin{array}{l}5.57 E-06 \\
1.03 E-06 \\
.2 .16 E-06 \\
2.52 E-06 \\
3.35 E-06 \\
5.28 E-07 \\
4.32 E-07 \\
4.10 E-07 \\
5.10 E-07 \\
6.24 E-07 \\
5.85 E-07 \\
5.10 E-07 \\
5.12 E-07 \\
4.82 E-07 \\
2.01 E-07 \\
1.95 E-07 \\
2.36 E-07 \\
4.35 E-07\end{array}$ & $\begin{array}{l}B \\
D \\
C \\
C \\
C \\
D \\
D \\
D \\
D \\
F \\
F \\
F \\
F \\
F \\
G \\
G \\
D \\
F\end{array}$ & $\begin{array}{l}0.89 \\
4.70 \\
7.15 \\
0.89 \\
2.65 \\
0.89 \\
0.89 \\
0.89 \\
0.89 \\
0.89 \\
0.89 \\
0.89 \\
0.89 \\
0.89 \\
0.89 \\
0.89 \\
0.89 \\
0.89\end{array}$ \\
\hline
\end{tabular}


WHC-SD-CP-TI-197 Revision 0

$\begin{array}{llllllll}\text { ESE } & 30000 & 0 & 19.39 & 1 & 3.42 E-07 & 3.42 E-07 \mathrm{~F} & 0.89 \\ \text { SE } & 25200 & 0 & 11.01 & 1 & 3.60 E-07 & 3.60 E-07 \mathrm{~F} & 0.89 \\ \text { SSE } & 22900 & 0 & 4.75 & 1 & 3.40 E-07 & 3.40 E-07 \mathrm{D} & 0.89\end{array}$


WHC-SD-CP-TI-197 Revision 0

Gxa Version 4.0

December 19, 1994

General Purpose Atmospheric Dispersion Code

Produced by Westinghouse Hanford Company

Users Guide documented in UHC-SO-GN-SWD-30002 Rev. 1.

Validation documented in MHC-SD-EN-SWD-30003 ReV. 1.

Code Custodian is: Brit E. Hey

Uestinghouse Hanford Conpany

P.0. Box 1970

Richtand, UA 99352

(509) 376-2921

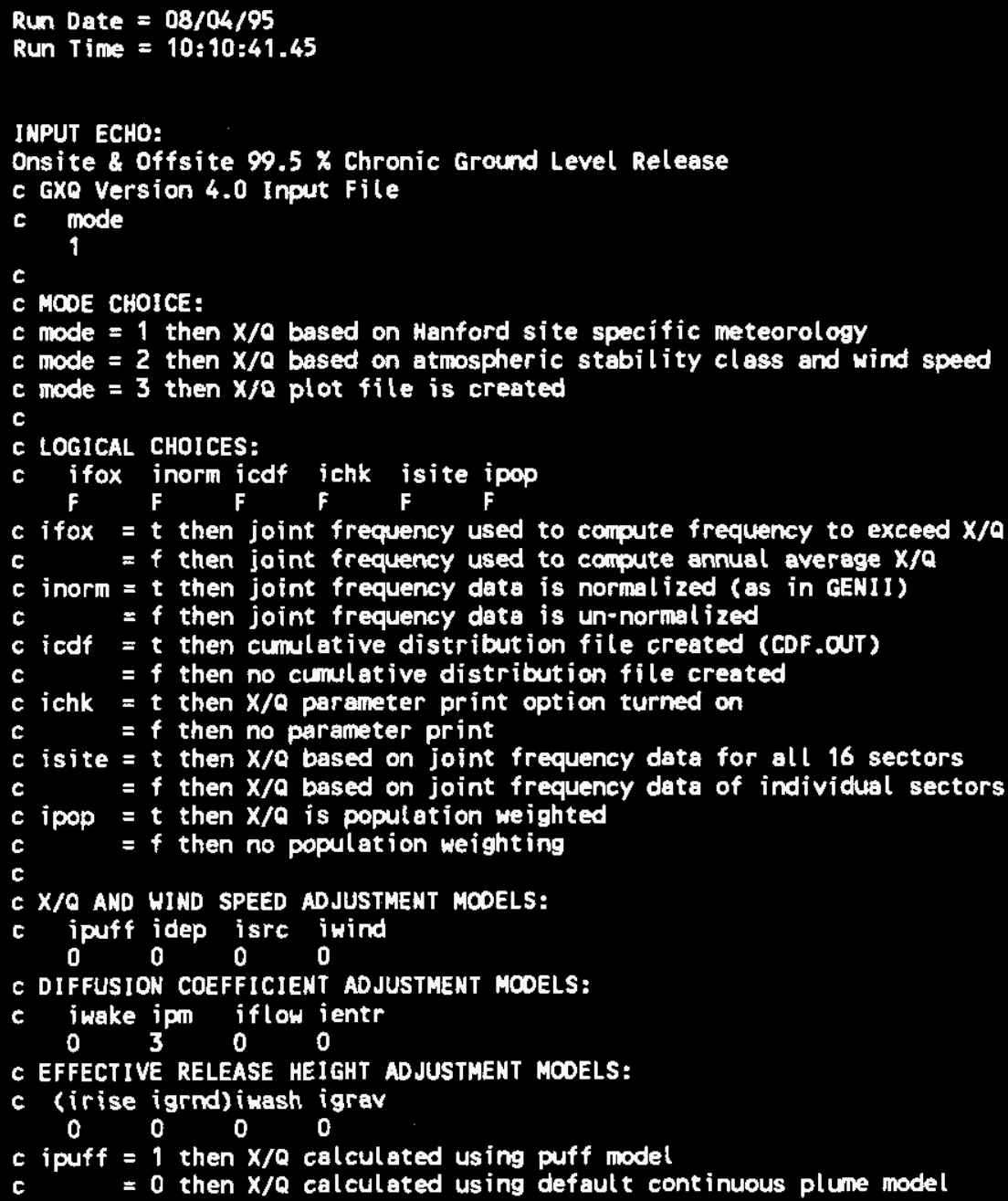




\section{WHC-SD-CP-TI-197 Revision 0}

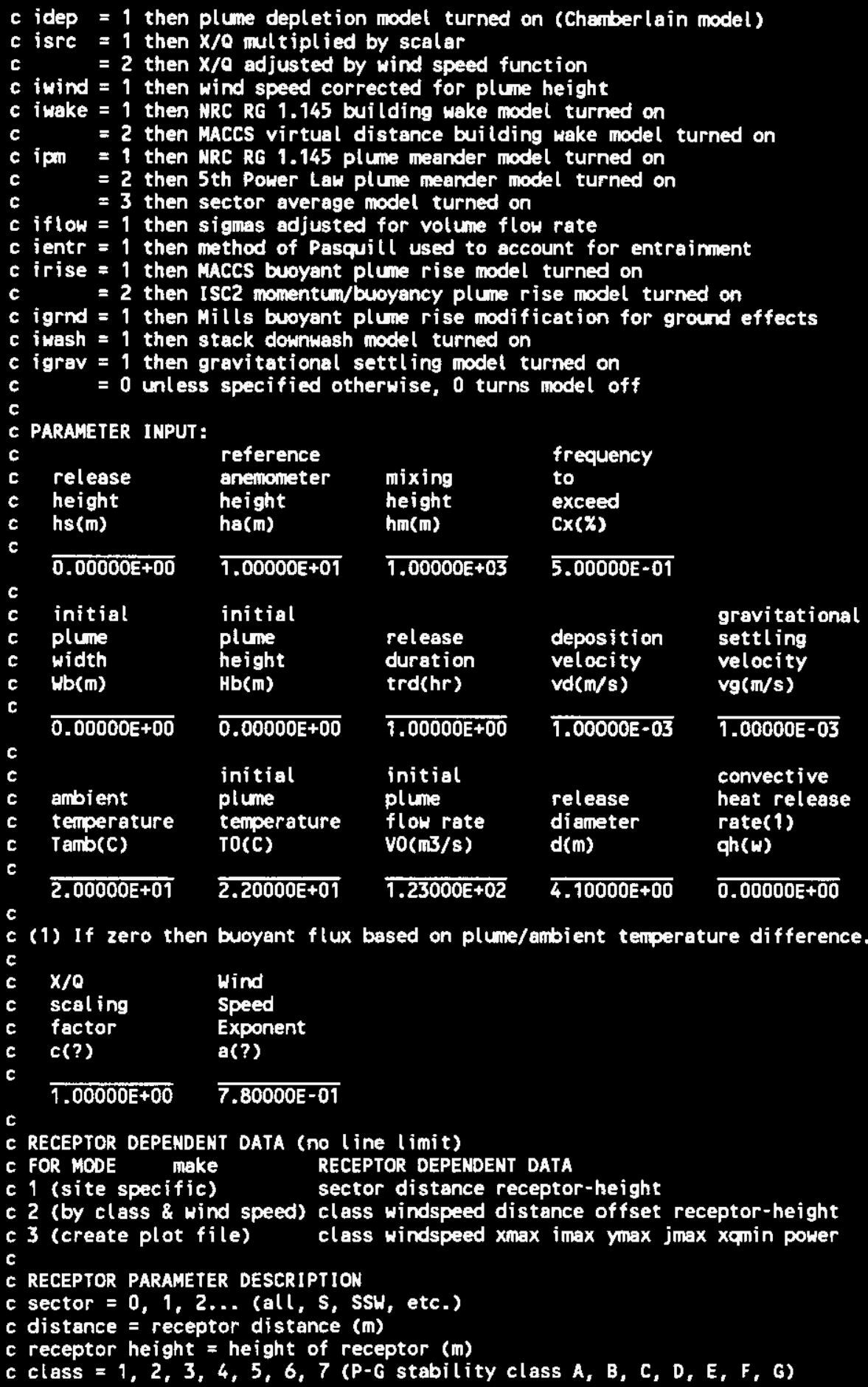




\section{WHC-SD-CP-TI-197 Revision 0}

c windspeed = anemoneter wind speed $(\mathrm{m} / \mathrm{s})$

c offset $=$ offset from plune centerline (m)

c $x \max =$ maximum distance to plot or calculate to (m)

c ingax $=$ distence intervals

c ymax $=$ maximum offset to plot (m)

c jmax = offset intervals

c $x q m i n=$ minimum scaled $X / Q$ to calculate

c power $=$ exponent in power function step size

MODE:

Site specific $X / O$ calculated.

LOGICAL CHOICES:

Joint frequency used to calculate annual average $\mathrm{X} / \mathrm{a}$.

No normalization of joint frequency.

$X / a$ calculated for single sector.

MODELS SELECTED:

Sector average model selected.

Default Gaussian plune model selected.

WARNIMG/ERROR MESSAGES:

JOINT FREQUENCY DATA:

200 AREA (HMS) - $10 \mathrm{H}$ - Pasquill A - G (1983 - 1991 Average)

Created 8/26/92 KR

Onsite \& Offsite $99.5 \times$ Chronic Ground Level Release

\begin{tabular}{|c|c|c|c|c|c|c|c|c|}
\hline SECTOR & $\begin{array}{l}\text { DISTANCE } \\
\text { (m) }\end{array}$ & $\begin{array}{l}\text { RECEPT } \\
\text { HEIGHT } \\
\text { (m) }\end{array}$ & $\begin{array}{l}\text { SECT. } \\
\text { FREO } \\
(X)\end{array}$ & POPULATION & $\begin{array}{c}\text { TOTAL } \\
\text { POPULATION } \\
\text { SCALED } \\
X / a \\
(s / m 3)\end{array}$ & $\begin{array}{l}\text { AVERAGE } \\
\text { INDIVIDUAL } \\
\text { SCALED } \\
x / a \\
(s / m 3)\end{array}$ & $\begin{array}{l}\text { ATM. } \\
\text { STAB. } \\
\text { CLASS }\end{array}$ & $\begin{array}{l}\text { UIND } \\
\text { SPEED } \\
\text { (m/s) }\end{array}$ \\
\hline $\begin{array}{l}\text { UNH } \\
\text { NE } \\
\text { ENE } \\
\text { ESE } \\
\text { SSE } \\
\text { S } \\
\text { SSH } \\
\text { SW } \\
\text { USH } \\
\text { W } \\
\text { WNH } \\
\text { NW } \\
\text { WNW } \\
\text { N } \\
\text { NWE } \\
\text { NE } \\
\text { ENE } \\
\text { E } \\
\text { ESE } \\
\text { SE } \\
\text { SSE }\end{array}$ & $\begin{array}{r}550 \\
1540 \\
750 \\
1050 \\
930 \\
15000 \\
15400 \\
16100 \\
13200 \\
12500 \\
13200 \\
16500 \\
17400 \\
19200 \\
26000 \\
28900 \\
25600 \\
25200 \\
30000 \\
25200 \\
22900\end{array}$ & $\begin{array}{l}0 \\
0 \\
0 \\
0 \\
0 \\
0 \\
0 \\
0 \\
0 \\
0 \\
0 \\
0 \\
0 \\
0 \\
0 \\
0 \\
0 \\
0 \\
0 \\
0 \\
0\end{array}$ & $\begin{array}{r}3.98 \\
3.90 \\
6.17 \\
18.80 \\
4.78 \\
6.30 \\
4.53 \\
2.93 \\
2.72 \\
4.80 \\
3.98 \\
4.72 \\
4.58 \\
4.36 \\
2.49 \\
3.90 \\
6.17 \\
14.05 \\
18.80 \\
10.83 \\
4.78\end{array}$ & $\begin{array}{l}\text { 1 } \\
1 \\
1 \\
1 \\
1 \\
1 \\
1 \\
1 \\
1 \\
1 \\
1 \\
1 \\
1 \\
1 \\
1 \\
1 \\
1 \\
1 \\
1 \\
1 \\
1 \\
1 \\
1\end{array}$ & $\begin{array}{l}6.00 E-06 \\
7.92 E-07 \\
3.65 E-06 \\
5.81 E-06 \\
2.47 E-06 \\
3.62 E-08 \\
2.09 E-08 \\
1.94 E-08 \\
2.57 E-08 \\
5.02 E-08 \\
3.85 E-08 \\
3.29 E-08 \\
3.12 E-08 \\
2.91 E-08 \\
1.07 E-08 \\
1.09 E-08 \\
1.72 E-08 \\
4.82 E-08 \\
3.95 E-08 \\
2.95 E-08 \\
1.85 E-08\end{array}$ & $\begin{array}{l}6.00 E-06 \\
7.92 E-07 \\
3.65 E-06 \\
5.81 E-06 \\
2.47 E-06 \\
3.62 E-08 \\
2.09 E-08 \\
1.94 E-08 \\
2.57 E-08 \\
5.02 E-08 \\
3.85 E-08 \\
3.29 E-08 \\
3.12 E-08 \\
2.91 E-08 \\
1.07 E-08 \\
1.09 E-08 \\
1.72 E-08 \\
4.82 E-08 \\
3.95 E-08 \\
2.95 E-08 \\
1.85 E-08\end{array}$ & & \\
\hline
\end{tabular}




\section{WHC-SD-CP-TI-197 Revision 0}

GXo Version 4.0

Decenber 19, 1994

General Purpose Atmospheric Dispersion Code

Produced by Hestinghouse Hanford Company

Users Guide documented in HHC-SD-GN-SWD-30002 ReV. 1.

Validation documented in UHC-SD-GN-SWD-30003 ReV. 1.

Code Custodian is: Brit E. Hey

West inghouse Hanford Company

P.0. Box 1970

Richland, UA 99352

(509) 376-2921

Run Date $=08 / 04 / 95$

Run Time $=08: 43: 22.77$

INPUT ECHO:

Onsite \& Offsite $99.5 \times$ Chronic stack Level Release c GXQ Version 4.0 Input file

c mode

$$
1
$$

c MODE CHOICE:

$c$ mode $=1$ then $X / Q$ based on Hanford site specific meteorology

$c$ mode $=2$ then $X / Q$ based on atmospheric stability class and wind speed

$c$ mode $=3$ then $X / Q$ plot file is created

c

c LOGICAL CHOICES:

c ifox inorm icdf ichk isite ipop

$F \quad F \quad F \quad F \quad F \quad F$

$c$ ifox $=t$ then joint frequency used to compute frequency to exceed $X / Q$

$c \quad=f$ then joint frequency used to compute annual average $x / a$

$c$ inorm $=t$ then joint frequency data is normalized (as in GENI $I$ )

$c \quad=f$ then joint frequency data is un-normalized

$c$ icdf $=t$ then cumulative distribution file created (CDF.OUT)

$c \quad=f$ then no cumulative distribution file created

$c$ ichk $=t$ then $X / 0$ parameter print option turned on

$=f$ then no parameter print

isite $=t$ then $x / a$ based on joint frequency data for all 16 sectors

$=f$ then $x / 0$ based on joint frequency data of individual sectors

ipop $=t$ then $x / Q$ is population weighted

$=f$ then no population weighting

$X / O$ AND WIND SPEED ADJUSTMENT MODELS:

c ipuff idep isrc iwind

$\begin{array}{llll}0 & 0 & 0 & 1\end{array}$

c DIFFUSION COEFFICIENT ADJUSTMENT MODELS:

C iwake ipm iflow ientr

$\begin{array}{llll}0 & 3 & 1 & 0\end{array}$

c EFFECTIVE RELEASE HEIGHT ADJUSTMENT MOOELS:

c (irise igrnd)iwash igrav
2 10

$c$ ipuff $=1$ then $X / 0$ calculated using puff model

$c \quad=0$ then $X / Q$ calculated using default continuous plume model 


\section{WHC-SD-CP-TI-197 Revision 0}

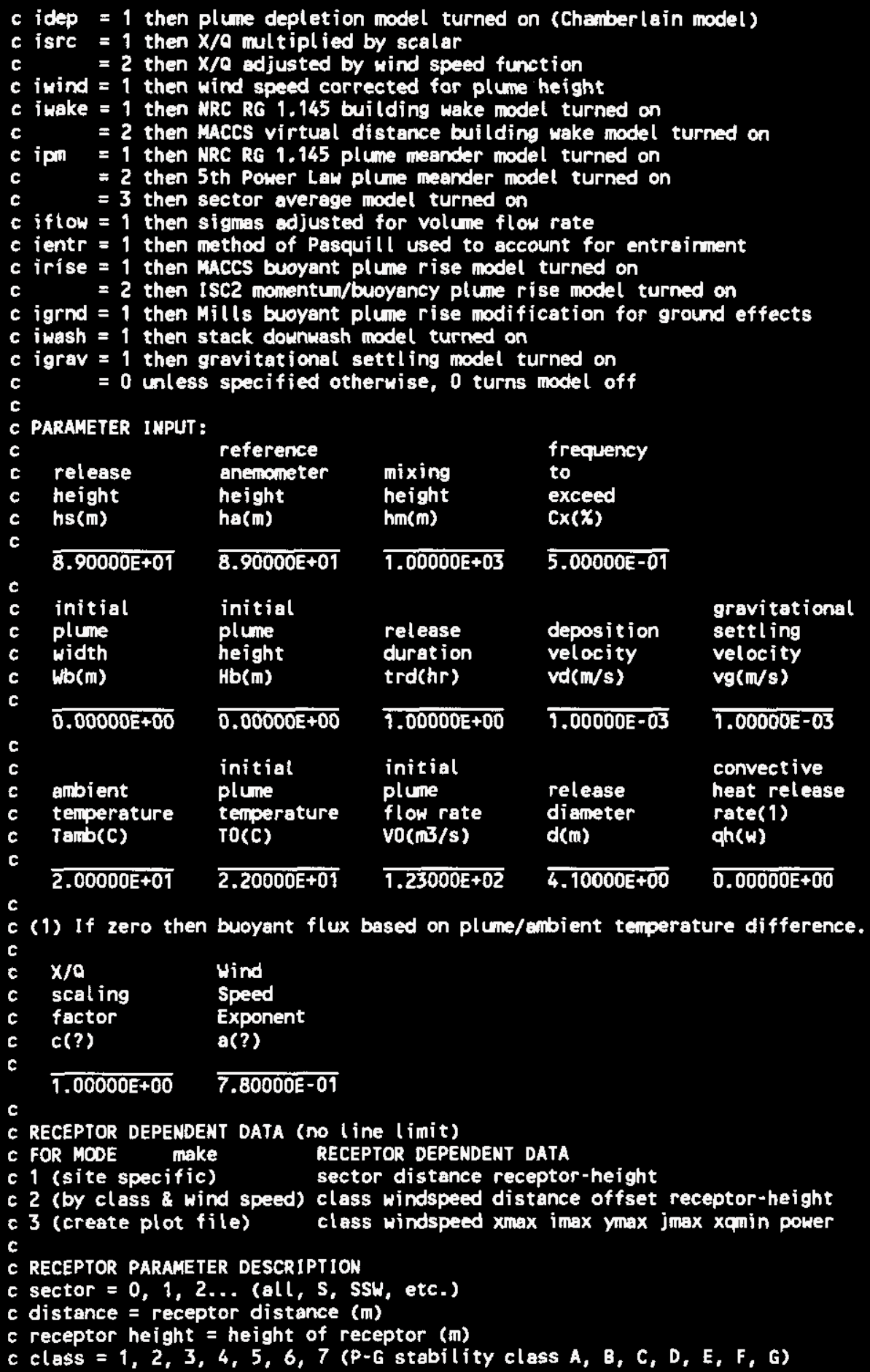




\section{WHC-SD-CP-TI-197 Revision 0}

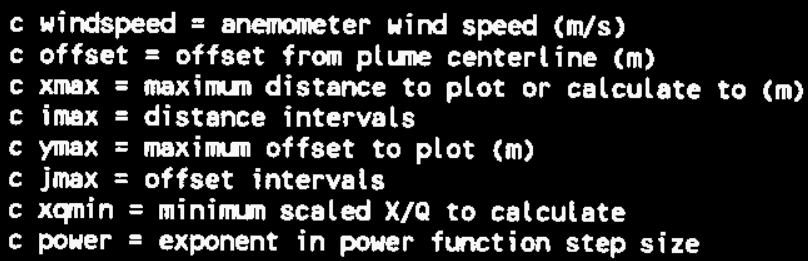

MODE:

Site specific $X / Q$ calculated.

LOGICAL CHOICES:

Joint frequency used to calculate annual average $x / 0$.

No normal ization of joint frequency.

$x / 0$ calculated for single sector.

MODELS SELECTED:

Flow adjustment model.

Sector average model selected.

ISC2 momentum/buoyancy plune model based on temperature difference.

Stack downuash model selected.

Default Gaussian plume model selected.

Wind velocity corrected for average plune height.

UARHING/ERROR MESSAGES:

WARNING $\# 10$ - $x / 0$ may be underestimated for plune rise scenario without accounting for entrainment.

JOINT FREQUENCY DATA:

200 AREA (HNS) - 89 M - Pasquill A - G (1983 - 1991 Average)

Created $8 / 26 / 92 \mathrm{KR}$

Onsite \& Offsite $99.5 \times$ Chronic stack Level Release

\begin{tabular}{|c|c|c|c|c|c|c|c|c|}
\hline SECTOR & $\begin{array}{l}\text { DISTANCE } \\
\text { (m) }\end{array}$ & $\begin{array}{l}\text { RECEPT } \\
\text { HEIGHT } \\
\text { (m) }\end{array}$ & $\begin{array}{l}\text { SECT. } \\
\text { FREQ. } \\
\text { (X) }\end{array}$ & POPULATION & $\begin{array}{c}\text { TOTAL } \\
\text { POPULATION } \\
\text { SCALED } \\
x / 9 \\
\left(s / m^{3}\right)\end{array}$ & $\begin{array}{l}\text { AVERAGE } \\
\text { INDIVIDUAL } \\
\text { SCALED } \\
x / 0 \\
(\mathrm{~s} / \mathrm{m} 3)\end{array}$ & $\begin{array}{l}\text { ATM. } \\
\text { STAB. } \\
\text { CLASS }\end{array}$ & $\begin{array}{l}\text { WIND } \\
\text { SPEED } \\
\text { (m/s) }\end{array}$ \\
\hline $\begin{array}{l}\text { WNW } \\
\text { NE } \\
\text { ENE } \\
\text { ESE } \\
\text { SSE } \\
\text { S } \\
\text { SSW } \\
\text { SH } \\
\text { WSW } \\
\text { W } \\
\text { WNW } \\
\text { NW } \\
\text { NNU } \\
\text { N } \\
\text { NNE } \\
\text { NE } \\
\text { ENE }\end{array}$ & \begin{tabular}{r|}
630 \\
1540 \\
830 \\
1060 \\
930 \\
15000 \\
15400 \\
16100 \\
13200 \\
12500 \\
13200 \\
16500 \\
17400 \\
19200 \\
26000 \\
28900 \\
25600 \\
\end{tabular} & $\begin{array}{l}0 \\
0 \\
0 \\
0 \\
0 \\
0 \\
0 \\
0 \\
0 \\
0 \\
0 \\
0 \\
0 \\
0 \\
0 \\
0 \\
0\end{array}$ & $\begin{array}{r}3.81 \\
3.89 \\
6.27 \\
19.41 \\
4.73 \\
6.12 \\
4.29 \\
2.82 \\
2.57 \\
4.58 \\
3.81 \\
4.58 \\
4.52 \\
4.33 \\
2.48 \\
3.89 \\
6.27\end{array}$ & $\begin{array}{l}1 \\
1 \\
1 \\
1 \\
1 \\
1 \\
1 \\
1 \\
1 \\
1 \\
1 \\
1 \\
1 \\
1 \\
1 \\
1 \\
1\end{array}$ & $\begin{array}{l}5.63 E-08 \\
8.90 E-09 \\
2.42 E-08 \\
2.77 E-08 \\
3.28 E-08 \\
9.69 E-09 \\
6.05 E-09 \\
4.99 E-09 \\
5.86 E-09 \\
1.03 E-08 \\
7.83 E-09 \\
7.04 E-09 \\
6.23 E-09 \\
5.47 E-09 \\
2.37 E-09 \\
2.65 E-09 \\
3.92 E-09\end{array}$ & $\begin{array}{l}5.63 E-08 \\
8.90 E-09 \\
2.42 E-08 \\
2.77 E-08 \\
3.28 E-08 \\
9.69 E-09 \\
6.05 E-09 \\
4.99 E-09 \\
5.86 E-09 \\
1.03 E-08 \\
7.83 E-09 \\
7.04 E-09 \\
6.23 E-09 \\
5.47 E-09 \\
2.37 E-09 \\
2.65 E-09 \\
3.92 E-09\end{array}$ & & \\
\hline
\end{tabular}




\section{WHC-SD-CP-TI-197 Revision 0}

\begin{tabular}{|c|c|c|c|c|c|c|}
\hline $\begin{array}{l}\text { E } \\
\text { ESE } \\
\text { SE } \\
\text { SSE }\end{array}$ & $\begin{array}{l}25200 \\
30000 \\
25200 \\
22900\end{array}$ & $\begin{array}{l}0 \\
0 \\
0 \\
0\end{array}$ & $\begin{array}{r}14.38 \\
19.41 \\
11.04 \\
4.73\end{array}$ & $\begin{array}{l}1 \\
1 \\
1 \\
1\end{array}$ & $\begin{array}{l}9.21 E-09 \\
9.47 E-09 \\
7.86 E-09 \\
5.29 E-09\end{array}$ & $\begin{array}{l}9.21 \mathrm{E}-09 \\
9.47 \mathrm{E}-09 \\
7.86 \mathrm{E}-09 \\
5.29 \mathrm{E}-09\end{array}$ \\
\hline
\end{tabular}


GXa Version 4.0

December 19, 1994

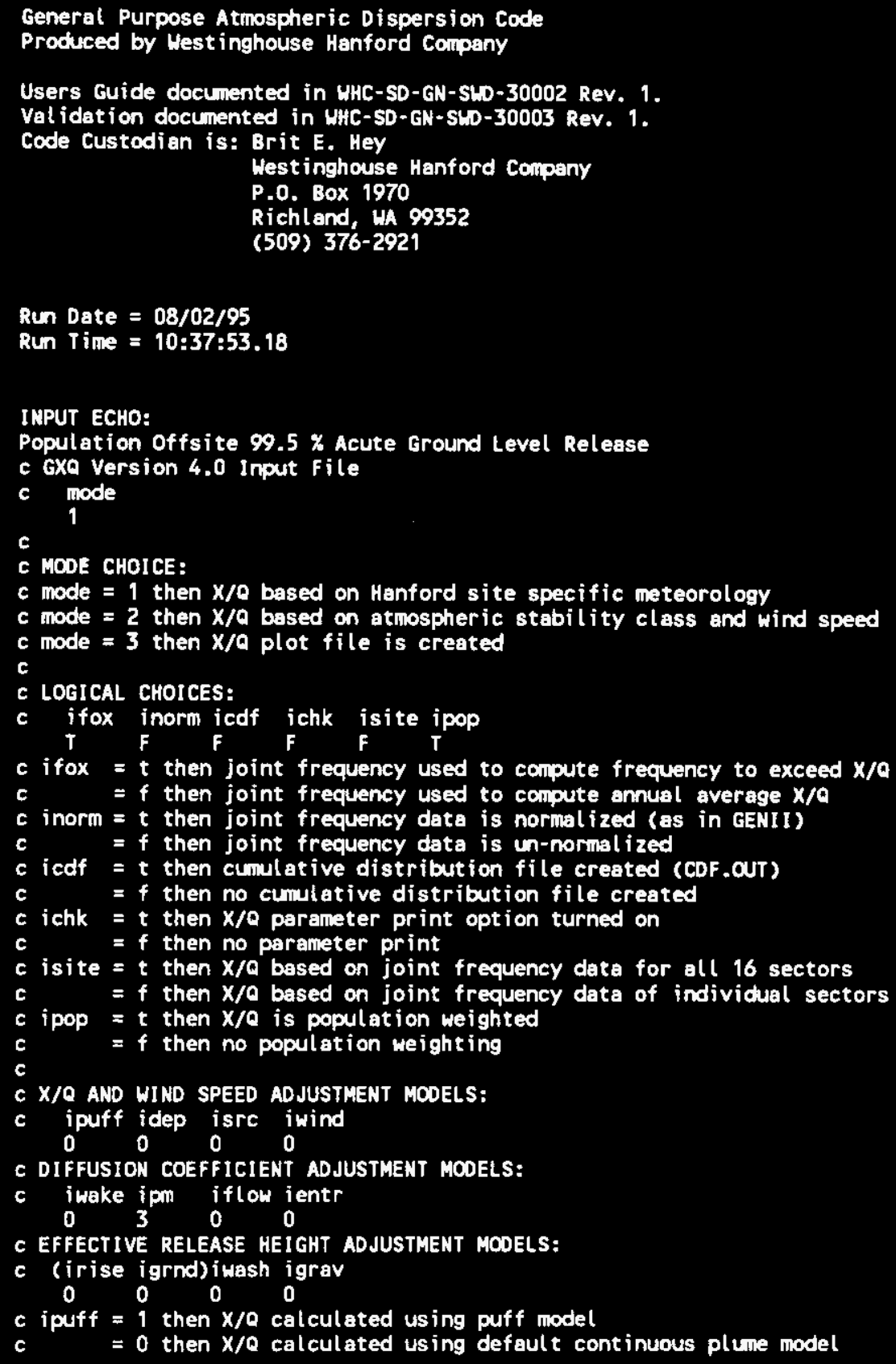




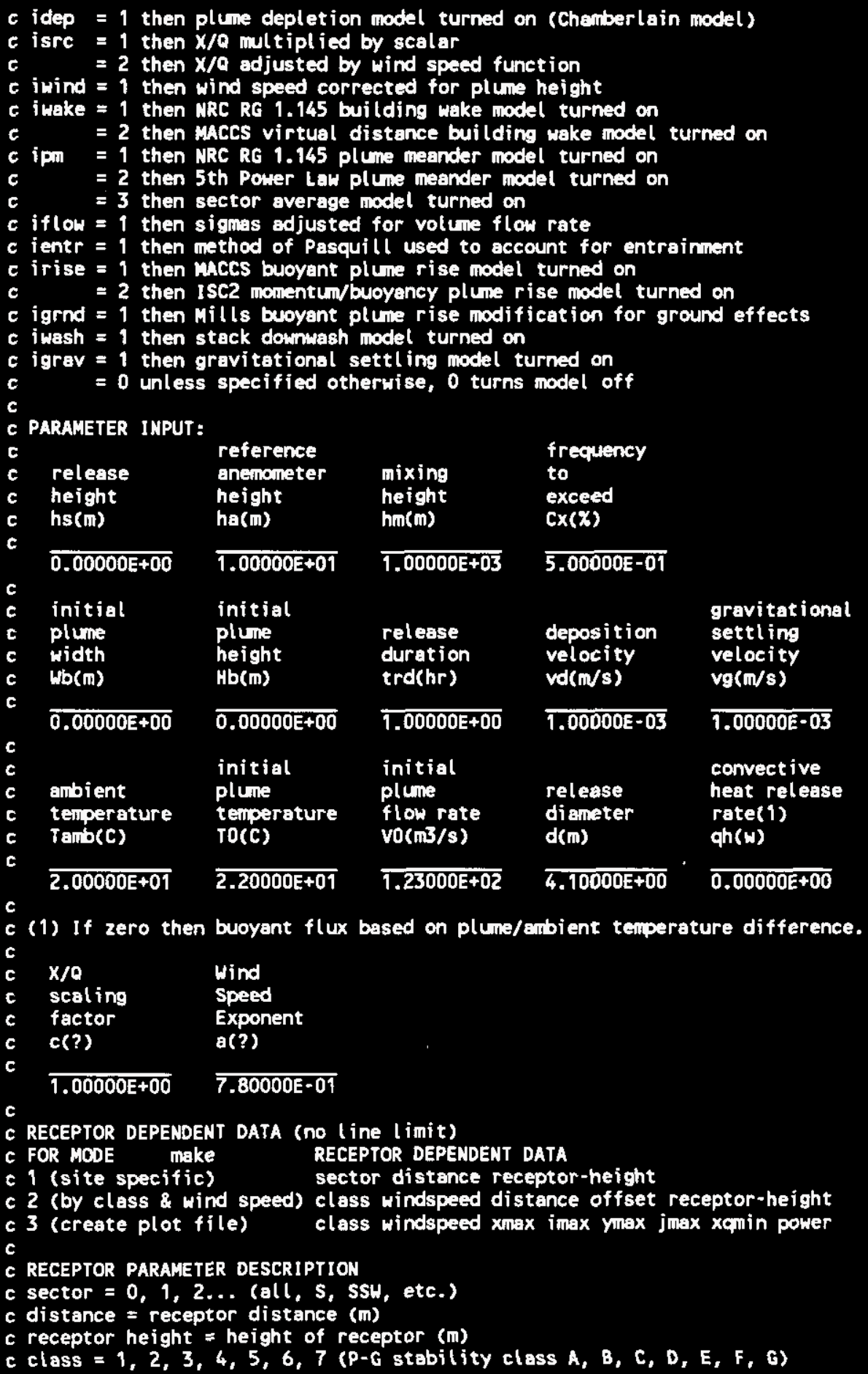




\section{WHC-SD-CP-TI-197 Revision 0}

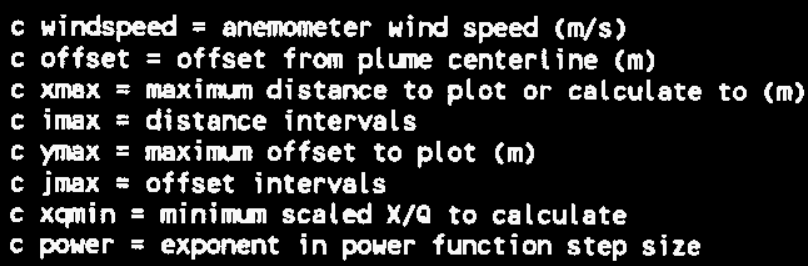

MOOE:

Site specific $\times / a$ calculated.

LOGICAL CHOICES:

Joint frequency used to calculate $x / Q$ based on frequency of exceedance.

No normal ization of joint frequency.

$x / a$ calculated for single sector.

$X / Q$ is population weighted.

MODELS SELECTED:

Sector average model selected.

Defaut t Gaussian plune model selected.

WARNING/ERROR MESSAGES:

JOINT FREQUENCY DATA:

200 AREA (HMS) - $10 \mathrm{M}$ - Pasquill A - G (1983 - 1991 Average)

Created 8/26/92 KR

POPULATION DATA:

Population within $80 \mathrm{~km}$ of the Hanford 200 Area, 1990 Census (Beck, et al 1991) Created 10/21/91 KR, Revised 2/4/92 KR

Population Offsite $99.5 \times$ Acute Ground Level Release

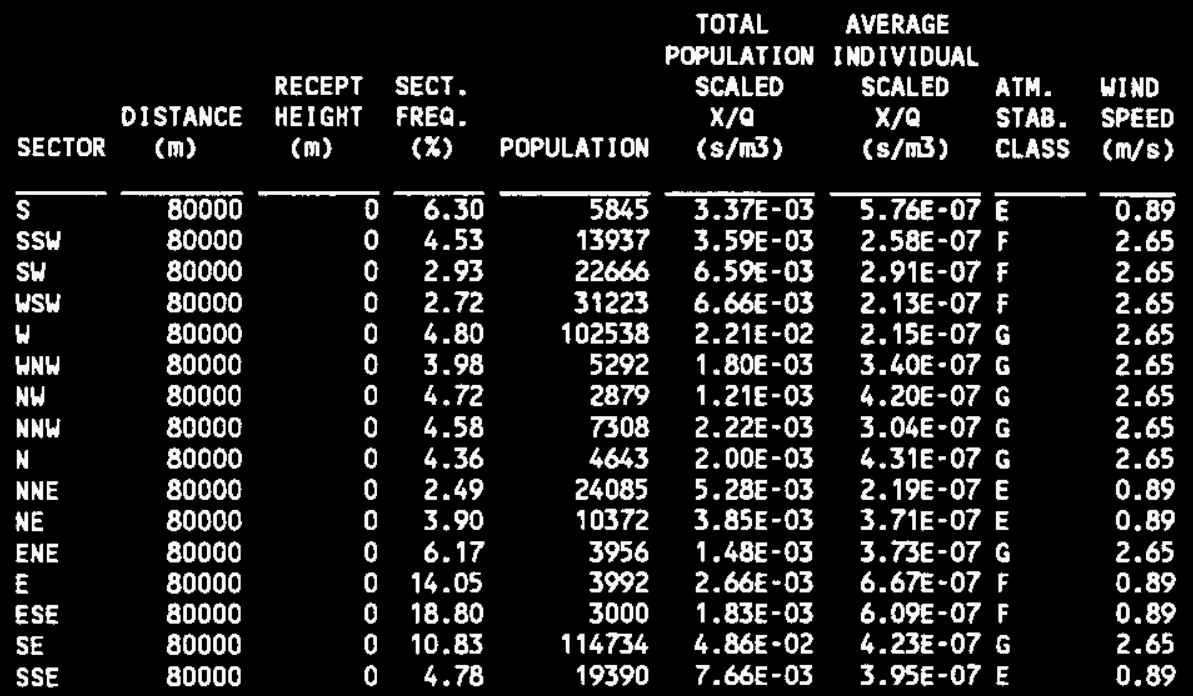




\section{WHC-SD-CP-TI-197 Revision 0}

GXo Version 4.0

December 19, 1994

General Purpose Atmospheric Dispersion Code Produced by West inghouse Hanford Company

Users Guide docunented in UHC-SD-GN-SW-30002 Rev. 1. Validation documented in UHC-SD-EN-SWD-30003 ReV. 1.

Code Custodian is: Brit E. Hey

West inghouse Hanford Company

P.0. Box 1970

Richland, WA 99352

(509) 376-2921

Run Date $=08 / 04 / 95$

Run $\operatorname{Tine}=08: 29: 53.60$

INPUT ECHO:

Population Offsite $99.5 \times$ Acute Stack Level Release

c QXa Version 4.0 Input file

c mode

1

c

c MODE CHOICE:

$c$ mode $=1$ then $X / Q$ based on Hanford site specific meteorology

$c$ mode $=2$ then $X / 0$ based on atmospheric stability class and uind speed

$c$ mode $=3$ then $x / 0$ plot file is created

c

c LOGICAL CHOICES:

c ifox inorm icdf ichk isite ipop

$c$ ifox $=t$ then joint frequency used to compute frequency to exceed $X / a$

$c \quad=f$ then joint frequency used to compute anmul average $x / a$

$c$ inorm $=t$ then joint frequency data is normalized (as in GENII)

$c \quad=f$ then joint frequency data is un-normalized

$c$ icdf $=t$ then cumulative distribution file created (CDF.OUT)

$c \quad=f$ then no cumulative distribution file created

c ichk $=t$ then $x / Q$ parameter $p r i n t$ option turned on

$=f$ then no paraneter print

$c$ isite $=t$ then $X / 0$ based on joint frequency data for all 16 sectors

$c \quad=f$ then $x / a$ based on joint frequency data of individual sectors

$c$ ipop $=t$ then $X / Q$ is population weighted

$c=f$ then no population weight ing

C X/O AND WIND SPEED ADJUSTMENT MOOELS:

c ipuff idep isrc iwind

$0 \quad 0 \quad 0 \quad 1$

c DIFFUSION COEFFICIENT ADJUSTMENT MOOELS:

C iwake ipm iflow ientr

$\begin{array}{llll}0 & 3 & 1 & 0\end{array}$

C EFFECTIVE RELEASE HEIGHT ADJUSTMENT MOOELS:

c (irise igrnd)iwash igrav

20010

$c$ ipuff $=1$ then $x / Q$ calculated using puff model

c $\quad=0$ then $X / O$ calculated using defaul $t$ continuous plume model 


\section{WHC-SD-CP-TI-197 Revision 0}

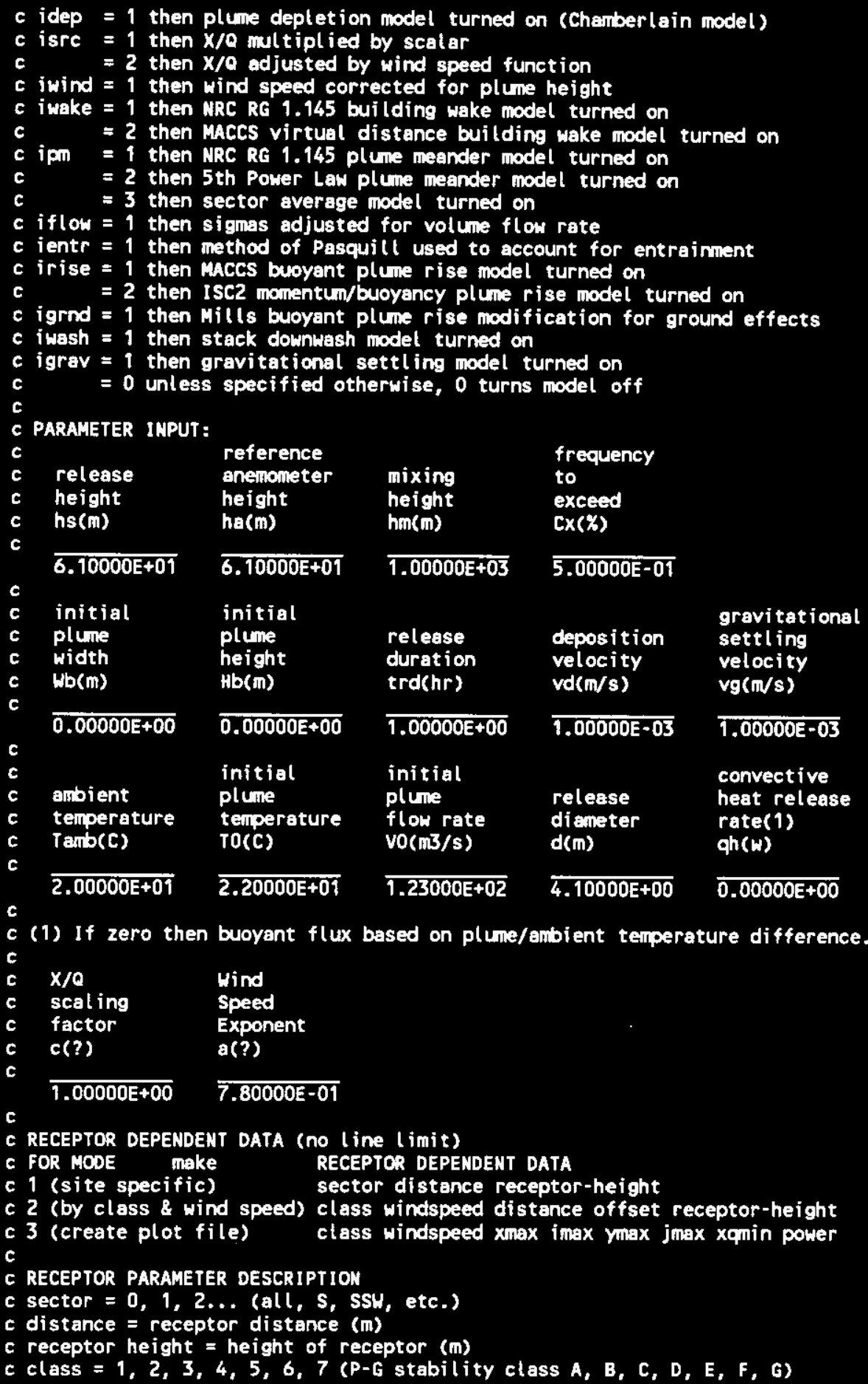




\section{WHC-SD-CP-TI-197 Revision 0}

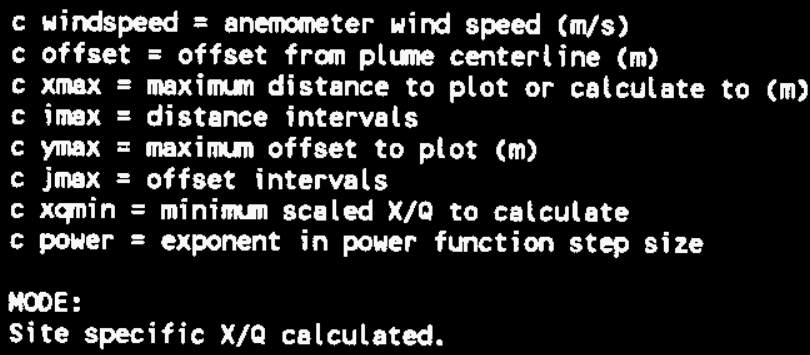

MOOELS SELECTED:

Flow adjustment model.

Sector average model selected.

ISC2 momentunbuoyancy plume model based on temperature difference.

Stack downwash model selected.

Default Gaussian plume model selected.

Wind velocity corrected for average plume height.

UARNIMG/ERROR MESSAGES:

UARNING \#10 - X/a may be underestimated for plume rise scenario without account ing for entrainment.

JOINT FREQUENCY DATA:

200 AREA (HMS) - 61 M - Pasquill A - G (1983 - 1991 Average)

Created $8 / 26 / 92$ KR

POPULATION DATA:

Population within $80 \mathrm{~km}$ of the Henford 200 Area, 1990 Census (Beck, et at 1991) Created 10/21/91 KR, Revised 2/4/92 KR

Population Offsite $99.5 \times$ Acute Stack Level Release

\begin{tabular}{|c|c|c|c|c|c|c|c|c|}
\hline ECTOR & $\begin{array}{l}\text { DISTANCE } \\
\text { (m) }\end{array}$ & $\begin{array}{l}\text { RECEPT } \\
\text { HEIGHT } \\
\text { (m) }\end{array}$ & $\begin{array}{l}\text { SECT. } \\
\text { FREQ. } \\
(X)\end{array}$ & POPULATIOW & $\begin{array}{l}\text { TOTAL } \\
\text { POPULATION } \\
\text { SCALED } \\
\text { X/a } \\
(\mathrm{s} / \mathrm{m} 3)\end{array}$ & $\begin{array}{c}\text { AVERAEE } \\
\text { INDIVIDUAL } \\
\text { SCALED } \\
\text { X/0 } \\
(s / m 3)\end{array}$ & $\begin{array}{l}\text { ATM. } \\
\text { STAB. } \\
\text { CLASS }\end{array}$ & $\begin{array}{l}\text { HINO } \\
\text { SPEED } \\
(m / s)\end{array}$ \\
\hline $\begin{array}{l}\text { S } \\
\text { SSH } \\
\text { SH } \\
\text { WSH } \\
\text { W } \\
\text { WWH } \\
\text { NW } \\
\text { WNH } \\
\text { N } \\
\text { WNE } \\
\text { NE } \\
\text { ENE }\end{array}$ & $\begin{array}{l}80000 \\
80000 \\
80000 \\
80000 \\
80000 \\
80000 \\
80000 \\
80000 \\
80000 \\
80000 \\
80000 \\
80000\end{array}$ & $\begin{array}{l}0 \\
0 \\
0\end{array}$ & $\begin{array}{l}6.11 \\
4.33 \\
2.79 \\
2.54 \\
4.56 \\
3.80 \\
4.58 \\
4.51 \\
4.31 \\
2.48 \\
3.95 \\
6.33\end{array}$ & $\begin{array}{r}5845 \\
13937 \\
22666 \\
31223 \\
102538 \\
5292 \\
2879 \\
7308 \\
4643 \\
24085 \\
10372 \\
3956\end{array}$ & $\begin{array}{l}1.54 E-03 \\
2.05 E-03 \\
3.42 E-03 \\
3.47 E-03 \\
1.37 E-02 \\
8.06 E-04 \\
5.73 E-04 \\
1.28 E-03 \\
9.05 E-04 \\
1.88 E-03 \\
1.35 E-03 \\
5.58 E-04\end{array}$ & $\begin{array}{l}2.64 E-07 \\
1.47 E-07 \\
1.51 E-07 \\
1.11 E-07 \\
1.33 E-07 \\
1.52 E-07 \\
1.99 E-07 \\
1.75 E-07 \\
1.95 E-07 \\
7.82 E-08 \\
1.30 E-07 \\
1.41 E-07\end{array}$ & $\begin{array}{l}D \\
D \\
D \\
D \\
G \\
G \\
G \\
E \\
E \\
D \\
D \\
G\end{array}$ & $\begin{array}{l}0.89 \\
0.89 \\
0.89 \\
0.89 \\
0.89 \\
0.89 \\
0.89 \\
0.89 \\
0.89 \\
0.89 \\
0.89 \\
0.89\end{array}$ \\
\hline
\end{tabular}


WHC-SD-CP-TI-197 Revision 0

$\begin{array}{llrrrrrr}\text { E } & 80000 & 0 & 14.39 & 3992 & 9.79 E-04 & 2.45 E-07 \mathrm{E} & 0.89 \\ \text { ESE } & 80000 & 0 & 19.39 & 3000 & 6.60 E-04 & 2.20 E-07 \mathrm{E} & 0.89 \\ \text { SE } & 80000 & 0 & 11.01 & 114734 & 1.85 E-02 & 1.61 E-07 \mathrm{G} & 0.89 \\ \text { SSE } & 80000 & 0 & 4.75 & 19390 & 2.85 E-03 & 1.47 E-07 \mathrm{G} & 0.89\end{array}$


EXa Version 4.0

Decenber 19, 1994

General Purpose Atmospheric Dispersion Code

Produced by Westinghouse Hanford Company

Users Guide documented in UHC-SD-GN-SWD-30002 Rev. 1.

Validation documented in WHC-SD-GN-SWD-30003 ReV. 1.

Code Custodian is: Brit E. Hey

West inghouse Hanford Company

P.O. Box 1970

Richtand, WA 99352

(509) 376-2921

Run Date $=08 / 02 / 95$

Run Time $=10: 56: 38.99$

INPUT ECHO:

Population offsite $99.5 \times$ Chronic Ground Level Release

c EXa Version 4.0 Input File

c mode

1

c

c MODE CHOICE:

$c$ mode $=1$ then $X / a$ based on Hanford site specific meteorology

$c$ mode $=2$ then $X / a$ based on atmospheric stability class and wind speed

$c$ mode $=3$ then $X / Q$ plot file is created

c

c LOGICAL CHOICES:

c ifox inorm icdf ichk isite ipop

$\begin{array}{llllll}F & F & F & F & F & T\end{array}$

$c$ ifox $=t$ then joint frequency used to compute frequency to exceed $x / Q$

c $\quad f$ then joint frequency used to compute annual average $x / 0$

c inorm $=t$ then joint frequency date is normal ized (as in GENII)

$c \quad=f$ then joint frequency data is un-normal ized

$c$ icdf $=t$ then cumulative distribution file created (CDF.OUT)

$c=f$ then no cumulative distribution file created

c ichk $=t$ then $X / Q$ parameter print option turned on

c $\quad=f$ then no parameter print

$c$ isjte $=t$ then $X / 0$ based on joint frequency data for all 16 sectors

$c=f$ then $X / Q$ based on joint frequency data of individual sectors

$c$ ipop $=t$ then $X / Q$ is population weighted

$c \quad=f$ then no population weighting

C $X / Q$ AND WIND SPEED ADJUSTMENT MODELS:

c ipuff idep isrc iwind

$\begin{array}{llll}0 & 0 & 0 & 0\end{array}$

C DIFFUSION COEFFICIENT ADJUSTMENT MODELS:

c iwake ipn iflow ientr

$\begin{array}{llll}0 & 3 & 0 & 0\end{array}$

c EFFECTIVE RELEASE HEIGHT ADJUSTMENT MODELS:

c (irise igrnd) iwash igrav

$$
\begin{array}{llll}
0 & 0 & 0 & 0
\end{array}
$$

$c$ ipuff $=1$ then $x / \theta$ calculated using puff model

$c \quad=0$ then $X / Q$ calculated using default cont inuous plume model 


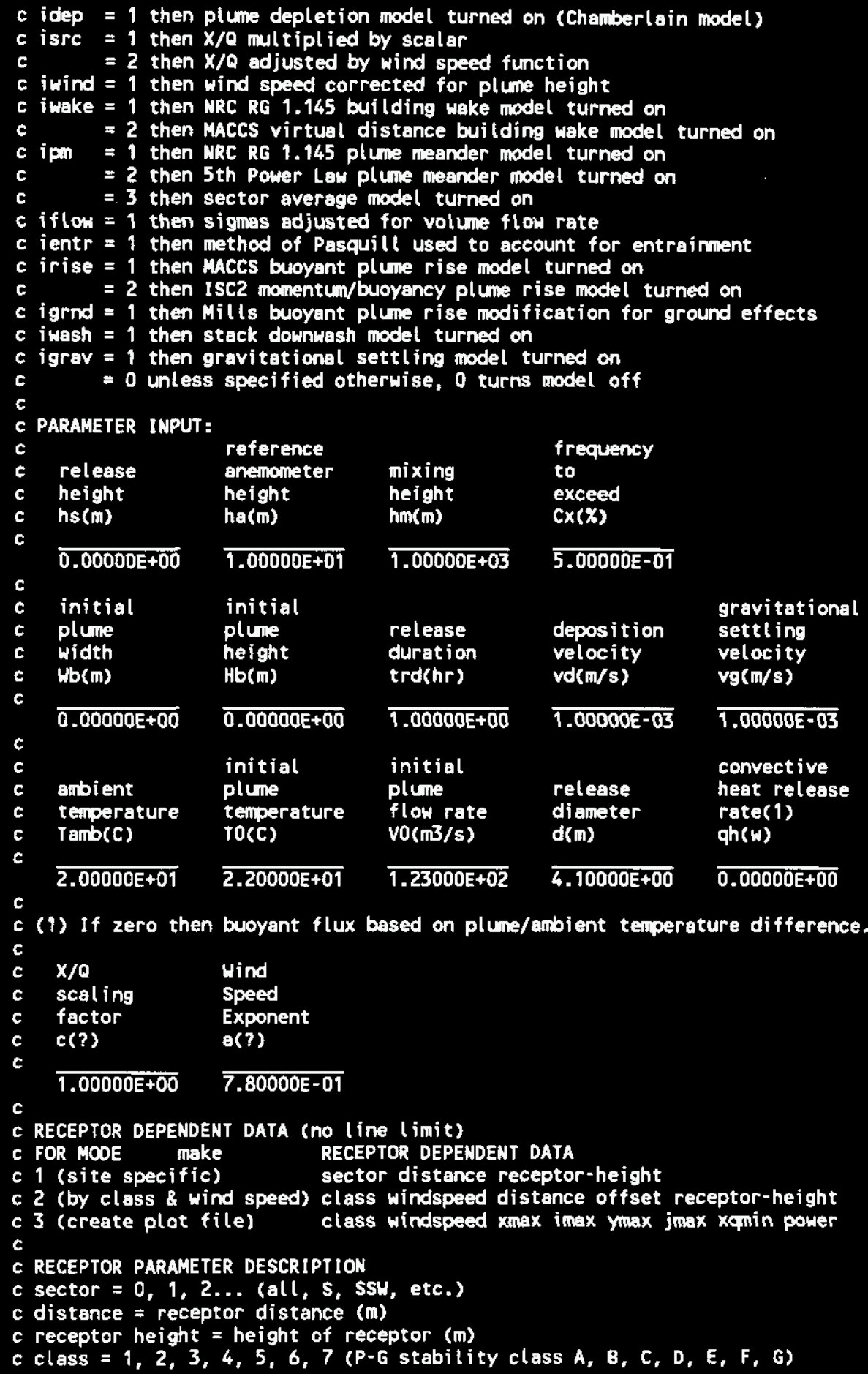




\title{
WHC-SD-CP-TI-197 Revision 0
}

\author{
c windspeed = anemoneter wind speed $(\mathrm{m} / \mathrm{s})$ \\ c offset $=$ offset from plume centerl ine (m) \\ c $x \max =$ maximun distance to plot or calculate to $(\mathrm{m})$ \\ c $i \max =$ distance intervals \\ c ymax = maximm offset to plot (m) \\ c $\mathrm{jmax}=$ offset intervals \\ c $x q \min =$ minimun scaled $x / 0$ to calculate \\ $c$ power $=$ exponent in power function step size \\ MODE: \\ Site specific $X / Q$ calculated. \\ LOGICAL CHOICES: \\ Joint frequency used to calculate annual average $x / 0$. \\ No normal ization of joint frequency. \\ $x / a$ calculated for single sector. \\ $X / Q$ is population weighted. \\ MODELS SELECTED: \\ Sector average model selected. \\ Default Gaussian plune nodel selected. \\ UARNING/ERROR MESSAGES:

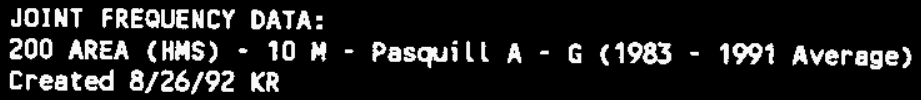

Population Offsite $99.5 \times$ Chronic Ground Level Release

\begin{tabular}{|c|c|c|c|c|c|c|c|c|}
\hline SECTOR & $\begin{array}{l}\text { DISTANCE } \\
\text { (m) }\end{array}$ & $\begin{array}{l}\text { RECEPT } \\
\text { HEIGHT } \\
\text { (m) }\end{array}$ & $\begin{array}{l}\text { SECT. } \\
\text { FREQ. } \\
(\%)\end{array}$ & POPULATION & $\begin{array}{c}\text { TOTAL } \\
\text { POPULATION } \\
\text { SCALED } \\
X / 0 \\
(s / m 3)\end{array}$ & $\begin{array}{l}\text { AVERAGE } \\
\text { INDIVIDUAL } \\
\text { SCALED } \\
X / 0 \\
\left(s / \mathrm{m}^{3}\right)\end{array}$ & $\begin{array}{l}\text { ATM. } \\
\text { STAB, } \\
\text { CLASS }\end{array}$ & $\begin{array}{l}\text { UIND } \\
\text { SPEED } \\
(\mathrm{m} / \mathrm{s})\end{array}$ \\
\hline $\begin{array}{l}\text { S } \\
\text { SSH } \\
\text { SW } \\
\text { WSH } \\
\text { W } \\
\text { WWH } \\
\text { NW } \\
\text { NWW } \\
\text { N } \\
\text { NWE } \\
\text { NE } \\
\text { ENE } \\
\text { E } \\
\text { ESE } \\
\text { SE } \\
\text { SSE }\end{array}$ & $\begin{array}{l}80000 \\
80000 \\
80000 \\
80000 \\
80000 \\
80000 \\
80000 \\
80000 \\
80000 \\
80000 \\
80000 \\
80000 \\
80000 \\
80000 \\
80000 \\
80000\end{array}$ & $\begin{array}{l}0 \\
0 \\
0 \\
0 \\
0 \\
0 \\
0 \\
0 \\
0 \\
0 \\
0 \\
0 \\
0 \\
0 \\
0 \\
0\end{array}$ & $\begin{array}{r}6.30 \\
4.53 \\
2.93 \\
2.72 \\
4.80 \\
3.98 \\
4.72 \\
4.58 \\
4.36 \\
2.49 \\
3.90 \\
6.17 \\
14.05 \\
18.80 \\
10.83 \\
4.78\end{array}$ & $\begin{array}{r}5845 \\
13937 \\
22666 \\
31223 \\
102538 \\
5292 \\
2879 \\
7308 \\
4643 \\
24085 \\
10372 \\
3956 \\
3992 \\
3000 \\
114734 \\
19390\end{array}$ & $\begin{array}{l}7.40 E-05 \\
7.77 E-05 \\
1.27 E-04 \\
1.27 E-04 \\
5.01 E-04 \\
3.52 E-05 \\
2.64 E-05 \\
4.86 E-05 \\
3.68 E-05 \\
7.73 E-05 \\
6.40 E-05 \\
3.20 E-05 \\
9.06 E-05 \\
7.38 E-05 \\
1.36 E-03 \\
1.55 E-04\end{array}$ & $\begin{array}{l}1.27 E-08 \\
5.57 E-09 \\
5.60 E-09 \\
4.08 E-09 \\
4.88 E-09 \\
6.65 E-09 \\
9.18 E-09 \\
6.65 E-09 \\
7.93 E-09 \\
3.21 E-09 \\
6.17 E-09 \\
8.08 E-09 \\
2.27 E-08 \\
2.46 E-08 \\
1.18 E-08 \\
8.00 E-09\end{array}$ & & \\
\hline
\end{tabular}


EXa Version 4.0

Decenber 19, 1994

General Purpose Atmospheric Dispersion Code

Produced by Westinghouse Hanford Company

Users Guide documented in WHC-SD-GN-SWD-30002 Rev. 1.

Val idation documented in WHC-SD-GN-SWD-30003 ReV. 1.

Code Custodian is: Brit E. Hey

West inghouse Hanford Company

P.O. Box 1970

Richland, UA 99352

(509) 376-2921

Run Date $=08 / 04 / 95$

Run $\operatorname{Time}=08: 31: 06.55$

INPUT ECHO:

Population Offsite $99.5 \times$ Chronic Stack Level Retease

c GXe Version 4.0 Input file

c mode

1

c MODE CHOICE:

$c$ mode $=1$ then $X / Q$ based on Hanford site specific meteorology

$c$ mode $=2$ then $X / Q$ based on atmospheric stability class and wind speed

$c$ mode $=3$ then $X / Q$ plot file is created

c

c LOGICAL CHOICES:

c ifox inorm icdf ichk isite ipop

$F \quad F \quad F \quad F \quad F \quad T$

$c$ ifox $=t$ then joint frequency used to compute frequency to exceed $x / 0$

$c \quad=f$ then joint frequency used to compute annual average $x / 0$

$c$ inorm $=t$ then joint frequency data is normal ized (as in GENII)

$c \quad=f$ then joint frequency data is un-normal ized

$c$ icdf $=t$ then cumulative distribution file created (CDF.OUT)

$c=f$ then no cumulative distribution file created

$c$ ichk $=t$ then $X / Q$ paraneter print option turned on

c $\quad f$ then no paraneter print

$c$ isite $=t$ then $X / Q$ based on joint frequency data for all 16 sectors

$c \quad=f$ then $x / \theta$ based on joint frequency data of individual sectors

$c$ ipop $=t$ then $x / Q$ is population weighted

$c \quad=f$ then no population weight ing

c $X / O$ AND HINO SPEED ADJUSTMENT MODELS:

c ipuff idep isre iwind

$\begin{array}{llll}0 & 0 & 0 & 1\end{array}$

C DIFFUSION COEFFICIENT ADJUSTMENT MODELS:

c iwake ipm iflow ientr
0

C EFFECTIVE RELEASE HEIGHT ADJUSTMENT MODELS:

c (irise igrnd)iwash igrav

$2 \quad 0 \quad 1 \quad 0$

c ipuff = 1 then $X / 0$ calculated using puff model

$c \quad=0$ then $X / 0$ calculated using defaul $t$ cont inuous plune model 


\section{WHC-SD-CP-TI-197 Revision 0}

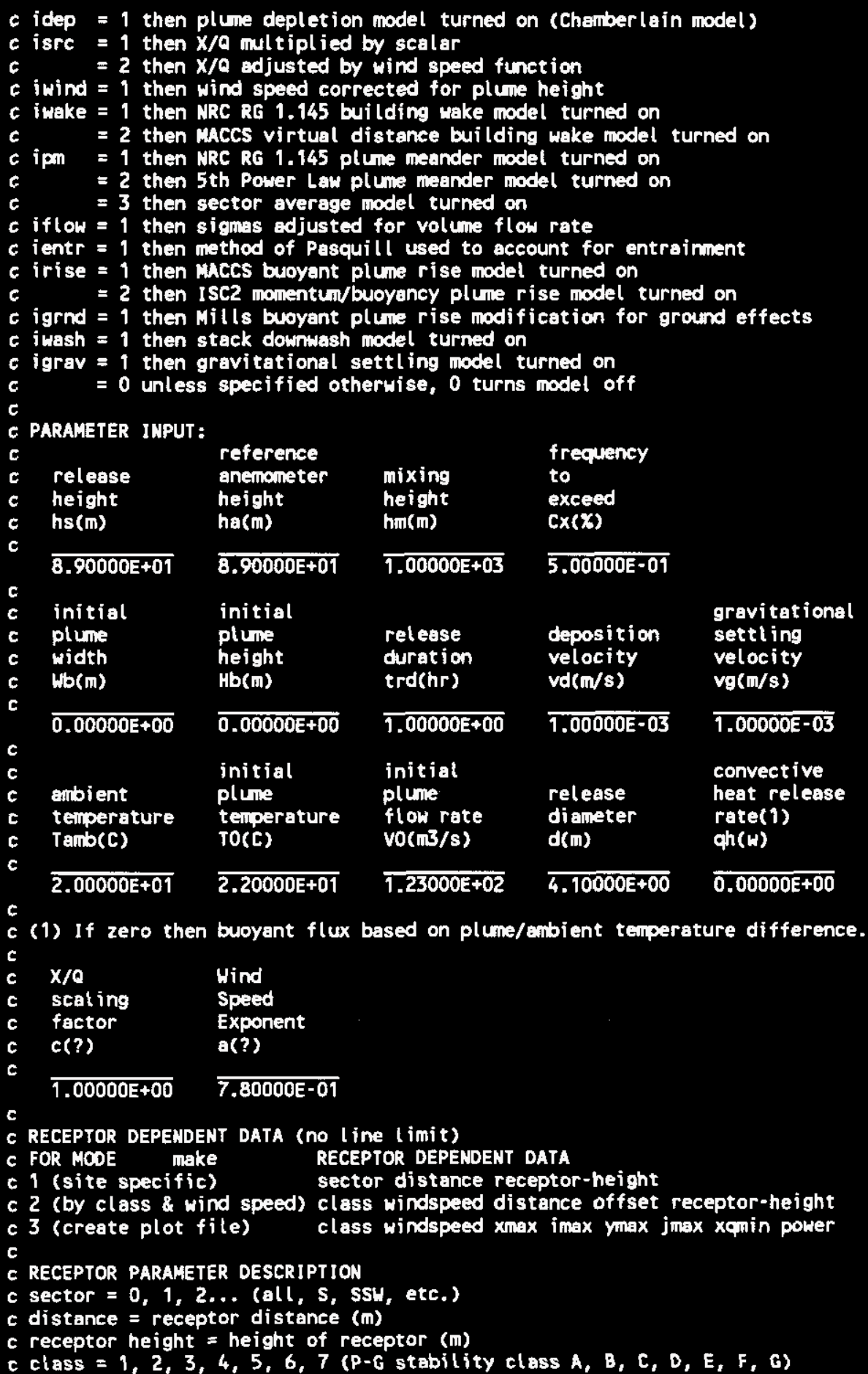




\section{WHC-SD-CP-TI-197 Revision 0}

c windspeed $=$ anemoneter wind speed $(\mathrm{m} / \mathrm{s})$

c offset $=$ of fset from plume centerline (m)

c $x_{\max }=$ maximum distance to plot or calculate to $(\mathrm{m})$

c $i_{\max }=$ distance intervals

c ymax = maximum offset to plot (m)

c $j \max =$ of fset intervals

c xqmin = minimum scaled $x / 0$ to calculate

c power = exponent in power function step size

NOOE:

Site specific $\times / A$ calculated.

LOGICAL CHOICES:

Joint frequency used to calculate annual average $X / Q$.

No normalization of joint frequency.

$X / a$ calculated for single sector.

$x / a$ is population weighted.

MODELS SELECTED:

flow adjustment model.

Sector average model selected.

ISC2 momentum/buoyancy plume model besed on temperature difference.

Stack downwash model selected.

Default Gaussian plume model selected.

Uind velocity corrected for average plune height.

WARNING/ERROR MESSAGES:

UARNING $\# 10$ - $X / Q$ may be underestimated for plume rise scenario without accounting for entraimient.

JOINT FREQUENCY DATA:

200 AREA (HMS) - 89 M - Pasquill A - G (1983 - 1991 Average)

Created 8/26/92 KR

POPULATION DATA:

Population within $80 \mathrm{~km}$ of the Hanford 200 Area, 1990 Census (Beck, et al 1991)

Created 10/21/91 KR, Revised 2/4/92 KR

Population Offsite $99.5 \times$ Chronic stack Level Release

\begin{tabular}{|c|c|c|c|c|c|c|c|c|}
\hline SECTOR & $\begin{array}{l}\text { DISTANCE } \\
\text { (m) }\end{array}$ & $\begin{array}{l}\text { RECEPT } \\
\text { HEIGHT } \\
\text { (m) }\end{array}$ & $\begin{array}{l}\text { SECT. } \\
\text { FREO. } \\
(X)\end{array}$ & POPULATIOW & $\begin{array}{c}\text { TOTAL } \\
\text { POPULATION } \\
\text { SCALED } \\
X / 0 \\
(s / m 3)\end{array}$ & $\begin{array}{c}\text { AVERAGE } \\
\text { IWDIVIDUAL } \\
\text { SCALED } \\
x / 0 \\
\left(\mathrm{~s} / \mathrm{m}^{3}\right)\end{array}$ & $\begin{array}{l}\text { ATM. } \\
\text { STAB. } \\
\text { CLASS }\end{array}$ & $\begin{array}{l}\text { UIND } \\
\text { SPEED } \\
(\mathrm{m} / \mathrm{s})\end{array}$ \\
\hline $\begin{array}{l}\text { S } \\
\text { SSW } \\
\text { SW } \\
\text { WSW } \\
\text { W } \\
\text { WNH } \\
\text { WH } \\
\text { NNH } \\
\text { N } \\
\text { WNE } \\
\text { NE } \\
\text { ENE }\end{array}$ & $\begin{array}{l}80000 \\
80000 \\
80000 \\
80000 \\
80000 \\
80000 \\
80000 \\
80000 \\
80000 \\
80000 \\
80000 \\
80000\end{array}$ & $\begin{array}{l}0 \\
0 \\
0 \\
0 \\
0\end{array}$ & $\begin{array}{l}6.12 \\
4.29 \\
2.82 \\
2.57 \\
4.58 \\
3.81 \\
4.58 \\
4.52 \\
4.33 \\
2.48 \\
3.89 \\
6.27\end{array}$ & $\begin{array}{r}5845 \\
13937 \\
22666 \\
31223 \\
102538 \\
5292 \\
2879 \\
7308 \\
4643 \\
24085 \\
10372 \\
3956\end{array}$ & $\begin{array}{l}2.61 E-05 \\
3.20 E-05 \\
4.61 E-05 \\
4.87 E-05 \\
2.02 E-04 \\
1.22 E-05 \\
8.24 E-06 \\
1.59 E-05 \\
1.07 E-05 \\
2.43 E-05 \\
1.81 E-05 \\
8.95 E-06\end{array}$ & $\begin{array}{l}4.46 E-09 \\
2.30 E-09 \\
2.03 E-09 \\
1.56 E-09 \\
1.97 E-09 \\
2.31 E-09 \\
2.86 E-09 \\
2.18 E-09 \\
2.30 E-09 \\
1.01 E-09 \\
1.75 E-09 \\
2.26 E-09\end{array}$ & & \\
\hline
\end{tabular}


WHC-SD-CP-TI-197 Revision 0

$\begin{array}{llrrrrr}\text { E } & 80000 & 0 & 14.38 & 3992 & 2.18 E-05 & 5.47 E-09 \\ \text { ESE } & 80000 & 0 & 19.41 & 3000 & 1.98 E-05 & 6.60 E-09 \\ \text { SE } & 80000 & 0 & 11.04 & 114734 & 4.55 E-04 & 3.97 E-09 \\ \text { SSE } & 80000 & 0 & 4.73 & 19390 & 5.34 E-05 & 2.75 E-09\end{array}$

